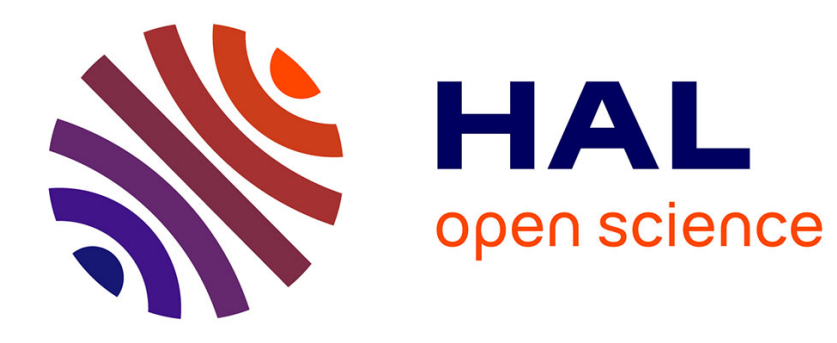

\title{
Volume Viscosity and Internal Energy Relaxation: Error Estimates
}

Vincent Giovangigli, Wen-An Yong

\section{To cite this version:}

Vincent Giovangigli, Wen-An Yong. Volume Viscosity and Internal Energy Relaxation: Error Estimates. 2014. hal-01006275v3

\section{HAL Id: hal-01006275 \\ https://hal.science/hal-01006275v3}

Preprint submitted on 18 Nov 2014

HAL is a multi-disciplinary open access archive for the deposit and dissemination of scientific research documents, whether they are published or not. The documents may come from teaching and research institutions in France or abroad, or from public or private research centers.
L'archive ouverte pluridisciplinaire HAL, est destinée au dépôt et à la diffusion de documents scientifiques de niveau recherche, publiés ou non, émanant des établissements d'enseignement et de recherche français ou étrangers, des laboratoires publics ou privés. 


\title{
Volume Viscosity and Internal Energy Relaxation : Error Estimates
}

\author{
Vincent Giovangigli ${ }^{1}$ and Wen-An Yong ${ }^{2,3}$ \\ ${ }^{1}$ CMAP-CNRS, École Polytechnique, 91128 Palaiseau cedex, FRANCE \\ ${ }^{2}$ ZCAM, Tsinghua University, Beijing, 100084, CHINA \\ ${ }^{3}$ Beijing Computational Science Research Center, Beijing 100084, CHINA
}

\begin{abstract}
We investigate the fast relaxation of internal energy in nonequilibrium gas models derived from the kinetic theory of gases. We establish uniform a priori estimates and existence theorems for symmetric hyperbolic-parabolic systems of partial differential equations with small second order terms and stiff sources. We prove local in time error estimates between the out of equilibrium solution and the one-temperature equilibrium fluid solution for well prepared data and justify the apparition of volume viscosity terms.
\end{abstract}

\section{Introduction}

The kinetic theory of polyatomic gases shows that the volume viscosity coefficient is related to the time required for the internal and translational temperatures to come to equilibrium $[6,16,35,36,3,4,5]$. We establish in this paper local in time error estimates between the solution of an out of equilibrium two-temperature model and the solution of a one-temperature equilibrium model-including volume viscosity terms - when the relaxation time goes to zero.

The system of partial differential equations modeling fluids out of thermodynamic equilibrium as derived from the kinetic theory of gases is first summarized [3, 4]. This system and its symmetrizability properties have been investigated in our previous work [25]. The symmetrizing normal variable w of the out of equilibrium model is taken in the form

$$
\mathrm{w}=\left(\rho, \boldsymbol{v}, \frac{1}{T_{\mathrm{tr}}}-\frac{1}{T_{\mathrm{in}}},-\frac{1}{T}\right)^{t}
$$

where $\rho$ denotes the gas density, $v$ the fluid velocity, $T_{\operatorname{tr}}$ the translational temperature, $T_{\text {in }}$ the internal temperature, and $T$ the local equilibrium temperature. The resulting system of partial differential equations is in the general form

$$
\overline{\mathrm{A}}_{0}(\mathrm{w}) \partial_{t} \mathrm{w}+\sum_{i \in \mathcal{D}} \overline{\mathrm{A}}_{i}(\mathrm{w}) \partial_{i} \mathrm{w}-\epsilon_{d} \sum_{i, j \in \mathcal{D}} \partial_{i}\left(\overline{\mathrm{B}}_{i j}(\mathrm{w}) \partial_{j} \mathrm{w}\right)+\frac{1}{\epsilon} \overline{\mathrm{L}}(\mathrm{w}) \mathrm{w}=\epsilon_{d} \overline{\mathrm{b}}\left(\mathrm{w}, \partial_{\mathrm{x}} \mathrm{w}_{\mathrm{II}}\right),
$$

where $\partial_{t}$ denotes the time derivative operator, $\partial_{i}$ the space derivative operator in the $i$ th direction, $\mathcal{D}=\{1, \ldots, d\}$ the spatial directions, $d$ the space dimension, $\epsilon, \epsilon_{d} \in(0,1]$ two positive parameters and $\mathrm{w}=\left(\mathrm{w}_{\mathrm{I}}, \mathrm{w}_{\mathrm{II}}\right)^{t}$ is decomposed into its hyperbolic components $\mathrm{w}_{\mathrm{I}}$ and parabolic components $\mathrm{w}_{\mathrm{II}}$. The matrix $\overline{\mathrm{A}}_{0}$ is symmetric positive definite and bloc-diagonal, $\overline{\mathrm{A}}_{i}$ are symmetric, $\overline{\mathrm{B}}_{i j}^{t}=\overline{\mathrm{B}}_{j i}, \overline{\mathrm{B}}_{i j}$ have nonzero components only into the right lower $\overline{\mathrm{B}}_{i j}^{\mathrm{II}, \mathrm{II}}$ blocs, $\overline{\mathrm{B}}^{\mathrm{II}, \mathrm{II}}=\sum_{i, j \in \mathcal{D}} \overline{\mathrm{B}}_{i j}^{\mathrm{II}, \mathrm{II}}(\mathrm{w}) \xi_{i} \xi_{j}$ is positive definite for $\boldsymbol{\xi} \in \Sigma^{d-1}, \overline{\mathrm{L}}$ is positive semi-definite with a fixed nullspace $\overline{\mathcal{E}}$, and $\overline{\mathrm{b}}\left(\mathrm{w}, \partial_{\mathrm{x}} \mathrm{w}_{\text {II }}\right)$ is quadratic in the gradients. Denoting by $\pi$ the orthogonal projector onto $\overline{\mathcal{E}}^{\perp}$, the normal variable $w$ is such that we have the commutation relation $\pi \overline{\mathrm{A}}_{0}=\overline{\mathrm{A}}_{0} \pi$. The source term is also naturally in quasilinear form as is typical in a relaxation framework and often encountered in mathematical physics [45]. The small parameter $\epsilon$ is associated with energy relaxation and the small parameter $\epsilon_{d}$ with second order dissipative terms. 
We establish uniform a priori estimates for linearized symmetric hyperbolic-parabolic systems with small dissipation and stiff sources obtained from the nonlinear equations (1.2). Symmetrized forms are important for analyzing hyperbolic as well as hyperbolic-parabolic systems of partial differential equations modeling fluids $[26,17,42,34,29,33,30,31,7,39,20,18,8,9,40,28,15,44,32,19,21,2$, $40,24,9,23]$. A priori estimates are obtained uniformly with respect to the parameters $\epsilon_{d} \in(0,1]$ and $\epsilon \in(0,1]$. The differences with the estimates established by Kawashima [29] are the inclusion of extra terms associated with the fast variable $\pi \mathrm{w} / \epsilon$ and the estimates for time derivatives. Denoting by $\mathrm{w}^{\star} \mathrm{a}$ constant equilibrium state and $\bar{\tau}$ a positive time, we estimate $\mathbf{w}-\mathbf{w}^{\star}$ in the space $C^{0}\left([0, \bar{\tau}], H^{l}\right)$ as well as $\partial_{t} \mathrm{w}$ and $\pi \mathrm{w} / \epsilon$ in $L^{2}\left((0, \bar{\tau}), H^{l-1}\right)$ for $l \geq[d / 2]+2$ where $H^{l}=H^{l}\left(\mathbb{R}^{d}\right)$ denotes the usual Sobolev space when the initial solution is close to the equilibrium manifold. A priori estimates require the commutation between the mass matrix and the orthogonal projector onto the fast manifold $\pi \overline{\mathrm{A}}_{0}=\overline{\mathrm{A}}_{0} \pi$. These estimates lead to local existence theorems for well prepared initial conditions on a time interval independent of both parameters $\epsilon_{d} \in(0,1]$ and $\epsilon \in(0,1]$. Key points for local existence are notably to take into account stiff sources in the linearized equations in order to build approximated solutions, the new estimates for time derivatives, and the convergence rate of successive approximations that may depends on $\epsilon$. Stronger estimates for $\partial_{t} \mathrm{w}$ in $C^{0}\left([0, \bar{\tau}], H^{l-2}\right)$ as well as for $\pi \partial_{t} \mathrm{w} / \epsilon$ in $L^{2}\left((0, \bar{\tau}), H^{l-3}\right)$ with $l \geq[d / 2]+4$ are also established when the initial time derivative is close to the equilibrium manifold. These theorems yield the first existence results for the out of equilibrium two-temperature model derived in [3] and symmetrized in [25]. The situation of ill prepared data is also addressed with inital layers.

We finally investigate the singular limit $\epsilon, \epsilon_{d} \rightarrow 0$ in the system modeling fluids out of thermodynamic equilibrium. Various relaxation models have also been investigated in the literature in different physical and mathematical contexts $[7,8,10,32,37,43,46]$. In order to investigate the asymptotic behavior of solutions as $\epsilon, \epsilon_{d} \rightarrow 0$ we combine a priori estimates out of thermodynamic equilibrium with stability results associated with the equilibrium limit model. The fast variable notably corresponds to the rescaled temperature difference with $\left(T_{\mathrm{tr}}-T_{\mathrm{in}}\right) / \epsilon=-T_{\mathrm{tr}} T_{\mathrm{in}} \pi \mathrm{w} / \epsilon$ and we use that perturbed hyperbolic-parabolic systems with small second order terms and perturbing right hand sides admit local solutions that depend continuously on perturbations. Denoting by $\mathrm{w}_{\mathrm{e}}=\left(\rho_{\mathrm{e}}, \boldsymbol{v}_{\mathrm{e}},-1 / T_{\mathrm{e}}\right)^{t}$ the solution of the equilibrium one-temperature model including the volume viscosity terms and by $\varphi \mathrm{w}=(\rho, \boldsymbol{v},-1 / T)^{t}$ the projection on the slow manifold of the normal variable $\mathbf{w}$ out of equilibrium, we establish that $\varphi \mathrm{w}-\mathrm{w}_{\mathrm{e}}=\mathcal{O}\left(\epsilon\left(\epsilon+\epsilon_{d}\right)\right)$. This justifies the addition of the volume viscosity term $-\kappa_{\mathrm{e}}\left(\boldsymbol{\nabla} \cdot \boldsymbol{v}_{\mathrm{e}}\right) \boldsymbol{I}$ in the viscous tensor $\boldsymbol{\Pi}_{\mathrm{e}}$ at equilibrium

$$
\boldsymbol{\Pi}_{\mathrm{e}}=-\kappa_{\mathrm{e}}\left(\boldsymbol{\nabla} \cdot \boldsymbol{v}_{\mathrm{e}}\right) \boldsymbol{I}-\eta_{\mathrm{e}}\left(\boldsymbol{\nabla} \boldsymbol{v}_{\mathrm{e}}+\left(\boldsymbol{\nabla} \boldsymbol{v}_{\mathrm{e}}\right)^{t}-\frac{2}{3}\left(\boldsymbol{\nabla} \cdot \boldsymbol{v}_{\mathrm{e}}\right) \boldsymbol{I}\right)
$$

where $\kappa_{\mathrm{e}}$ and $\eta_{\mathrm{e}}$ denote the equilibrium volume and shear viscosities, discarding $\mathcal{O}\left(\epsilon\left(\epsilon+\epsilon_{d}\right)\right)$ Burnett type residuals. In the situation where $\epsilon=\epsilon_{d}$, it has also been established that the equilibrium system corresponds to a second order Chapman-Enskog expansion for small relaxation times [25] and the error estimates of $\varphi w-w_{e}$ yields a rigorous jusitification of the second order accuracy. To the author's knowledge, it is the first time that the apparition of volume viscosity terms is justified rigorously with an error estimate. Note incidentally that experimental measurements [38, 41] as well as theoretical calculations $[6,16,35,3]$ have shown that the volume viscosity coefficient is of the same order as the shear viscosity coefficient for polyatomic gases and the impact of volume viscosity in fluid mechanics has also been established $[12,13,11,27,1,3]$.

The nonequilibrium two-temperature model and its symmetrization are summarized in Section 2. A priori estimates and local existence results are established in Section 3. Stability for equilibrium models and convergence of the nonequilibrium model towards the one-temperature model is established in Section 4.

\section{Governing equations}

The system of equations modeling fluids out of thermodynamic equilibrium as derived from the kinetic of gases is summarized and recast into a convenient normal form [3, 4, 25]. The local equilibrium temperature, the volume viscosity coefficient, and the equations at equilibrium are also discussed. 


\subsection{Conservation equations}

In a nonequilibrium gas with internal degrees of freedom, the conservation of mass, momentum, internal energy and total energy may be written in the form [3]

$$
\begin{aligned}
& \partial_{t} \rho+\nabla \cdot(\rho \boldsymbol{v})=0 \\
& \partial_{t}(\rho \boldsymbol{v})+\nabla \cdot(\rho \boldsymbol{v} \otimes \boldsymbol{v}+p \boldsymbol{I})+\boldsymbol{\nabla} \cdot \boldsymbol{\Pi}=0, \\
& \partial_{t}\left(\rho e_{\text {in }}\right)+\nabla \cdot\left(\rho \boldsymbol{v} e_{\text {in }}\right)+\nabla \cdot \boldsymbol{Q}_{\text {in }}=\omega_{\text {in }}, \\
& \partial_{t}\left(\rho\left(e_{\mathrm{tr}}+e_{\mathrm{in}}+\frac{1}{2}|\boldsymbol{v}|^{2}\right)\right)+\boldsymbol{\nabla} \cdot\left(\rho \boldsymbol{v}\left(e_{\mathrm{tr}}+e_{\mathrm{in}}+\frac{1}{2}|\boldsymbol{v}|^{2}\right)+\boldsymbol{v} p\right) \\
& +\boldsymbol{\nabla} \cdot\left(\boldsymbol{Q}_{\mathrm{tr}}+\boldsymbol{Q}_{\mathrm{in}}+\boldsymbol{\Pi} \cdot \boldsymbol{v}\right)=0,
\end{aligned}
$$

where $\boldsymbol{\nabla}$ denotes the space derivative operator, $\rho$ the mass density, $\boldsymbol{v}$ the fluid velocity, $\otimes$ the tensor product symbol, $p$ the pressure, $\boldsymbol{\Pi}$ the viscous tensor, $\boldsymbol{I}$ the unit tensor in the physical space $\mathbb{R}^{d}, e_{\mathrm{in}}$ the internal energy of internal origin per unit mass, $\boldsymbol{Q}_{\text {in }}$ the heat flux of internal origin, $\omega_{\text {in }}$ the energy exchange rate, $e_{\mathrm{tr}}$ the internal energy of translational origin per unit mass, and $\boldsymbol{Q}_{\mathrm{tr}}$ the heat flux of translational origin. The components of $\boldsymbol{v}$ and $\boldsymbol{\nabla}$ are written $\boldsymbol{v}=\left(v_{1}, \ldots, v_{d}\right)^{t}$ and $\boldsymbol{\nabla}=\left(\partial_{1}, \ldots, \partial_{d}\right)^{t}$ where $v_{i}$ denotes the velocity in the $i$ th spatial direction, $\partial_{i}$ the derivation in the $i$ th spatial direction and bold symbols are used for vector or tensor quantities in the physical space $\mathbb{R}^{d}$. The equations (2.2)-(2.4) have to be completed by relations expressing the thermodynamic properties $e_{\mathrm{in}}, e_{\mathrm{tr}}$, and $p$, the rate of energy exchange $\omega_{\mathrm{in}}$, and the transport fluxes $\boldsymbol{\Pi}, \boldsymbol{Q}_{\mathrm{in}}$ and $\boldsymbol{Q}_{\mathrm{tr}}$.

\subsection{Thermodynamics}

The pressure $p$, the total internal energy per unit mass $e$, the internal energy of translational origin per unit mass $e_{\mathrm{tr}}$, and the internal energy of internal origin per unit mass $e_{\mathrm{in}}$ are in the form

$$
p=\rho r T_{\mathrm{tr}}, \quad e=e_{\mathrm{tr}}+e_{\mathrm{in}}, \quad e_{\mathrm{tr}}=c_{\mathrm{v}, \mathrm{tr}} T_{\mathrm{tr}}, \quad e_{\mathrm{in}}=e_{\mathrm{in}, \mathrm{st}}+\int_{T_{\mathrm{st}}}^{T_{\mathrm{in}}} c_{\mathrm{in}}\left(T^{\prime}\right) \mathrm{d} T^{\prime},
$$

where $r$ denotes the gas constant per unit mass, $c_{\mathrm{v}, \mathrm{tr}}=\frac{3}{2} r$ the translational heat at constant volume per unit mass, $T_{\mathrm{tr}}$ the translational temperature, $c_{\mathrm{in}}$ the internal heat per unit mass, $T_{\mathrm{in}}$ the internal temperature, $T_{\mathrm{st}}$ the standard temperature, and $e_{\mathrm{in}, \mathrm{st}}$ the internal formation energy at the standard temperature. We will also use in the following the translational heat at constant pressure per unit mass $c_{p, \operatorname{tr}}=\frac{5}{2} r$ and the formation energy at zero temperature $e_{\mathrm{in}}^{0}=e_{\mathrm{in}}(0)$.

The rate of energy exchange between the translational and internal degrees of freedom $\omega_{\text {in }}$ may also be written [3]

$$
\omega_{\text {in }}=\frac{\rho c_{\text {in }}}{\tau_{\text {in }}}\left(T_{\mathrm{tr}}-T_{\text {in }}\right),
$$

where $\tau_{\text {in }}$ denotes the energy exchange time.

\subsection{Transport fluxes}

In the framework of the kinetic theory of polyatomic gases out of thermodynamic equilibrium, the translational and internal heat fluxes are in the form [3]

$$
\begin{aligned}
& \boldsymbol{Q}_{\mathrm{tr}}=-\lambda_{\mathrm{tr}, \mathrm{tr}} \boldsymbol{\nabla} T_{\mathrm{tr}}-\lambda_{\mathrm{tr}, \mathrm{in}} \boldsymbol{\nabla} T_{\mathrm{in}}, \\
& \boldsymbol{Q}_{\mathrm{in}}=-\lambda_{\mathrm{in}, \mathrm{tr}} \boldsymbol{\nabla} T_{\mathrm{tr}}-\lambda_{\mathrm{in}, \mathrm{in}} \boldsymbol{\nabla} T_{\mathrm{in}},
\end{aligned}
$$

where $\lambda_{\mathrm{tr}, \mathrm{tr}}, \lambda_{\mathrm{tr}, \mathrm{in}}, \lambda_{\mathrm{in}, \mathrm{tr}}$, and $\lambda_{\mathrm{in}, \text { in }}$ denote thermal conductivities. On the other hand, the viscous tensor is given by

$$
\boldsymbol{\Pi}=-\eta\left(\boldsymbol{\nabla} \boldsymbol{v}+(\boldsymbol{\nabla} \boldsymbol{v})^{t}-\frac{2}{d^{\prime}}(\boldsymbol{\nabla} \cdot \boldsymbol{v}) \boldsymbol{I}\right),
$$

where $\eta$ denotes the shear viscosity and $d^{\prime}$ the dimension of the velocity space in the underlying kinetic framework. It will be assumed in the following that the dimension of the kinetic velocity space $d^{\prime}$ is such that $2 \leq d^{\prime}$ and $d \leq d^{\prime}$. The assumption $1 \leq d \leq d^{\prime}$ means that the spatial dimension $d$ of the 
model has eventually been reduced. The assumption $2 \leq d^{\prime}$ is natural since $d^{\prime}=3$ in our physical world and since $\boldsymbol{\Pi}$ is identically zero when $d^{\prime}=1$.

The thermal conductivities $\lambda_{\mathrm{tr}, \mathrm{tr}}, \lambda_{\mathrm{tr}, \mathrm{in}}, \lambda_{\mathrm{in}, \mathrm{tr}}$, and $\lambda_{\mathrm{in} \text {,in }}$ and the shear viscosity $\eta$ are obtained from the kinetic theory of non equilibrium gases [3]. From the expression (2.9) it is also noted that the viscous tensor $\boldsymbol{\Pi}$ does not present a volume viscosity term and our aim is to investigate the apparition of such a contribution in the one-temperature equilibrium limit model as the relaxation time $\tau_{\text {in }}$ goes to zero.

\subsection{Mathematical assumptions}

The mathematical assumptions associated with the thermodynamic properties, the energy exchange rate, and the transport coefficients are the following where $\varkappa \geq 3$ denotes the regularity class $[22,18$, $3,25]$.

$\left(\mathbf{T}_{1}\right) \quad$ The formation energy $e_{\mathrm{in}, \mathrm{st}}$ and formation entropies $s_{\mathrm{tr}, \mathrm{st}}$ and $s_{\mathrm{in}, \mathrm{st}}$ are real constants. The mass per unit mole $m$, the gas constant $R$, and the gas constant per unit mass $r=R / m$ are positive. The internal species heat per unit mass $c_{\mathrm{in}}\left(T_{\mathrm{in}}\right)$ is a $C^{\varkappa-1}$ function over $[0, \infty)$ and there exist constants $\underline{c}$ and $\bar{c}$ such that $0<\underline{c} \leqslant c_{\mathrm{in}}\left(T_{\mathrm{in}}\right) \leqslant \bar{c}$ for all $T_{\mathrm{in}} \geqslant 0$.

$\left(\mathbf{T}_{2}\right) \quad$ The energy exchange rate $\tau_{\mathrm{in}}\left(p, T_{\mathrm{tr}}, T_{\mathrm{in}}\right)$ is in the form

$$
\tau_{\text {in }}=\epsilon \bar{\tau}_{\text {in }}=\epsilon \frac{p^{\mathrm{st}} \bar{\tau}_{\text {in }}^{\mathrm{st}}}{p}
$$

where $\epsilon \in(0,1]$ denotes a positive parameter, $\bar{\tau}_{\mathrm{in}}\left(p, T_{\mathrm{tr}}, T_{\mathrm{in}}\right)=p^{\mathrm{st}} \bar{\tau}_{\mathrm{in}}^{\mathrm{st}} / p$ the rescaled energy exchange time and $\bar{\tau}_{\mathrm{in}}^{\mathrm{st}}\left(T_{\mathrm{tr}}, T_{\mathrm{in}}\right)$ the rescaled energy exchange time at the standard pressure $p^{\mathrm{st}}$ which only depends on $T_{\mathrm{tr}}$ and $T_{\mathrm{in}}$. The rescaled time $\bar{\tau}_{\mathrm{in}}^{\mathrm{st}}$ is a positive $C^{\varkappa}$ function of the two temperatures $T_{\mathrm{tr}}, T_{\mathrm{in}} \in(0, \infty)$.

( $\left.\boldsymbol{T r}_{1}\right) \quad$ The coefficients $\eta, \lambda_{\mathrm{tr}, \mathrm{tr}}, \lambda_{\mathrm{tr}, \mathrm{in}}, \lambda_{\mathrm{in}, \mathrm{tr}}$, and $\lambda_{\mathrm{in}, \mathrm{in}}$ are in the form

$$
\begin{aligned}
\eta=\epsilon_{d} \bar{\eta}, \quad \lambda_{\mathrm{tr}, \mathrm{tr}}= & \epsilon_{d} \bar{\lambda}_{\mathrm{tr}, \mathrm{tr}}, \quad \lambda_{\mathrm{tr}, \mathrm{in}}=\epsilon_{d} \bar{\lambda}_{\mathrm{tr}, \mathrm{in}}, \\
& \lambda_{\mathrm{in}, \mathrm{tr}}=\epsilon_{d} \bar{\lambda}_{\mathrm{in}, \mathrm{tr}}, \quad \lambda_{\mathrm{in}, \text { in }}=\epsilon_{d} \bar{\lambda}_{\mathrm{in}, \mathrm{in}},
\end{aligned}
$$

where $\epsilon_{d} \in(0,1]$ denotes a positive parameter, and $\bar{\eta}, \bar{\lambda}_{\mathrm{tr}, \mathrm{tr}}, \bar{\lambda}_{\mathrm{tr}, \mathrm{in}}, \bar{\lambda}_{\mathrm{in}, \mathrm{tr}}$, and $\bar{\lambda}_{\mathrm{in}, \mathrm{in}}$ the rescaled transport coefficients. The rescaled coefficients $\bar{\eta}, \bar{\lambda}_{\mathrm{tr}, \mathrm{tr}}, \bar{\lambda}_{\mathrm{tr}, \mathrm{in}}, \bar{\lambda}_{\mathrm{in}, \mathrm{tr}}$, and $\bar{\lambda}_{\mathrm{in}, \mathrm{in}}$ are $C^{\varkappa}$ functions of the two temperatures $T_{\mathrm{tr}}, T_{\mathrm{in}} \in(0, \infty)$.

$\left(\operatorname{Tr}_{2}\right) \quad$ For any $T_{\mathrm{tr}}, T_{\mathrm{in}} \in(0, \infty)$, the matrix

$$
\left[\begin{array}{ll}
T_{\mathrm{in}}^{2} \bar{\lambda}_{\mathrm{in}, \mathrm{in}} & T_{\mathrm{tr}}^{2} \bar{\lambda}_{\mathrm{in}, \mathrm{tr}} \\
T_{\mathrm{in}}^{2} \bar{\lambda}_{\mathrm{tr}, \mathrm{in}} & T_{\mathrm{tr}}^{2} \bar{\lambda}_{\mathrm{tr}, \mathrm{tr}}
\end{array}\right],
$$

is symmetric positive definite. In the viscous tensor (2.9), the coefficient $\eta$ is positive and the dimension $d^{\prime}$ of the kinetic velocity space is such that $\max (2, d) \leq d^{\prime}$.

The rescaled energy exchange time $\bar{\tau}_{\text {in }}$ as well as the rescaled transport coefficients $\bar{\eta}, \bar{\lambda}_{\text {tr,tr }}, \bar{\lambda}_{\text {tr,in }}$, $\bar{\lambda}_{\mathrm{in}, \mathrm{tr}}$, and $\bar{\lambda}_{\mathrm{in}, \mathrm{in}}$ have been introduced in order to investigate the fast relaxation limit.

\subsection{Volume viscosity}

The local thermal equilibrium temperature is defined as the unique scalar $T$ such that

$$
e_{\mathrm{tr}}(T)+e_{\mathrm{in}}(T)=e_{\mathrm{tr}}\left(T_{\mathrm{tr}}\right)+e_{\mathrm{in}}\left(T_{\mathrm{in}}\right),
$$

keeping in mind that $e_{\operatorname{tr}}(T)+e_{\text {in }}(T)$ is an increasing function of $T$. The temperature $T$ is a $C^{\varkappa}$ function of $\left(T_{\mathrm{tr}}, T_{\mathrm{in}}\right)$ and is the temperature that would be obtained at local thermal equilibrium $T_{\mathrm{tr}}=T_{\mathrm{in}}$ 
assuming that the internal energy $e_{\mathrm{tr}}+e_{\mathrm{in}}$ is kept fixed. Letting $\widetilde{c}_{\mathrm{in}}=\int_{0}^{1} c_{\mathrm{in}}\left(T_{\mathrm{in}}+s\left(T-T_{\mathrm{in}}\right)\right) \mathrm{d} s$, we may write $e_{\text {in }}(T)-e_{\text {in }}\left(T_{\text {in }}\right)=\left(T-T_{\text {in }}\right) \widetilde{c}_{\text {in }}$ so that $\left(T_{\mathrm{tr}}-T\right) c_{\mathrm{v}, \mathrm{tr}}=\left(T-T_{\mathrm{in}}\right) \widetilde{c}_{\mathrm{in}}$ and $\left(T_{\mathrm{tr}}-T\right) \widetilde{c}_{\mathrm{v}}=\left(T_{\mathrm{tr}}-T_{\mathrm{in}}\right) \widetilde{c}_{\mathrm{in}}$ where $\widetilde{c}_{\mathrm{v}}=c_{\mathrm{v}, \operatorname{tr}}+\widetilde{c}_{\mathrm{in}}\left(T, T_{\mathrm{in}}\right)$. Letting $c_{\mathrm{v}}\left(T_{\mathrm{in}}\right)=c_{\mathrm{v}, \operatorname{tr}}+c_{\mathrm{in}}\left(T_{\mathrm{in}}\right)$ and

$$
\kappa=\kappa\left(T_{\mathrm{tr}}, T_{\mathrm{in}}\right)=\frac{r \widetilde{c}_{\mathrm{in}} p \tau_{\mathrm{in}}}{c_{\mathrm{v}} \widetilde{c}_{\mathrm{v}}}=\epsilon \frac{r \widetilde{c}_{\text {in }} p^{\mathrm{st}} \bar{\tau}_{\text {in }}^{\mathrm{st}}}{c_{\mathrm{v}} \widetilde{c}_{\mathrm{v}}},
$$

the following relation is obtained after some algebra

$$
\begin{aligned}
\rho r\left(T_{\mathrm{tr}}-T\right)=-\kappa \boldsymbol{\nabla} \cdot \boldsymbol{v} & -\frac{\kappa}{p}\left(\boldsymbol{\Pi}: \boldsymbol{\nabla} \boldsymbol{v}+\boldsymbol{\nabla} \cdot \boldsymbol{Q}_{\mathrm{tr}}-\frac{c_{\mathrm{v}, \mathrm{tr}}}{c_{\mathrm{in}}} \boldsymbol{\nabla} \cdot \boldsymbol{Q}_{\mathrm{in}}\right. \\
& \left.+\rho \partial_{t}\left(T_{\mathrm{tr}}-T_{\mathrm{in}}\right)+\rho \boldsymbol{v} \cdot \boldsymbol{\nabla}\left(T_{\mathrm{tr}}-T_{\mathrm{in}}\right)\right) .
\end{aligned}
$$

Note that we have $\kappa=\epsilon \bar{\kappa}$ with $\bar{\kappa}=r \widetilde{c}_{\text {in }} p \bar{\tau}_{\text {in }} /\left(c_{\mathrm{v}} \widetilde{c}_{\mathrm{V}}\right)$ from assumption (2.10). Equation (2.15) is a relaxation equation that yields formally $\rho r\left(T_{\mathrm{tr}}-T\right)=-\kappa \boldsymbol{\nabla} \cdot \boldsymbol{v}+\mathcal{O}\left(\epsilon\left(\epsilon+\epsilon_{d}\right)\right)$ so that both temperatures $T_{\operatorname{tr}}$ and $T_{\mathrm{in}}$ should converge towards the local equilibrium temperature $T$. In the momentum equation, the pressure tensor $\rho r T_{\operatorname{tr}} \boldsymbol{I}+\boldsymbol{\Pi}$ is thus asymptotically in the form

$$
\rho r T_{\mathrm{tr}} \boldsymbol{I}+\boldsymbol{\Pi}=\rho r T \boldsymbol{I}-\kappa(\boldsymbol{\nabla} \cdot \boldsymbol{v}) \boldsymbol{I}-\eta\left(\boldsymbol{\nabla} \boldsymbol{v}+(\boldsymbol{\nabla} \boldsymbol{v})^{t}-\frac{2}{d^{\prime}}(\boldsymbol{\nabla} \cdot \boldsymbol{v}) \boldsymbol{I}\right)+\mathcal{O}\left(\epsilon\left(\epsilon+\epsilon_{d}\right)\right) .
$$

This is in agreement with classical one-temperature models where the pressure $\rho r T$ is evaluated at the thermal equilibrium temperature $T$ and the viscous tensor $\boldsymbol{\Pi}$ includes a volume viscosity term $-\kappa(\boldsymbol{\nabla} \cdot \boldsymbol{v}) \boldsymbol{I}$. Such a physically intuitive derivation may be found in many physics papers and books either in a molecular framework or in a macroscopic fluid framework usually around equilibrium states $[6,16,35,36,3,4,5]$. Numerical simulations using Boltzmann equation have consistently established that the limit one-temperature model is an accurate description of the two temperature fluid when the relaxation time is small [3]. In our previous work [25], it has further been established that the Chapman-Enskog method exactly yields the one-temperature fluid equations with the volume viscosity terms at second order in the fast relaxation limit. The goal of this paper is to justify with an error estimate both the above physically intuitive approximation as well as the accuracy of the two term Chapman-Enskog expansion [25].

\subsection{Quasilinear forms}

Letting $n=d+3$, the conservative variable $\mathbf{u} \in \mathbb{R}^{n}$ associated with equations (2.1)-(2.4) is found to be

$$
\mathbf{u}=\left(\rho, \rho \boldsymbol{v}, \rho e_{\mathrm{in}}, \rho\left(e_{\mathrm{tr}}+e_{\mathrm{in}}+\frac{1}{2}|\boldsymbol{v}|^{2}\right)\right)^{t},
$$

and the natural variable $\mathrm{z} \in \mathbb{R}^{n}$ is defined by $\mathrm{z}=\left(\rho, \boldsymbol{v}, T_{\mathrm{in}}, T_{\mathrm{tr}}\right)^{t}$. For convenience, the velocity components of vectors in $\mathbb{R}^{n}=\mathbb{R} \times \mathbb{R}^{d} \times \mathbb{R}^{2}$ are generally written as vectors of $\mathbb{R}^{d}$. We introduce the corresponding open sets $\mathcal{O}_{\mathrm{u}}$ and $\mathcal{O}_{\mathrm{z}}$ of $\mathbb{R}^{n}$ given by

$$
\mathcal{O}_{\mathrm{u}}=\left\{\mathrm{u}=\left(\mathrm{u}_{\rho}, \mathrm{u}_{\boldsymbol{v}}, \mathrm{u}_{\mathrm{in}}, \mathrm{u}_{\mathrm{tl}}\right)^{t} \in \mathbb{R}^{n} ; \mathrm{u}_{\rho}>0, \mathrm{u}_{\mathrm{in}}>\mathrm{u}_{\rho} e_{\mathrm{in}}^{0}, \mathrm{u}_{\mathrm{tl}}>f\left(\mathrm{u}_{\rho}, \mathrm{u}_{\boldsymbol{v}}, \mathrm{u}_{\mathrm{in}}\right)\right\},
$$

where $f\left(\mathrm{u}_{\rho}, \mathrm{u}_{\boldsymbol{v}}, \mathrm{u}_{\text {in }}\right)=\mathrm{u}_{\text {in }}+\frac{1}{2} \mathrm{u}_{\boldsymbol{v}} \cdot \mathrm{u}_{\boldsymbol{v}} / \mathrm{u}_{\rho}$ and $\mathcal{O}_{\mathrm{z}}=(0, \infty) \times \mathbb{R}^{d} \times(0, \infty)^{2}$. The following proposition has been established in our previous work [25].

Proposition 2.1. Assuming that $\left(\mathrm{T}_{1}\right)$ holds, the map $\mathrm{z} \longmapsto \mathrm{u}$ is a $C^{\varkappa}$ diffeomorphism from the open set $\mathcal{O}_{\mathrm{z}}$ onto the open set $\mathcal{O}_{\mathrm{u}}$ and the open set $\mathcal{O}_{\mathrm{u}}$ is convex.

The equations modeling fluids out of thermodynamic equilibrium may then be written in the compact form

$$
\partial_{t} \mathrm{u}+\sum_{i \in \mathcal{D}} \partial_{i} \mathrm{~F}_{i}+\epsilon_{d} \sum_{i \in \mathcal{D}} \partial_{i} \mathrm{~F}_{i}^{\text {diss }}-\frac{1}{\epsilon} \Omega=0
$$

where $\mathrm{F}_{i}$ denotes the convective flux in the $i$ th direction, $\epsilon_{d}$ the Knudsen number, $\mathrm{F}_{i}^{\text {diss }}$ the rescaled dissipative flux in the $i$ th direction, $\epsilon$ the relaxation parameter, and $\Omega$ the rescaled source term. 
From the governing equations (2.1)-(2.4) the convective flux $F_{i}$ in the $i$ th direction is given by

$$
\mathrm{F}_{i}=\left(\rho v_{i}, \rho \boldsymbol{v} v_{i}+p \boldsymbol{e}_{i}, \rho e_{\mathrm{in}} v_{i},\left(\rho e_{\mathrm{tr}}+\rho e_{\mathrm{in}}+\frac{1}{2} \rho|\boldsymbol{v}|^{2}+p\right) v_{i}\right)^{t},
$$

where $\boldsymbol{e}_{i}$ denotes the basis vectors of $\mathbb{R}^{d}$. Similarly, the dissipative flux $\epsilon_{d} \mathrm{~F}_{i}^{\text {diss }}$ is given by

$$
\epsilon_{d} \mathrm{~F}_{i}^{\mathrm{diss}}=\left(0, \boldsymbol{\Pi}_{i}, Q_{\mathrm{in}, i}, Q_{\mathrm{tr}, i}+Q_{\mathrm{in}, i}+\boldsymbol{\Pi}_{i} \cdot \boldsymbol{v}\right)^{t}
$$

where $\boldsymbol{Q}_{\mathrm{tr}}=\left(Q_{\mathrm{tr}, 1}, \ldots, Q_{\mathrm{tr}, d}\right)^{t}, \boldsymbol{Q}_{\mathrm{in}}=\left(Q_{\mathrm{in}, 1}, \ldots, Q_{\mathrm{in}, d}\right)^{t}, \Pi_{i j}, 1 \leq i, j \leq d$, are the components of the viscous tensor $\boldsymbol{\Pi}$, and $\boldsymbol{\Pi}_{i}=\left(\Pi_{1 i}, \ldots, \Pi_{d i}\right)^{t}$. The source term is finally given by

$$
\frac{1}{\epsilon} \Omega=\left(0, \mathbf{0}, \omega_{\mathrm{in}}, 0\right)^{t}
$$

From the expressions of the viscous tensor and of the heat fluxes we deduce that the dissipative fluxes $\mathrm{F}_{i}^{\text {diss }}$ are linear expression in terms of spatial derivatives of $\mathrm{z}$ and may thus be written in the form $\mathrm{F}_{i}^{\text {diss }}=-\sum_{j \in \mathcal{D}} \widehat{B}_{i j}(\mathrm{z}) \partial_{j} \mathrm{z}$. Using Proposition 2.1, we may then write that $\mathrm{F}_{i}^{\text {diss }}=-\sum_{j \in \mathcal{D}} \mathrm{B}_{i j}(\mathrm{u}) \partial_{j} \mathrm{u}$ where the dissipation matrix $\mathrm{B}_{i j}$ is defined as $\mathrm{B}_{i j}=\widehat{\mathrm{B}}_{i j} \partial_{\mathrm{u}} \mathrm{z}$. Further introducing the Jacobian matrices of the convective fluxes $A_{i}=\partial_{\mathrm{u}} \mathrm{F}_{i}$ the governing equations are finally rewritten into the compact form

$$
\partial_{t} \mathrm{u}+\sum_{i \in \mathcal{D}} \mathrm{A}_{i}(\mathrm{u}) \partial_{i} \mathrm{u}-\epsilon_{d} \sum_{i, j \in \mathcal{D}} \partial_{i}\left(\mathrm{~B}_{i j}(\mathrm{u}) \partial_{j} \mathrm{u}\right)-\frac{1}{\epsilon} \Omega(\mathrm{u})=0 .
$$

In our previous work [25], all possible normal variables leading to a symmetric hyperbolic-parabolic structure have been shown to be in the form $\left(\mathcal{F}_{\mathrm{I}}(\rho), \mathcal{F}_{\mathrm{II}}\left(\boldsymbol{v}, T_{\mathrm{in}}, T_{\mathrm{tr}}\right)\right)^{t}$ where $\mathcal{F}_{\mathrm{I}}$ and $\mathcal{F}_{\text {II }}$ are diffeomorphisms in $\mathbb{R}$ and $\mathbb{R}^{d+2}$, respectively. The natural variable $\mathbf{z}$ is in particular a normal variable but for convenience the following normal variable will be used

$$
\mathrm{w}=\left(\rho, \boldsymbol{v}, \frac{1}{T_{\mathrm{tr}}}-\frac{1}{T_{\mathrm{in}}},-\frac{1}{T}\right)^{t}
$$

The density $\mathrm{w}_{\mathrm{I}}=\rho$ is the hyperbolic variable, $\mathrm{w}_{\mathrm{II}}=\left(\boldsymbol{v}, \frac{1}{T_{\mathrm{tr}}}-\frac{1}{T_{\mathrm{in}}},-\frac{1}{T}\right)^{t}$ the parabolic variable, and the corresponding normal form has been evaluated [25]. The third component of w goes to zero with the relaxation time and the other components $(\rho, \boldsymbol{v},-1 / T)^{t}$ are expected to converge towards the corresponding normal variable at thermodynamic equilibrium $\mathrm{w}_{\mathrm{e}}=\left(\rho_{\mathrm{e}}, \boldsymbol{v}_{\mathrm{e}},-1 / T_{\mathrm{e}}\right)^{t}$.

Theorem 2.2. Assume that $\left(\mathrm{T}_{1}\right)\left(\mathrm{T}_{2}\right)$ and $\left(\mathrm{Tr}_{1}\right)\left(\mathrm{Tr}_{2}\right)$ hold. Then the map $\mathrm{u} \rightarrow \mathrm{w}$ is a $C^{\varkappa-1}$ diffeomorphism from the open set $\mathcal{O}_{\mathbf{u}}$ onto the open set $\mathcal{O}_{\mathbf{w}}=(0, \infty) \times \mathbb{R}^{d} \times \mathbb{R} \times(-\infty, 0)$. The system written in the $\mathrm{w}$ variable is in the normal form with a source term in quasilinear form

$$
\overline{\mathrm{A}}_{0}(\mathrm{w}) \partial_{t} \mathrm{w}+\sum_{i \in \mathcal{D}} \overline{\mathrm{A}}_{i}(\mathrm{w}) \partial_{i} \mathrm{w}-\epsilon_{d} \sum_{i, j \in \mathcal{D}} \partial_{i}\left(\overline{\mathrm{B}}_{i j}(\mathrm{w}) \partial_{j} \mathrm{w}\right)+\frac{1}{\epsilon} \overline{\mathrm{L}}(\mathrm{w}) \mathrm{w}=\epsilon_{d} \overline{\mathrm{b}}\left(\mathrm{w}, \partial_{\mathrm{x}} \mathrm{w}_{\mathrm{II}}\right),
$$

and the matrices $\overline{\mathrm{A}}_{0}, \overline{\mathrm{A}}_{i}, i \in \mathcal{D}, \overline{\mathrm{B}}_{i j}, i, j \in \mathcal{D}, \overline{\mathrm{L}}$, as well as the quadratic residual $\overline{\mathrm{b}}$ and the source term $\bar{\Omega}$ are detailed in previous work [25]. The matrix $\overline{\mathrm{A}}_{0}$ is symmetric positive definite, $\overline{\mathrm{A}}_{i}, i \in \mathcal{D}$, are symmetric, we have $\overline{\mathrm{B}}_{i j}^{t}=\overline{\mathrm{B}}_{j i}, i, j \in \mathcal{D}, \overline{\mathrm{L}}$ is positive semi-definite with a fixed nullspace $\overline{\mathcal{E}}$, and $\overline{\mathrm{b}}\left(\mathrm{w}, \partial_{\mathrm{x}} \mathrm{w}_{\mathrm{II}}\right)$ is quadratic in the gradients. Using the bloc structure induced by the partitioning between hyperbolic and parabolic variable, $\overline{\mathrm{A}}_{0}$ is bloc diagonal, $\overline{\mathrm{B}}_{i j}$ has nonzero coefficients only in the right lower bloc $\overline{\mathrm{B}}_{i j}^{\mathrm{II}, \mathrm{II}}$ and for any $\boldsymbol{\xi}$ in the sphere $\Sigma^{d-1}$ the matrix $\overline{\mathrm{B}}^{\mathrm{II}, \mathrm{II}}(\mathrm{w}, \boldsymbol{\xi})=\sum_{i, j \in \mathcal{D}} \overline{\mathrm{B}}_{i j}(\mathrm{w}) \xi_{i} \xi_{j}$ is positive definite. The matrices $\overline{\mathrm{B}}_{i j}$ have the structure $\overline{\mathrm{B}}_{i j}=\frac{1}{r} \overline{\mathrm{B}}^{\lambda} \delta_{i j}+\frac{\bar{\eta}}{r T_{\mathrm{tr}}} \overline{\mathrm{B}}_{i j}^{\eta}$ where $\overline{\mathrm{B}}^{\lambda}$ is associated with thermal conductivities and $\overline{\mathrm{B}}_{i j}^{\eta}$ with shear viscous effects. The equilibrium linear manifold with respect to the normal variable is the fixed subspace $\overline{\mathcal{E}}=\mathbb{R} \times \mathbb{R}^{d} \times\{0\} \times \mathbb{R}$ and the normal variable $\mathrm{w}$ is quasilinear on the fast manifold $\overline{\mathcal{E}}^{\perp}=\mathbb{R e}_{d+2}$. Finally, the normal variable is compatible with the fast manifold so that $\pi \overline{\mathrm{A}}_{0}=\overline{\mathrm{A}}_{0} \pi$. 


\subsection{Equations at equilibrium}

In order to investigate the fast relaxation limit $\epsilon \rightarrow 0$ we will need to establish a stability theorem for the equations governing fluids at thermodynamic equilibrium that are summarized in this section. The equations modeling one-temperature fluids are in the form $[6,16,18]$

$$
\begin{aligned}
& \partial_{t} \rho_{\mathrm{e}}+\nabla \cdot\left(\rho_{\mathrm{e}} \boldsymbol{v}_{\mathrm{e}}\right)=0, \\
& \partial_{t}\left(\rho_{\mathrm{e}} \boldsymbol{v}_{\mathrm{e}}\right)+\boldsymbol{\nabla} \cdot\left(\rho_{\mathrm{e}} \boldsymbol{v}_{\mathrm{e}} \otimes \boldsymbol{v}_{\mathrm{e}}+p_{\mathrm{e}} \boldsymbol{I}\right)+\boldsymbol{\nabla} \cdot \boldsymbol{\Pi}_{\mathrm{e}}=0, \\
& \partial_{t}\left(\rho e_{\mathrm{e}}+\frac{1}{2} \rho\left|\boldsymbol{v}_{\mathrm{e}}\right|^{2}\right)+\boldsymbol{\nabla} \cdot\left(\boldsymbol{v}_{\mathrm{e}}\left(\rho e_{\mathrm{e}}+\frac{1}{2} \rho\left|\boldsymbol{v}_{\mathrm{e}}\right|^{2}+p_{\mathrm{e}}\right)\right)+\boldsymbol{\nabla} \cdot\left(\boldsymbol{Q}_{\mathrm{e}}+\boldsymbol{\Pi}_{\mathrm{e}} \cdot \boldsymbol{v}_{\mathrm{e}}\right)=0,
\end{aligned}
$$

where the subscript e denotes thermodynamic equilibrium, $\rho_{\mathrm{e}}$ the mass density, $\boldsymbol{v}_{\mathrm{e}}$ the fluid velocity, $p_{\mathrm{e}}$ the pressure, $\boldsymbol{\Pi}_{\mathrm{e}}$ the viscous tensor involving the volume viscosity, $e_{\mathrm{e}}$ the internal energy per unit mass, and $\boldsymbol{Q}_{\mathrm{e}}$ the heat flux.

The pressure $p_{\mathrm{e}}$ and the internal energy per unit mass $e_{\mathrm{e}}$ are in the form $p_{\mathrm{e}}=\rho_{\mathrm{e}} r T_{\mathrm{e}}$ and $e_{\mathrm{e}}=$ $e_{\mathrm{e}, \mathrm{st}}+\int_{T_{\mathrm{st}}}^{T_{\mathrm{e}}} c_{\mathrm{V}}\left(T^{\prime}\right) \mathrm{d} T^{\prime}$ where $c_{\mathrm{v}}\left(T_{\mathrm{e}}\right)=c_{\mathrm{v}, \mathrm{tr}}+c_{\mathrm{in}}\left(T_{\mathrm{e}}\right)$ denotes the heat at constant volume per unit mass, $T_{\mathrm{e}}$ the equilibrium temperature, $e_{\mathrm{e}, \mathrm{st}}$ formation energy at the standard temperature and we have $e_{\mathrm{e}}\left(T_{\mathrm{e}}\right)=e_{\mathrm{tr}}\left(T_{\mathrm{e}}\right)+e_{\mathrm{in}}\left(T_{\mathrm{e}}\right)$. The equilibrium viscous tensor is in the form

$$
\boldsymbol{\Pi}_{\mathrm{e}}=-\kappa_{\mathrm{e}}\left(T_{\mathrm{e}}\right)\left(\boldsymbol{\nabla} \cdot \boldsymbol{v}_{\mathrm{e}}\right) \boldsymbol{I}-\eta_{\mathrm{e}}\left(T_{\mathrm{e}}\right)\left(\boldsymbol{\nabla} \boldsymbol{v}_{\mathrm{e}}+\left(\boldsymbol{\nabla} \boldsymbol{v}_{\mathrm{e}}\right)^{t}-\frac{2}{d^{\prime}}\left(\boldsymbol{\nabla} \cdot \boldsymbol{v}_{\mathrm{e}}\right) \boldsymbol{I}\right)
$$

where $\eta_{\mathrm{e}}\left(T_{\mathrm{e}}\right)=\eta\left(T_{\mathrm{e}}, T_{\mathrm{e}}\right)$ and $\kappa_{\mathrm{e}}\left(T_{\mathrm{e}}\right)=\kappa\left(T_{\mathrm{e}}, T_{\mathrm{e}}\right)$ so that $\kappa_{\mathrm{e}}=r c_{\text {in }} p \tau_{\text {in }} / c_{\mathrm{v}}^{2}$, and the heat flux is given by $\boldsymbol{Q}_{\mathrm{e}}=-\lambda_{\mathrm{e}}\left(T_{\mathrm{e}}\right) \boldsymbol{\nabla} T_{\mathrm{e}}$, with $\lambda_{\mathrm{e}}\left(T_{\mathrm{e}}\right)=\lambda_{\text {tr, tr }}\left(T_{\mathrm{e}}, T_{\mathrm{e}}\right)+\lambda_{\text {tr,in }}\left(T_{\mathrm{e}}, T_{\mathrm{e}}\right)+\lambda_{\text {in,tr }}\left(T_{\mathrm{e}}, T_{\mathrm{e}}\right)+\lambda_{\text {in,in }}\left(T_{\mathrm{e}}, T_{\mathrm{e}}\right)$.

Letting $n_{\mathrm{e}}=d+2$, the conservative variable $\mathrm{u}_{\mathrm{e}} \in \mathbb{R}^{n_{\mathrm{e}}}$ associated with equations (2.23)-(2.25) is $\mathrm{u}_{\mathrm{e}}=\left(\rho_{\mathrm{e}}, \rho_{\mathrm{e}} \boldsymbol{v}_{\mathrm{e}}, \rho e_{\mathrm{e}}+\frac{1}{2} \rho_{\mathrm{e}} \boldsymbol{v}_{\mathrm{e}} \cdot \boldsymbol{v}_{\mathrm{e}}\right)^{t}$ and the corresponding natural variable reads $\mathrm{z}_{\mathrm{e}}=\left(\rho_{\mathrm{e}}, \boldsymbol{v}_{\mathrm{e}}, T_{\mathrm{e}}\right)^{t}$. The corresponding open sets are given by $\mathcal{O}_{\mathrm{u}_{\mathrm{e}}}=\left\{\mathrm{u}_{\mathrm{e}}=\left(\mathrm{u}_{\rho}, \mathrm{u}_{\boldsymbol{v}}, \mathrm{u}_{\mathrm{tl}}\right)^{t} \in \mathbb{R}^{n_{\mathrm{e}}} ; \mathrm{u}_{\rho}>0, \mathrm{u}_{\mathrm{tl}}>f^{\mathrm{e}}\left(\mathrm{u}_{\rho}, \mathrm{u}_{\boldsymbol{v}}\right\}\right.$ where $f^{\mathrm{e}}\left(\mathrm{u}_{\rho}, \mathrm{u}_{\boldsymbol{v}}\right)=\mathrm{u}_{\rho} e_{\mathrm{e}}^{0}+\frac{1}{2} \frac{\mathrm{u}_{\boldsymbol{v}} \cdot \mathrm{u}_{\boldsymbol{v}}}{\mathrm{u}_{\rho}}$ and $\mathcal{O}_{\mathrm{z}_{\mathrm{e}}}=(0, \infty) \times \mathbb{R}^{d} \times(0, \infty)$. The map $\mathrm{z}_{\mathrm{e}} \rightarrow \mathrm{u}_{\mathrm{e}}$ is easily shown to be a $C^{\varkappa}$ diffeomorphism from $\mathcal{O}_{z_{\mathrm{e}}}$ onto $\mathcal{O}_{\mathrm{u}_{\mathrm{e}}}$. Introducing the convective and dissipative fluxes of the equilibrium fluid model (2.23)-(2.25)

$$
\begin{gathered}
\mathrm{F}_{i}^{\mathrm{e}}=\left(\rho_{\mathrm{e}} v_{\mathrm{e} i}, \rho_{\mathrm{e}} \boldsymbol{v}_{\mathrm{e}} v_{\mathrm{e} i}+p_{\mathrm{e}} \boldsymbol{e}_{i},\left(\rho e_{\mathrm{e}}+p_{\mathrm{e}}+\frac{1}{2} \rho_{\mathrm{e}}\left|\boldsymbol{v}_{\mathrm{e}}\right|^{2}\right) v_{\mathrm{e} i}\right)^{t}, \\
\epsilon_{d} \mathrm{~F}_{i}^{\mathrm{e} \operatorname{diss}}=\left(0, \boldsymbol{\Pi}_{\mathrm{e} i}, Q_{\mathrm{e} i}+\boldsymbol{\Pi}_{\mathrm{e} i} \cdot \boldsymbol{v}_{\mathrm{e}}\right)^{t}
\end{gathered}
$$

using straighforward notation, the system at equilibrium may be rewritten in qualilinear form

$$
\partial_{t} \mathrm{u}_{\mathrm{e}}+\sum_{i \in \mathcal{D}} \mathrm{A}_{i}^{\mathrm{e}}\left(\mathrm{u}_{\mathrm{e}}\right) \partial_{i} \mathrm{u}_{\mathrm{e}}-\epsilon_{d} \sum_{i, j \in \mathcal{D}} \partial_{i}\left(\mathrm{~B}_{i j}^{\mathrm{e}}\left(\mathrm{u}_{\mathrm{e}}\right) \partial_{j} \mathrm{u}_{\mathrm{e}}\right)=0
$$

where $\mathrm{A}_{i}^{\mathrm{e}}, i \in \mathcal{D}$, denote the Jacobian matrices $\mathrm{A}_{i}^{\mathrm{e}}=\partial_{\mathrm{u}_{\mathrm{e}}} \mathrm{F}_{i}^{\mathrm{e}}$ and $\mathrm{B}_{i j}^{\mathrm{e}}, i, j \in \mathcal{D}$, the dissipation matrices at equilibrium with $\mathrm{F}_{i}^{\mathrm{e} d i s s}=-\sum_{j \in \mathcal{D}} \mathrm{B}_{i j}^{\mathrm{e}} \partial_{j} \mathrm{u}_{\mathrm{e}}[29,31,18]$. The equations of the one-temperature equilibrium model may also be written in normal form [25] with the normal variable

$$
\mathrm{w}_{\mathrm{e}}=\left(\rho_{\mathrm{e}}, \boldsymbol{v}_{\mathrm{e}},-\frac{1}{T_{\mathrm{e}}}\right)^{t}
$$

where the density $\mathrm{w}_{\mathrm{eI}}=\rho_{\mathrm{e}}$ is the hyperbolic variable and $\mathrm{w}_{\mathrm{eII}}=\left(\boldsymbol{v}_{\mathrm{e}},-\frac{1}{T_{\mathrm{e}}}\right)^{t}$ the parabolic variable.

Theorem 2.3. Assume that $\left(\mathrm{T}_{1}\right)\left(\mathrm{T}_{2}\right)$ and $\left(\operatorname{Tr}_{1}\right)\left(\mathrm{Tr}_{2}\right)$ hold. Then the map $\mathrm{v}_{\mathrm{e}} \rightarrow \mathrm{w}_{\mathrm{e}}$ is a $C^{\varkappa-1}$ diffeomorphism from the open set $\mathcal{O}_{v_{\mathrm{e}}}$ onto the open set $\mathcal{O}_{w_{\mathrm{e}}}=(0, \infty) \times \mathbb{R}^{d} \times(-\infty, 0)$. The system written in the $\mathrm{w}_{\mathrm{e}}$ variable is of the normal form

$$
\overline{\mathrm{A}}_{0}^{\mathrm{e}}\left(\mathrm{w}_{\mathrm{e}}\right) \partial_{t} \mathrm{w}_{\mathrm{e}}+\sum_{i \in \mathcal{D}} \overline{\mathrm{A}}_{i}^{\mathrm{e}}\left(\mathrm{w}_{\mathrm{e}}\right) \partial_{i} \mathrm{w}_{\mathrm{e}}-\epsilon_{d} \sum_{i, j \in \mathcal{D}} \partial_{i}\left(\overline{\mathrm{B}}_{i j}^{\mathrm{e}}\left(\mathrm{w}_{\mathrm{e}}\right) \partial_{j} \mathrm{w}_{\mathrm{e}}\right)=\epsilon_{d} \overline{\mathrm{b}}_{\mathrm{e}}\left(\mathrm{w}_{\mathrm{e}}, \partial_{\mathrm{x}} \mathrm{w}_{\text {IIe }}\right),
$$

where $\overline{\mathrm{A}}_{0}^{\mathrm{e}}, \overline{\mathrm{A}}_{i}^{\mathrm{e}}, i \in \mathcal{D}, \overline{\mathrm{B}}_{i j}^{\mathrm{e}}$, are detailed in [25] and have regularity at least $\varkappa-1$, and $\overline{\mathrm{b}}_{\mathrm{e}}$ is a quadratic residual. The matrices at equilibirum are related to the analog matrices out of equilibrium with the 
relations $\overline{\mathrm{A}}_{0}^{\mathrm{e}}=\psi^{t} \overline{\mathrm{A}}_{0}\left(\psi \mathrm{w}_{\mathrm{e}}\right) \psi, \overline{\mathrm{A}}_{i}^{\mathrm{e}}=\psi^{t} \overline{\mathrm{A}}_{i}\left(\psi \mathrm{w}_{\mathrm{e}}\right) \psi$ with

$$
\psi=\left[\begin{array}{ccc}
1 & 0_{1, d} & 0 \\
0_{d, 1} & \boldsymbol{I} & 0_{d, 1} \\
0 & 0_{1, d} & 0 \\
0 & 0_{1, d} & 1
\end{array}\right] .
$$

Moreover, the dissipation matrices $\overline{\mathrm{B}}_{i j}^{\mathrm{e}}$ have the structure $\overline{\mathrm{B}}_{i j}^{\mathrm{e}}=\frac{1}{r} \overline{\mathrm{B}}^{\lambda, \mathrm{e}} \delta_{i j}+\frac{\bar{\kappa}_{\mathrm{e}}}{r T_{\mathrm{e}}} \overline{\mathrm{B}}_{i j}^{\kappa, \mathrm{e}}+\frac{\bar{\eta}_{\mathrm{e}}}{r T_{\mathrm{e}}} \overline{\mathrm{B}}_{i j}^{\eta, \mathrm{e}}$ with $\overline{\mathrm{B}}^{\lambda, \mathrm{e}}=\psi^{t} \overline{\mathrm{B}}^{\lambda}\left(\psi \mathrm{w}_{\mathrm{e}}\right) \psi$ and $\overline{\mathrm{B}}_{i j}^{\eta, \mathrm{e}}=\psi^{t} \overline{\mathrm{B}}_{i j}^{\eta}\left(\psi \mathrm{w}_{\mathrm{e}}\right) \psi$ whereas the matrices $\overline{\mathrm{B}}_{i j}^{\kappa, \mathrm{e}}, i, j \in \mathcal{D}$, are given in [25].

Denoting by $\varphi$ the linear operator $\varphi=\psi^{t}$ where $\psi$ is the rectangular matrix (2.32), one of the goal of this paper is to establish that the equilibrium projection $\varphi w$ of the normal variable $w$ out of thermodynamic equilibrium is close to the normal variable $w_{e}$ at thermodynamic equilibrium so that $\varphi \mathrm{w}-\mathrm{w}_{\mathrm{e}}=\mathcal{O}\left(\epsilon\left(\epsilon+\epsilon_{d}\right)\right)$.

\section{$3 \quad$ Hyperbolic-parabolic systems with stiff source terms}

We investigate in this section local existence theorems for hyperbolic-parabolic systems of partial differential equations in normal form with small second order terms and stiff sources. We generally follow the elegant formalism and methods of proof of Kawashima [29], the differences being due to the stiff sources.

\subsection{Preliminaries}

We consider an abstract hyperbolic-parabolic system with small second order terms and stiff sources in normal form. The system is written

$$
\overline{\mathrm{A}}_{0}(\mathrm{w}) \partial_{t} \mathrm{w}+\sum_{i \in \mathcal{D}} \overline{\mathrm{A}}_{i}(\mathrm{w}) \partial_{i} \mathrm{w}-\epsilon_{d} \sum_{i, j \in \mathcal{D}} \partial_{i}\left(\overline{\mathrm{B}}_{i j}(\mathrm{w}) \partial_{j} \mathrm{w}\right)+\frac{1}{\epsilon} \overline{\mathrm{L}}(\mathrm{w}) \mathrm{w}=\epsilon_{d} \overline{\mathrm{b}}\left(\mathrm{w}, \partial_{\times} \mathrm{w}_{\mathrm{II}}\right),
$$

where $\mathrm{w}=\left(\mathrm{w}_{\mathrm{I}}, \mathrm{w}_{\text {II }}\right)^{t} \in \mathcal{O}_{\mathrm{w}}, \mathcal{O}_{\mathrm{w}}$ is an open set of $\mathbb{R}^{n}, \mathrm{w}_{\mathrm{I}}$ are the hyperbolic components, $\mathrm{w}_{\mathrm{II}}$ the parabolic components, and $\epsilon_{d}, \epsilon \in(0,1]$ are two positive parameters. The dimensions of the hyperbolic and parabolic components are denoted by $n_{\mathrm{I}}$ and $n_{\mathrm{II}}$ respectively so that $n=n_{\mathrm{I}}+n_{\mathrm{II}}$. The matrices $\overline{\mathrm{A}}_{0}, \overline{\mathrm{A}}_{i}, \overline{\mathrm{B}}_{i j}$, and $\overline{\mathrm{L}}$ are assumed to have at least regularity $\varkappa-2$. We will generally assume that $\varkappa$ is as large as required by the various theorems in the following, in particular that $\varkappa-3 \geq l+1 \geq l_{0}+2$ where $l_{0}=[d / 2]+1$. The matrix $\overline{\mathrm{A}}_{0}$ is symmetric positive definite, the matrices $\overline{\mathrm{A}}_{i}$ are symmetric, $\overline{\mathrm{B}}_{i j}$ satisfy $\overline{\mathrm{B}}_{i j}^{t}=\overline{\mathrm{B}}_{j i}$, and $\overline{\mathrm{L}}$ is positive semi-definite with a fixed nullspace $\overline{\mathcal{E}}$. The matrices $\overline{\mathrm{A}}_{0}$ and $\overline{\mathrm{B}}_{i j}$, $i, j \in \mathcal{D}$, have the bloc structure

$$
\overline{\mathrm{A}}_{0}=\left[\begin{array}{cc}
\overline{\mathrm{A}}_{0}^{\mathrm{I}, \mathrm{I}} & 0_{n_{\mathrm{I}}, n_{\mathrm{II}}} \\
0_{n_{\mathrm{II}}, n_{\mathrm{I}}} & \overline{\mathrm{A}}_{0}^{\mathrm{II}, \mathrm{II}}
\end{array}\right], \quad \overline{\mathrm{B}}_{i j}=\left[\begin{array}{cc}
0_{n_{\mathrm{I}}, n_{\mathrm{I}}} & 0_{n_{\mathrm{I}}, n_{\mathrm{II}}} \\
0_{n_{\mathrm{II}}, n_{\mathrm{I}}} & \overline{\mathrm{B}}_{i j}^{\mathrm{IIIIII}}
\end{array}\right],
$$

and $\overline{\mathrm{B}}^{\mathrm{II}, \mathrm{II}}(\mathrm{w}, \boldsymbol{\xi})=\sum_{i, j \in \mathcal{D}} \overline{\mathrm{B}}_{i j}^{\mathrm{II}, \mathrm{II}}(\mathrm{w}) \xi_{i} \xi_{j}$ is positive definite for $\mathrm{w} \in \mathcal{O}_{\mathrm{w}}$ and $\boldsymbol{\xi} \in \Sigma^{d-1}$. The quadratic source term is also in the form

$$
\overline{\mathrm{b}}\left(\mathrm{w}, \partial_{\mathrm{x}} \mathrm{w}_{\mathrm{II}}\right)=\sum_{i, j \in \mathcal{D}} \overline{\mathrm{M}}_{i j}(\mathrm{w}) \partial_{i} \mathrm{w} \partial_{j} \mathrm{w}=\left(0, \sum_{i, j \in \mathcal{D}} \overline{\mathrm{M}}_{i j}^{\mathrm{II}, \mathrm{II}, \mathrm{II}}(\mathrm{w}) \partial_{i} \mathrm{w}_{\mathrm{II}} \partial_{j} \mathrm{w}_{\mathrm{II}},\right)^{t}
$$

where $\overline{\mathrm{M}}_{i j}$ are third order tensors depending on $\mathrm{w}$ with at least regularity $\varkappa-3$. Denoting by $\pi$ the orthogonal projector onto the orthogonal of the equilibrium manifold $\overline{\mathcal{E}}^{\perp}$, we assume that $\overline{\mathrm{A}}_{0}$ satisfies the compatibility condition

$$
\pi \overline{\mathrm{A}}_{0}(\mathrm{w})=\overline{\mathrm{A}}_{0}(\mathrm{w}) \pi, \quad \mathrm{w} \in \mathcal{O}_{\mathrm{w}} .
$$


We are only interested in well prepared initial data in this section, that is, we assume that the initial condition $\mathrm{w}_{0}$ is close to the equilibrium manifold $\overline{\mathcal{E}}$ in such a way that $\pi \mathrm{w}_{0}$ is small. The situation of ill prepared data will be investigated with initial layers.

We denote by $\mathbf{u}^{\star}$ and $\mathbf{w}^{\star}$ corresponding constant equilibrium states in the $\mathrm{u}$, and $\mathrm{w}$ variables respectively, so that $w^{\star} \in \mathcal{O}_{w} \cap \overline{\mathcal{E}}$ and $\pi w^{\star}=0$. We denote by $|\bullet|_{l}$ the norm in the Sobolev space $H^{l}=H^{l}\left(\mathbb{R}^{d}\right)$ and otherwise $\|\bullet\|_{A}$ in the functional space $A$. If $\alpha=\left(\alpha_{1}, \ldots, \alpha_{d}\right) \in \mathbb{N}^{d}$ is a multiindex, we denote as usual by $\partial^{\alpha}$ the differential operator $\partial_{1}^{\alpha_{1}} \cdots \partial_{d}^{\alpha_{d}}$ and by $|\alpha|$ its order $|\alpha|=\alpha_{1}+\cdots+\alpha_{d}$. The square of $k^{\text {th }}$ derivatives of a scalar function $\phi$, like $T, \rho$, or $v_{i}, 1 \leq i \leq d$, is defined by

$$
\left|\partial^{k} \phi\right|^{2}=\sum_{|\alpha|=k} \frac{k !}{\alpha !}\left(\partial^{\alpha} \phi\right)^{2}=\sum_{1 \leq i_{1}, \ldots, i_{k} \leq d}\left(\partial_{i_{1}} \cdots \partial_{i_{k}} \phi\right)^{2},
$$

where $k ! / \alpha$ ! are the multinomial coefficients and similarly, for a vector function like $\boldsymbol{v}$ we define $\left|\partial^{k} \boldsymbol{v}\right|^{2}=$ $\sum_{1 \leq i \leq d}\left|\partial^{k} v_{i}\right|^{2}$. Finally, for any map $\phi:[0, \bar{\tau}] \times \mathbb{R}^{d} \rightarrow \mathbb{R}^{n}$ where $\bar{\tau}>0$ is positive and for any $\tau \in[0, \bar{\tau}]$, we denote by $\phi(\tau)$ the partial map $\mathrm{x} \rightarrow \phi(\tau, \mathrm{x})$ defined over $\mathbb{R}^{d}$.

\subsection{A priori estimates}

We consider in this section linearized equations in the form

$$
\overline{\mathrm{A}}_{0}(\mathrm{w}) \partial_{t} \widetilde{\mathrm{w}}+\sum_{i \in \mathcal{D}} \overline{\mathrm{A}}_{i}(\mathrm{w}) \partial_{i} \widetilde{\mathrm{w}}-\epsilon_{d} \sum_{i, j \in \mathcal{D}} \overline{\mathrm{B}}_{i j}(\mathrm{w}) \partial_{i} \partial_{j} \widetilde{\mathrm{w}}+\frac{1}{\epsilon} \overline{\mathrm{L}}(\mathrm{w}) \widetilde{\mathrm{w}}=\mathrm{f}+\epsilon_{d} \mathrm{~g}
$$

Such linearized equations (3.5) are useful in order to build sequences of successive approximations that converge towards solutions of the nonlinear equations (3.1) as well as to estimate the derivatives of such solutions. For a given $\bar{\tau}>0$ and $l \geq l_{0}+1$ where $l_{0}=[d / 2]+1$, we assume that $\mathrm{w}$ is such that

$$
\begin{aligned}
& \left\{\begin{array}{l}
\mathrm{w}_{\mathrm{I}}-\mathrm{w}_{\mathrm{I}}^{\star} \in \mathcal{C}^{0}\left([0, \bar{\tau}], H^{l}\right) \cap \mathcal{C}^{1}\left([0, \bar{\tau}], H^{l-1}\right), \\
\mathrm{w}_{\mathrm{II}}-\mathrm{w}_{\mathrm{II}}^{\star} \in \mathcal{C}^{0}\left([0, \bar{\tau}], H^{l}\right) \cap \mathcal{C}^{1}\left([0, \bar{\tau}], H^{l-2}\right),
\end{array}\right. \\
& \partial_{t} \mathrm{w}_{\mathrm{II}} \in L^{2}\left((0, \bar{\tau}), H^{l-1}\right),
\end{aligned}
$$

and we define

$$
\sup _{0 \leq \tau \leq \bar{\tau}}\left|\mathrm{w}(\tau)-\mathrm{w}^{\star}\right|_{l}^{2}=M^{2}, \quad \int_{0}^{\bar{\tau}}\left|\partial_{t} \mathrm{w}(\tau)\right|_{l-1}^{2} d \tau=M_{1}^{2} .
$$

We consider $\mathcal{O}_{0}$ such that $\overline{\mathcal{O}}_{0} \subset \mathcal{O}_{\mathrm{w}}, d_{1}$ such that $0<d_{1}<d\left(\overline{\mathcal{O}}_{0}, \partial \mathcal{O}_{\mathrm{w}}\right)$, and define

$$
\mathcal{O}_{1}=\left\{\mathrm{w} \in \mathcal{O}_{\mathrm{w}} ; d\left(\mathrm{w}, \overline{\mathcal{O}}_{0}\right)<d_{1}\right\} .
$$

It is also assumed that $w_{0}$ and $w$ are such that

$$
\mathrm{w}_{0}(\mathrm{x})=\mathrm{w}(0, \mathrm{x}) \in \mathcal{O}_{0}, \quad \mathrm{w}(t, \mathrm{x}) \in \mathcal{O}_{1}, \quad t \in[0, \bar{\tau}], \quad \mathrm{x} \in \mathbb{R}^{d} .
$$

The following priori estimates for linearized equations will be of fundamental importance for existence theorem of the full quasilinear system (3.1). When the source terms are not stiff such estimates have been established by Kawashima [29]. The estimates in the situation of stiff sources differ by the inclusion of new terms associated with the fast variable $\pi \mathrm{w} / \epsilon$ as well as for the time derivatives which cannot anymore be estimated directly from the governing equations but require well chosen test functions. In particular, the time derivatives are not in the space $\mathcal{C}^{0}\left([0, \bar{\tau}], H^{l-2}\right)$ uniformly in $\epsilon$ but only in the space $L^{2}\left([0, \bar{\tau}], H^{l-2}\right)$. Stronger estimates in $\mathcal{C}^{0}\left([0, \bar{\tau}], H^{l-2}\right)$, obtained in the next section, indeed require the boundedness of $\left|\pi \partial_{t} \mathrm{w}_{0}\right|_{l-3}^{2} / \epsilon$.

Theorem 3.1. Let $l \geq l_{0}+1$ with $l_{0}=[d / 2]+1$, consider the linearized system (3.5), and assume that the solution $\widetilde{\mathrm{w}}$ is such that

$$
\begin{aligned}
& \widetilde{\mathrm{W}}_{\mathrm{I}}-\widetilde{\mathrm{W}}_{\mathrm{I}}^{\star} \in \mathcal{C}^{0}\left([0, \bar{\tau}], H^{l}\right) \cap \mathcal{C}^{1}\left([0, \bar{\tau}], H^{l-1}\right), \\
& \widetilde{\mathrm{W}}_{\mathrm{II}}-\widetilde{\mathrm{w}}_{\mathrm{II}}^{\star} \in \mathcal{C}^{0}\left([0, \bar{\tau}], H^{l}\right) \cap \mathcal{C}^{1}\left([0, \bar{\tau}], H^{l-2}\right) \cap L^{2}\left((0, \bar{\tau}), H^{l+1}\right),
\end{aligned}
$$


where $\widetilde{\mathrm{W}}^{\star}=\left(\widetilde{\mathrm{W}}_{\mathrm{I}}^{\star}, \widetilde{\mathrm{W}}_{\mathrm{II}}^{\star}\right)^{t}$ is a constant state $\widetilde{\mathrm{w}}^{\star} \in \overline{\mathcal{E}}$. Further assume that

$$
\begin{gathered}
\mathrm{f} \in C^{0}\left([0, \bar{\tau}], H^{l-1}\right) \cap L^{1}\left([0, \bar{\tau}], H^{l}\right), \\
\mathrm{g} \in C^{0}\left([0, \bar{\tau}], H^{l-1}\right), \quad \mathrm{g}_{\mathrm{I}}=0,
\end{gathered}
$$

and denote by $\widetilde{\mathrm{w}}_{0}$ the initial state $\widetilde{\mathrm{w}}_{0}(\mathrm{x})=\widetilde{\mathrm{w}}(0, \mathrm{x})$. Then there exists constants $\mathrm{c}_{1}\left(\mathcal{O}_{1}\right) \geq 1$ and $\mathrm{c}_{2}\left(\mathcal{O}_{1}, M\right) \geq 1$, with $\mathrm{c}_{2}\left(\mathcal{O}_{1}, M\right)$ increasing with $M$, such that for any $t \in[0, \bar{\tau}]$

$$
\begin{gathered}
\sup _{0 \leq \tau \leq t}\left\{\left|\widetilde{\mathrm{w}}(\tau)-\widetilde{\mathrm{w}}^{\star}\right|_{l}^{2}+\frac{1}{\epsilon}|\pi \widetilde{\mathrm{w}}(\tau)|_{0}^{2}\right\}+\epsilon_{d} \int_{0}^{t}\left|\widetilde{\mathrm{w}}_{\mathrm{II}}(\tau)-\widetilde{\mathrm{w}}_{\mathrm{II}}^{\star}\right|_{l+1}^{2} d \tau \\
+\frac{1}{\epsilon} \int_{0}^{t}|\pi \widetilde{\mathrm{w}}(\tau)|_{l}^{2} d \tau \leq \mathrm{c}_{1}^{2} \exp \left(\mathrm{c}_{2}\left(t+M_{1} \sqrt{t}\right)\right)\left(\left|\widetilde{\mathrm{w}}_{0}-\widetilde{\mathrm{w}}^{\star}\right|_{l}^{2}+\frac{1}{\epsilon}\left|\pi \widetilde{\mathrm{w}}_{0}\right|_{0}^{2}\right. \\
\left.+\epsilon_{d} \mathrm{c}_{2} \int_{0}^{t}\left|\mathrm{~g}_{\mathrm{II}}(\tau)\right|_{l-1}^{2} d \tau+\mathrm{c}_{2}\left\{\int_{0}^{t}|\mathrm{f}(\tau)|_{l} d \tau\right\}^{2}+\mathrm{c}_{2} \int_{0}^{t}|\pi \mathrm{f}(\tau)|_{0}^{2} d \tau\right), \\
\frac{1}{\epsilon} \sup _{0 \leq \tau \leq t}|\pi \widetilde{\mathrm{w}}(\tau)|_{l-1}^{2}+\frac{1}{\epsilon^{2}} \int_{0}^{t}|\pi \widetilde{\mathrm{w}}(\tau)|_{l-1}^{2} d \tau+\int_{0}^{t}\left|\partial_{t} \widetilde{\mathrm{w}}(\tau)\right|_{l-1}^{2} d \tau \\
\leq \mathrm{c}_{2} \exp \left(\mathrm{c}_{2}\left(t+M_{1} \sqrt{t}\right)\right)\left(\left|\widetilde{\mathrm{w}}_{0}-\widetilde{\mathrm{w}}^{\star}\right|_{l}^{2}+\frac{1}{\epsilon}\left|\pi \widetilde{\mathrm{w}}_{0}\right|_{l-1}^{2}+\epsilon_{d} \int_{0}^{t}\left|\mathrm{~g}_{\mathrm{II}}\right|_{l-1}^{2} d \tau\right. \\
\left.+\left\{\int_{0}^{t}|\mathrm{f}|_{l} d \tau\right\}^{2}+\int_{0}^{t}|\mathrm{f}|_{l-1}^{2} d \tau\right) .
\end{gathered}
$$

Proof. The lengthy proof is given in Appendix A.

\subsection{Local existence}

We first restate an existence theorem for the linearized equations (3.5) which is a coupled system of hyperbolic-parabolic type established by Kawashima [29]. These linearized coupled hyperbolicparabolic solutions are then used in order to establish the existence of local solutions for the full nonlinear system (3.1).

Proposition 3.2. Let $l \geq l_{0}+1$ where $l_{0}=[d / 2]+1, \bar{\tau}>0$, assume that $\mathrm{w}$ is such that (3.6), (3.7) and (3.10) hold, that $\mathrm{f}$ and $\mathrm{g}$ satisfy (3.12) and (3.13), and that $\widetilde{\mathrm{w}}_{0}$ is such that $\widetilde{\mathrm{w}}_{0}-\widetilde{\mathrm{w}}^{\star} \in H^{l}$ for some constant state $\widetilde{\mathrm{w}}^{\star} \in \overline{\mathcal{E}}$. Then there exists a unique solution $\widetilde{\mathrm{w}}$ to the linearized equations (3.5) with initial condition $\widetilde{\mathrm{w}}_{0}$ and regularity (3.11).

We now establish a local existence theorem on a time interval $\bar{\tau}>0$ independent of $\epsilon_{d}$ and $\epsilon$ for the system of partial differential equation in normal form (3.1). Such an existence theorem is a fundamental step toward a convergence theorem for $\epsilon, \epsilon_{d} \rightarrow 0$. Since we are interested in convergence results on time intervals including the time origin $t=0$, we assume in this section that the initial data is well prepared or equivalently that $\pi \mathrm{w}_{0}$ is small. We follow the elegant method of proof of Kawashima in his seminal work on hyperbolic-parabolic systems [29]. The differences are in the definition of the approximated solutions which include the stiff sources, in the definition of the invariant set by iteration, and fundamentally in the convergence rate of the successive approximated solutions which may indeed depend on $\epsilon$.

Theorem 3.3. Let $d \geq 1$ and $l \geq l_{0}+1$, be integers with $l_{0}=[d / 2]+1$, and let $b>0$ be given. Let $\mathcal{O}_{0}$ such that $\overline{\mathcal{O}}_{0} \subset \mathcal{O}_{\mathrm{w}}, d_{1}$ such that $0<d_{1}<d\left(\overline{\mathcal{O}}_{0}, \partial \mathcal{O}_{\mathrm{w}}\right)$, and define $\mathcal{O}_{1}=\left\{\mathrm{w} \in \mathcal{O}_{\mathrm{w}} ; d\left(\mathrm{w}, \overline{\mathcal{O}}_{0}\right)<d_{1}\right\}$. There exists $\bar{\tau}>0$ depending on $\mathcal{O}_{1}$ and $b$, and independent on $\epsilon_{d} \in(0,1]$ and $\epsilon \in(0,1]$, such that for any $\mathrm{w}_{0}$ with

$$
\left|\mathrm{w}_{0}-\mathrm{w}^{\star}\right|_{l}^{2}+\frac{1}{\epsilon}\left|\pi \mathrm{w}_{0}\right|_{l-1}^{2}<b^{2}
$$

and $\mathrm{w}_{0} \in \overline{\mathcal{O}}_{0}$, there exists a unique local solution $\mathrm{w}$ to the system

$$
\overline{\mathrm{A}}_{0}(\mathrm{w}) \partial_{t} \mathrm{w}+\sum_{i \in \mathcal{D}} \overline{\mathrm{A}}_{i}(\mathrm{w}) \partial_{i} \mathrm{w}-\epsilon_{d} \sum_{i, j \in \mathcal{D}} \partial_{i}\left(\overline{\mathrm{B}}_{i j}(\mathrm{w}) \partial_{j} \mathrm{w}\right)+\frac{1}{\epsilon} \overline{\mathrm{L}}(\mathrm{w}) \mathrm{w}=\epsilon_{d} \overline{\mathrm{b}}\left(\mathrm{w}, \partial_{\mathrm{x}} \mathrm{w}_{\mathrm{II}}\right),
$$


with initial condition

$$
\mathrm{w}(0, \mathrm{x})=\mathrm{w}_{0}(\mathrm{x}), \quad \mathrm{x} \in \mathbb{R}^{d}
$$

such that

$$
\mathrm{w}(t, \mathrm{x}) \in \mathcal{O}_{1}, \quad t \in[0, \bar{\tau}], \quad \mathrm{x} \in \mathbb{R}^{d}
$$

and

$$
\begin{aligned}
& \mathrm{w}_{\mathrm{I}}-\mathrm{w}_{\mathrm{I}}^{\star} \in \mathcal{C}^{0}\left([0, \bar{\tau}], H^{l}\right) \cap \mathcal{C}^{1}\left([0, \bar{\tau}], H^{l-1}\right), \\
& \mathrm{w}_{\mathrm{II}}-\mathrm{w}_{\mathrm{II}}^{\star} \in \mathcal{C}^{0}\left([0, \bar{\tau}], H^{l}\right) \cap \mathcal{C}^{1}\left([0, \bar{\tau}], H^{l-2}\right) \cap L^{2}\left((0, \bar{\tau}), H^{l+1}\right) .
\end{aligned}
$$

In addition, there exists $C>0$ which only depend on $\mathcal{O}_{1}$ and $b$, such that

$$
\begin{aligned}
& \sup _{0 \leq \tau \leq \bar{\tau}}\left(\left|\mathrm{w}(\tau)-\mathrm{w}^{\star}\right|_{l}^{2}+\frac{1}{\epsilon}|\pi \mathrm{w}(\tau)|_{l-1}^{2}\right)+\epsilon_{d} \int_{0}^{\bar{\tau}}\left|\mathrm{w}_{\mathrm{II}}(\tau)-\mathrm{w}_{\mathrm{II}}^{\star}\right|_{l+1}^{2} d \tau \\
& +\frac{1}{\epsilon} \int_{0}^{\bar{\tau}}|\pi \mathrm{w}(\tau)|_{l}^{2} d \tau+\frac{1}{\epsilon^{2}} \int_{0}^{\bar{\tau}}|\pi \mathrm{w}(\tau)|_{l-1}^{2} d \tau+\int_{0}^{\bar{\tau}}\left|\partial_{t} \mathrm{w}(\tau)\right|_{l-1}^{2} d \tau \\
& \leq C\left(\left|\mathrm{w}_{0}-\mathrm{w}^{\star}\right|_{l}^{2}+\frac{1}{\epsilon}\left|\pi \mathrm{w}_{0}\right|_{l-1}^{2}\right) .
\end{aligned}
$$

Proof. Solutions to the nonlinear system (3.17) are fixed points $\widetilde{w}=\mathrm{w}$ of the linearized equations [29]

$$
\overline{\mathrm{A}}_{0}(\mathrm{w}) \partial_{t} \widetilde{\mathrm{w}}+\sum_{i \in \mathcal{D}} \overline{\mathrm{A}}_{i}(\mathrm{w}) \partial_{i} \widetilde{\mathrm{w}}-\epsilon_{d} \sum_{i, j \in \mathcal{D}} \overline{\mathrm{B}}_{i j}(\mathrm{w}) \partial_{i} \partial_{j} \widetilde{\mathrm{w}}+\frac{1}{\epsilon} \overline{\mathrm{L}}(\mathrm{w}) \widetilde{\mathrm{w}}=\epsilon_{d} \mathrm{~g}
$$

with

$$
\mathrm{g}\left(\mathrm{w}, \partial_{\mathrm{x}} \mathrm{w}\right)=\sum_{i, j \in \mathcal{D}} \partial_{i}\left(\overline{\mathrm{B}}_{i j}(\mathrm{w})\right) \partial_{j} \mathrm{w}-\sum_{i, j \in \mathcal{D}} \partial_{i}\left(\partial_{\mathrm{w}} \mathrm{v}\right)^{t}\left(\partial_{\mathrm{v}} \mathrm{w}\right)^{t} \overline{\mathrm{B}}_{i j} \partial_{j} \mathrm{w}
$$

Fixed points are investigated in the space $\mathrm{w} \in \mathrm{X}_{\bar{\tau}}^{l}\left(\mathcal{O}_{1}, M, M_{1}\right)$ defined by $\mathrm{w}_{\mathrm{I}}-\mathrm{w}_{\mathrm{I}}^{\star} \in \mathcal{C}^{0}\left([0, \bar{\tau}], H^{l}\right), \partial_{t} \mathrm{w}_{\mathrm{I}} \in$ $\mathcal{C}^{0}\left([0, \bar{\tau}], H^{l-1}\right), \mathrm{w}_{\mathrm{II}}-\mathrm{w}_{\mathrm{II}}^{\star} \in \mathcal{C}^{0}\left([0, \bar{\tau}], H^{l}\right) \cap L^{2}\left((0, \bar{\tau}), H^{l+1}\right), \partial_{t} \mathrm{w}_{\mathrm{II}} \in \mathcal{C}^{0}\left([0, \bar{\tau}], H^{l-2}\right) \cap L^{2}\left((0, \bar{\tau}), H^{l-1}\right)$, $\mathrm{w}(t, \mathrm{x}) \in \mathcal{O}_{1}$, and

$$
\begin{aligned}
& \sup _{0 \leq \tau \leq \bar{\tau}}\left|\mathrm{w}(\tau)-\mathrm{w}^{\star}\right|_{l}^{2}+\epsilon_{d} \int_{0}^{\bar{\tau}}\left|\mathrm{w}_{\mathrm{II}}(\tau)-\mathrm{w}_{\mathrm{II}}^{\star}\right|_{l+1}^{2} d \tau+\frac{1}{\epsilon} \int_{0}^{\bar{\tau}}|\pi \mathrm{w}(\tau)|_{l}^{2} d \tau \leq M^{2}, \\
& \frac{1}{\epsilon} \sup _{0 \leq \tau \leq \bar{\tau}}|\pi \mathrm{w}(\tau)|_{l-1}^{2}+\frac{1}{\epsilon^{2}} \int_{0}^{\bar{\tau}}|\pi \mathrm{w}(\tau)|_{l-1}^{2} d \tau+\int_{0}^{\bar{\tau}}\left|\partial_{t} \mathrm{w}(\tau)\right|_{l-1}^{2} d \tau \leq M_{1}^{2}
\end{aligned}
$$

For $\mathrm{w}$ in $\mathrm{X}_{\bar{\tau}}^{l}\left(\mathcal{O}_{1}, M, M_{1}\right)$, we may use the estimates established for linearized systems in Theorem 3.1 of Section 3.2. Noting also that $\mathrm{f}=0, \mathrm{~g}_{\mathrm{I}}=0$, and that $\mathrm{g}_{\mathrm{II}}$ is quadratic in the gradients, we obtain upper bounds in the form

$$
|\mathrm{g}(t)|_{l-1}^{2} \leq \mathrm{c}_{2} M^{2}, \quad t \in[0, \bar{\tau}]
$$

and the constants $c_{2}$ of this estimate may be taken identical to the constant of the linear estimates, upon taking the maximum of both constants. Using assumption (3.16) and combining these bounds with the linear estimates (3.14) and (3.15), we obtain that

$$
\begin{aligned}
& \sup _{0 \leq \tau \leq t}\left|\widetilde{\mathrm{w}}(\tau)-\mathrm{w}^{\star}(\tau)\right|_{l}^{2}+\epsilon_{d} \int_{0}^{t}\left|\widetilde{\mathrm{w}}_{\mathrm{II}}(\tau)-\mathrm{w}_{\mathrm{II}}^{\star}\right|_{l+1}^{2} d \tau \\
& +\frac{1}{\epsilon} \int_{0}^{t}|\pi \widetilde{\mathrm{w}}(\tau)|_{l}^{2} d \tau \leq \mathrm{c}_{1}^{2} \exp \left(\mathrm{c}_{2}\left(t+M_{1} \sqrt{t}\right)\right)\left(b^{2}+t \epsilon_{d} \mathrm{c}_{2}^{2} M^{2}\right) \\
& \frac{1}{\epsilon} \sup _{0 \leq \tau \leq t}|\pi \widetilde{\mathrm{w}}(\tau)|_{l-1}^{2}+\frac{1}{\epsilon^{2}} \int_{0}^{t}|\pi \widetilde{\mathrm{w}}(\tau)|_{l-1}^{2} d \tau+\int_{0}^{t}\left|\partial_{t} \widetilde{\mathrm{w}}(\tau)\right|_{l-1}^{2} d \tau \leq \\
& \mathrm{c}_{2} \exp \left(\mathrm{c}_{2}\left(t+M_{1} \sqrt{t}\right)\right)\left(b^{2}+t \epsilon_{d} \mathrm{c}_{2} M^{2}\right) .
\end{aligned}
$$


We now define

$$
M_{b}=2 \mathrm{c}_{1}\left(\mathcal{O}_{1}\right) b, \quad M_{1 b}=\mathrm{c}_{2}\left(\mathcal{O}_{1}, M_{b}\right) 2 \mathrm{c}_{1}\left(\mathcal{O}_{1}\right) b .
$$

Let then be $\bar{\tau} \leq 1$ small enough such that

$$
\begin{gathered}
\exp \left(\mathrm{c}_{2}\left(\mathcal{O}_{1}, M_{b}\right)\left(\bar{\tau}+M_{1 b} \sqrt{\bar{\tau}}\right)\right) \leq 2, \\
\mathrm{c}_{2}^{2}\left(\mathcal{O}_{1}, M_{b}\right) \bar{\tau}\left(2 \mathrm{c}_{1}\left(\mathcal{O}_{1}\right)\right)^{2} \leq 1, \\
\mathrm{c}_{0} M_{1 b} \sqrt{\bar{\tau}}<d_{1}
\end{gathered}
$$

where we have used $\|\phi\|_{L^{\infty}} \leq \mathrm{c}_{0}|\phi|_{l-1}$. Then for any $\mathrm{w} \in \mathrm{X}_{\bar{\tau}}^{l}\left(\mathcal{O}_{1}, M_{b}, M_{1 b}\right)$, any $\mathrm{w}_{0}(\mathrm{x})$ such that $\mathrm{w}_{0}-\mathrm{w}^{\star} \in H^{l}, \mathrm{w}_{0} \in \overline{\mathcal{O}}_{0}$, and $\left|\mathrm{w}_{0}-\mathrm{w}^{\star}\right|_{l}^{2}+\left|\pi \mathrm{w}_{0}\right|_{l-1}^{2} / \epsilon<b^{2}$, and any $\epsilon_{d}, \epsilon \in(0,1]$, the solution $\widetilde{\mathrm{w}}$ to the linearized equations with initial condition $\mathrm{w}_{0}$ stays in the space $\mathrm{X}_{\bar{\tau}}^{l}\left(\mathcal{O}_{1}, M_{b}, M_{1 b}\right)$. More specifically, letting $\widetilde{M}^{2}$ and $\widetilde{M}_{1}^{2}$ be the maximum of the left hand sides of (3.22) and (3.23) respectively, we obtain from (3.22) that

$$
\widetilde{M}^{2} \leq 2 \mathrm{c}_{1}^{2} b^{2}\left(1+4 \epsilon_{d} \mathrm{c}_{1}^{2} \mathrm{c}_{2}^{2} \bar{\tau}\right) \leq 4 \mathrm{c}_{1}^{2} b^{2}=M_{b}^{2}
$$

and from (3.23) we deduce that

$$
\widetilde{M}_{1}^{2} \leq 2 c_{2}^{2} b^{2}\left(1+4 \epsilon_{d} \bar{\tau} c_{1}^{2}\right) \leq M_{1 b}^{2},
$$

since $4 \bar{\tau} c_{1}^{2} \leq 4 \bar{\tau} c_{1}^{2} c_{2}^{2} \leq 1$ and finally that $\left\|\widetilde{\mathrm{w}}-\mathrm{w}^{\star}\right\|_{L^{\infty}} \leq \mathrm{c}_{0} M_{1 \alpha} \sqrt{\bar{\tau}}<d_{1}$ and we have established that the space $\mathrm{X}_{\bar{\tau}}^{l}\left(\mathcal{O}_{1}, M_{b}, M_{1 b}\right)$ is stable.

Let $\mathrm{w}$ and $\widehat{\mathrm{w}}$ be in $\mathrm{X}_{\bar{\tau}}^{l}\left(\mathcal{O}_{1}, M_{b}, M_{1 b}\right)$, let $\mathrm{w}_{0}(\mathrm{x})$ and $\widehat{\mathrm{w}}_{0}(\mathrm{x})$ such that $\mathrm{w}_{0}-\mathrm{w}^{\star} \in H^{l}, \widehat{\mathrm{w}}_{0}-\mathrm{w}^{\star} \in H^{l}$, $\mathrm{w}_{0}, \widehat{\mathrm{w}}_{0} \in \overline{\mathcal{O}}_{0},\left|\mathrm{w}_{0}-\mathrm{w}^{\star}\right|_{l}^{2}+\left|\pi \mathrm{w}_{0}\right|_{l-1}^{2} / \epsilon<b^{2},\left|\widehat{\mathrm{w}}_{0}-\mathrm{w}^{\star}\right|_{l}^{2}+\left|\pi \widehat{\mathrm{w}}_{0}\right|_{l-1}^{2} / \epsilon<b^{2}$, let $\epsilon_{d}, \epsilon \in(0,1]$, and define $\delta \mathrm{w}=\mathrm{w}-\widehat{\mathrm{w}}$ and $\delta \widetilde{\mathrm{w}}=\widetilde{\mathrm{w}}-\widetilde{\mathrm{w}}$. Forming the difference between the linearized equations, we obtain that

$$
\overline{\mathrm{A}}_{0}(\widehat{\mathrm{w}}) \partial_{t} \delta \widetilde{\mathrm{w}}+\sum_{i \in \mathcal{D}} \overline{\mathrm{A}}_{i}(\widehat{\mathrm{w}}) \partial_{i} \delta \widetilde{\mathrm{w}}-\epsilon_{d} \sum_{i, j \in \mathcal{D}} \overline{\mathrm{B}}_{i j}(\widehat{\mathrm{w}}) \partial_{i} \partial_{j} \delta \widetilde{\mathrm{w}}+\frac{1}{\epsilon} \overline{\mathrm{L}}(\widehat{\mathrm{w}}) \delta \widetilde{\mathrm{w}}=\delta \mathrm{f}+\epsilon_{d} \delta \mathrm{g} .
$$

Here

$$
\begin{gathered}
\delta \mathrm{f}=-\sum_{i \in \mathcal{D}}\left(\overline{\mathrm{A}}_{0}(\widehat{\mathrm{w}})\left(\overline{\mathrm{A}}_{0}(\mathrm{w})\right)^{-1} \overline{\mathrm{A}}_{i}(\mathrm{w})-\overline{\mathrm{A}}_{i}(\widehat{\mathrm{w}})\right) \partial_{i} \widetilde{\mathrm{w}} \\
-\frac{1}{\epsilon}\left(\overline{\mathrm{A}}_{0}(\widehat{\mathrm{w}})\left(\overline{\mathrm{A}}_{0}(\mathrm{w})\right)^{-1} \overline{\mathrm{L}}(\mathrm{w})-\overline{\mathrm{L}}(\widehat{\mathrm{w}})\right) \pi \widetilde{\mathrm{w}}, \\
\delta \mathrm{g}=\overline{\mathrm{A}}_{0}(\widehat{\mathrm{w}})\left(\overline{\mathrm{A}}_{0}(\mathrm{w})\right)^{-1} \mathrm{~g}\left(\mathrm{w}, \partial_{\times} \mathrm{w}\right)-\mathrm{g}\left(\widehat{\mathrm{w}}, \partial_{\times} \widehat{\mathrm{w}}\right) \\
+\sum_{i, j \in \mathcal{D}}\left(\overline{\mathrm{A}}_{0}(\widehat{\mathrm{w}})\left(\overline{\mathrm{A}}_{0}(\mathrm{w})\right)^{-1} \overline{\mathrm{B}}_{i j}(\mathrm{w})-\overline{\mathrm{B}}_{i j}(\widehat{\mathrm{w}})\right) \partial_{i} \partial_{j} \widetilde{\mathrm{w}},
\end{gathered}
$$

and we have in particular $\delta \mathrm{g}_{\mathrm{I}}=0$. These expressions imply that

$$
\begin{gathered}
\left|\delta \mathrm{f}_{\mathrm{I}}\right|_{l-1}^{2}+\left|\delta \mathrm{f}_{\mathrm{II}}\right|_{l-1}^{2} \leq \frac{\mathrm{c}_{2}}{\epsilon}|\delta \mathrm{w}|_{l-1}^{2}, \\
\left|\delta \mathrm{g}_{\mathrm{II}}\right|_{l-2}^{2} \leq \mathrm{c}_{2}|\delta \mathrm{w}|_{l-1}^{2},
\end{gathered}
$$

where the $1 / \epsilon$ factor arises from the nonlinear stiff sources and will make the convergence of the successive approximations more difficult to establish than for non stiff problems. We define $N_{l-1}^{2}\left(a, a^{\prime}, \delta \widetilde{\mathrm{w}}\right)$ when $\left[a, a^{\prime}\right] \subset[0, \bar{\tau}]$ by

$$
\begin{aligned}
& N_{l-1}^{2}\left(a, a^{\prime}, \delta \widetilde{\mathrm{w}}\right)=\sup _{a \leq \tau \leq a^{\prime}}\left(|\delta \widetilde{\mathrm{w}}(\tau)|_{l-1}^{2}+\frac{1}{\epsilon}|\pi \delta \widetilde{\mathrm{w}}(\tau)|_{l-2}^{2}\right)+\epsilon_{d} \int_{a}^{a^{\prime}}\left|\delta \widetilde{\mathrm{w}}_{\mathrm{II}}(\tau)\right|_{l}^{2} d \tau \\
& +\frac{1}{\epsilon} \int_{a}^{a^{\prime}}|\pi \delta \widetilde{\mathrm{w}}(\tau)|_{l-1}^{2} d \tau+\frac{1}{\epsilon^{2}} \int_{a}^{a^{\prime}}|\pi \delta \widetilde{\mathrm{w}}(\tau)|_{l-2}^{2} d \tau+\int_{a}^{a^{\prime}}\left|\partial_{t} \delta \widetilde{\mathrm{w}}(\tau)\right|_{l-2}^{2} d \tau .
\end{aligned}
$$


In order to obtain fixed points, we introduce the sequence of successive approximations $\left\{\mathrm{w}^{k}\right\}_{k>0}$ starting at $\mathrm{w}^{0}=\mathrm{w}^{\star}$ with $\mathrm{w}^{k+1}=\widetilde{\mathrm{w}}^{k}$, i.e., $\mathrm{w}^{k+1}$ is obtained as the solution $\widetilde{\mathrm{w}}=\mathrm{w}^{k+1}$ of linearized equations with $\mathrm{w}=\mathrm{w}^{k}$ and with initial condition $\mathrm{w}_{0}$. We also denote by $\delta^{k} \mathrm{w}$ the difference $\delta^{k} \mathrm{w}=$ $\mathrm{w}^{k+1}-\mathrm{w}^{k}$ for $k \geq 0$. We first establish that the sequence of successive approximations $\left\{\mathrm{w}^{k}\right\}_{k>0}$ is convergent for the norm $N_{l-1}\left(0, \tau_{\epsilon}, \bullet\right)$ and thus also for the norm of $C^{0}\left(\left[0, \tau_{\epsilon}\right], H^{l-1}\right)$ over $\left[0, \tau_{\epsilon}\right]$ for a suitable $\tau_{\epsilon}$ small enough and we then gradually extend the convergence domain over each interval $\left[j \tau_{\epsilon},(j+1) \tau_{\epsilon}\right] \subset[0, \bar{\tau}]$ by induction on $j$. We also establish uniqueness of solutions first over $\left[0, \tau_{\epsilon}\right]$ and gradually over each $\left[j \tau_{\epsilon},(j+1) \tau_{\epsilon}\right]$ included in $[0, \bar{\tau}]$.

Using the linearized estimates and the difference equation (3.24) we first obtain

$$
N_{l-1}^{2}\left(0, \tau_{\epsilon}, \delta \widetilde{\mathrm{w}}\right) \leq \mathrm{c}_{2}\left(\left|\delta \mathrm{w}_{0}\right|_{l-1}^{2}+\frac{1}{\epsilon}\left|\pi \delta \mathrm{w}_{0}\right|_{l-2}^{2}\right)+\frac{\tau_{\epsilon} \mathrm{c}_{2}^{\prime}}{\epsilon} \sup _{0 \leq \tau \leq \tau_{\epsilon}}|\delta \mathrm{w}(\tau)|_{l-1}^{2}
$$

where $c_{2}$ and $c_{2}^{\prime}$ depends on $\mathcal{O}_{1}$ and $b$ and where $\delta \mathrm{w}_{0}=\mathrm{w}_{0}-\widehat{\mathrm{w}}_{0}$. Let now $\tau_{\epsilon}$ be small enough such that

$$
\frac{\mathrm{c}_{2}^{\prime} \tau_{\epsilon}}{\epsilon}<\frac{1}{4}
$$

while $\bar{\tau} / \tau_{\epsilon}$ is an integer denoted by $N_{\epsilon}+1$. From the estimates (3.28) and since the successive approximations have the same initial condition, that is $\delta \mathrm{w}_{0}=0$, we obtain that

$$
N_{l-1}^{2}\left(0, \tau_{\epsilon}, \delta^{k+1} \mathrm{w}\right) \leq \frac{1}{4} N_{l-1}^{2}\left(0, \tau_{\epsilon}, \delta^{k} \mathrm{w}\right),
$$

so that $N_{l-1}^{2}\left(0, \tau_{\epsilon}, \delta^{k} \mathrm{w}\right) \leq N_{l-1}^{2}\left(0, \tau_{\epsilon}, \delta^{0} \mathrm{w}\right) / 4^{k}$ and defining for convenience $\Gamma^{0}=N_{l-1}^{2}\left(0, \tau_{\epsilon}, \delta^{0} \mathbf{w}\right)$, we have established that $4^{k} N_{l-1}^{2}\left(0, \tau_{\epsilon}, \delta^{k} \mathrm{~W}\right) \leq \Gamma^{0}$. Any fixed point $\overline{\mathrm{w}}$ is also unique from the difference estimates (3.28) written between two fixed points.

Letting for convenience

$$
\beta_{k}^{j}=N_{l-1}^{2}\left(j \tau_{\epsilon},(j+1) \tau_{\epsilon}, \delta^{k} \mathbf{w}\right), \quad 0 \leq k, \quad 0 \leq j \leq N_{\epsilon},
$$

we have established that $2^{k} \beta_{k}^{0} \leq \Gamma^{0}$, for $k \geq 0$, and we next consider the interval $\left[\tau_{\epsilon}, 2 \tau_{\epsilon}\right]$. From the difference equations and the linearized estimates over $\left[\tau_{\epsilon}, 2 \tau_{\epsilon}\right]$ we obtain that

$$
N_{l-1}^{2}\left(\tau_{\epsilon}, 2 \tau_{\epsilon}, \delta^{k+1} \mathbf{w}\right) \leq \mathrm{c}_{2} N_{l-1}^{2}\left(0, \tau_{\epsilon}, \delta^{k+1} \mathbf{w}\right)+\frac{\tau_{\epsilon} \mathrm{c}_{2}^{\prime}}{\epsilon} N_{l-1}^{2}\left(\tau_{\epsilon}, 2 \tau_{\epsilon}, \delta^{k} \mathbf{w}\right),
$$

where we have used that $\left|\delta \widetilde{\mathrm{w}}\left(\tau_{\epsilon}\right)\right|_{l-1}^{2}+\frac{1}{\epsilon}\left|\pi \delta \widetilde{\mathrm{w}}\left(\tau_{\epsilon}\right)\right|_{l-2}^{2} \leq N_{l-1}^{2}\left(0, \tau_{\epsilon}, \delta^{k+1} \mathrm{w}\right)$. This now implies that $\beta_{k+1}^{1} \leq \mathrm{c}_{2} \beta_{k+1}^{0}+\frac{1}{4} \beta_{k}^{1}$ for $k \geq 0$ and multiplying by $2^{k+1}$ and letting

$$
\gamma_{k}^{j}=2^{k} \beta_{k}^{j}, \quad 0 \leq k, \quad 0 \leq j \leq N_{\epsilon},
$$

we have

$$
\gamma_{k+1}^{1} \leq \mathrm{c}_{2} \gamma_{k+1}^{0}+\frac{1}{2} \gamma_{k}^{1}, \quad 0 \leq k .
$$

Since $\gamma_{k}^{0} \leq \Gamma^{0}$ it is easily deduced from (3.30) that $\gamma_{k}^{1} \leq 2 \mathrm{c}_{2} \Gamma^{0}+\gamma_{0}^{1}$ and defining $\Gamma^{1}=2 \mathrm{c}_{2} \Gamma^{0}+\gamma_{0}^{1}$ we have $\gamma_{k}^{1} \leq \Gamma^{1}$ for $k \geq 0$. We further deduce that any fixed point $\overline{\mathrm{w}}$ is unique over $\left[0,2 \tau_{\epsilon}\right]$ since it is already unique over $\left[0, \tau_{\epsilon}\right]$ and from (3.29) rewritten between two fixed points it is also unique over the interval $\left[\tau_{\epsilon}, 2 \tau_{\epsilon}\right]$.

The same type of estimates may now be established by induction on $j$ for $1 \leq j \leq N_{\epsilon}$. More specifically, assume that for $0 \leq i \leq j-1$ we have inequalities in the form $\gamma_{k}^{i} \leq \Gamma^{i}$ where the majorizing bounds $\Gamma^{i}$ are defined by $\Gamma^{0}=N_{l-1}^{2}\left(0, \tau_{\epsilon}, \delta^{0} \mathrm{w}\right)$ and $\Gamma^{i}=2 \mathrm{c}_{2} \Gamma^{i-1}+N_{l-1}^{2}\left(i \tau_{\epsilon},(i+1) \tau_{\epsilon}, \delta^{0} \mathrm{w}\right)$ for $1 \leq i \leq j-1$, and that uniqueness of fixed points holds over $\left[0, j \tau_{\epsilon}\right]$. We then consider the sequence of approximation over $\left[j \tau_{\epsilon},(j+1) \tau_{\epsilon}\right]$. Using the difference equations and the linearized estimates over $\left[j \tau_{\epsilon},(j+1) \tau_{\epsilon}\right]$, we obtain that

$$
\begin{aligned}
N_{l-1}^{2}\left(j \tau_{\epsilon},(j+1) \tau_{\epsilon}, \delta^{k+1} \mathbf{w}\right) \leq & \mathrm{c}_{2} N_{l-1}^{2}\left((j-1) \tau_{\epsilon}, j \tau_{\epsilon}, \delta^{k+1} \mathbf{w}\right) \\
& +\frac{1}{4} N_{l-1}^{2}\left(j \tau_{\epsilon},(j+1) \tau_{\epsilon}, \delta^{k} \mathbf{w}\right)
\end{aligned}
$$


where we have used

$$
\left|\delta \widetilde{\mathrm{w}}\left(j \tau_{\epsilon}\right)\right|_{l-1}^{2}+\frac{1}{\epsilon}\left|\pi \delta \widetilde{\mathrm{w}}\left(j \tau_{\epsilon}\right)\right|_{l-2}^{2} \leq N_{l-1}^{2}\left((j-1) \tau_{\epsilon}, j \tau_{\epsilon}, \delta^{k+1} \mathbf{w}\right),
$$

so that $\beta_{k+1}^{j} \leq \mathrm{c}_{2} \beta_{k+1}^{j-1}+\frac{1}{4} \beta_{k}^{j}$. Multiplying by $2^{k+1}$ we have therefore established that

$$
\gamma_{k+1}^{j} \leq \mathrm{c}_{2} \gamma_{k+1}^{j-1}+\frac{1}{2} \gamma_{k}^{j}
$$

and since $\gamma_{k}^{j-1} \leq \Gamma^{j-1}$ it is easily deduced from (3.32) that $\gamma_{k}^{j} \leq 2 \mathrm{c}_{2} \Gamma^{j-1}+\gamma_{0}^{j}$ so that defining $\Gamma^{j}=2 \mathrm{c}_{2} \Gamma^{j-1}+\gamma_{0}^{j}=2 \mathrm{c}_{2} \Gamma^{j-1}+N_{l-1}^{2}\left(j \tau_{\epsilon},(j+1) \tau_{\epsilon}, \delta^{0} \mathrm{w}\right)$ we have established that $\gamma_{k}^{j} \leq \Gamma^{j}$ for $k \geq 0$, so that

$$
N_{l-1}^{2}\left(j \tau_{\epsilon},(j+1) \tau_{\epsilon}, \delta^{k} \mathbf{w}\right) \leq \frac{\Gamma_{j}}{2^{k}}
$$

We also deduce that any fixed point $\overline{\mathrm{w}}$ is unique over $\left[j \tau_{\epsilon},(j+1) \tau_{\epsilon}\right]$ since it is already unique over $\left[0, j \tau_{\epsilon}\right]$ and from (3.31) written between two fixed points it is also unique over $\left[j \tau_{\epsilon},(j+1) \tau_{\epsilon}\right]$.

Letting for short $\mathrm{c}_{\epsilon}=\sum_{0 \leq j \leq N_{\epsilon}} \Gamma_{j}$, it is obtained from (3.33) that

$$
N_{l-1}^{2}\left(0, \bar{\tau}, \delta^{k} \mathrm{w}\right) \leq \frac{\mathrm{c}_{\epsilon}}{2^{k}}, \quad 0 \leq k,
$$

where $\mathrm{c}_{\epsilon}$ depends on $\epsilon, \mathcal{O}_{1}, b$, and the data but is independent of $k$. We thus conclude that the sequence if successive approximation $\left\{\mathrm{w}^{k}\right\}_{k \geq 0}$ is convergent over $[0, \bar{\tau}]$ towards a fixed point $\overline{\mathrm{w}}$ for the norm $N_{l-1}\left(0, \bar{\tau}, \mathrm{w}^{k}-\overline{\mathrm{w}}\right)$. Since the sequence $\left\{\mathrm{w}^{k}\right\}_{k \geq 0}$ is bounded in the space $\mathrm{X}_{\bar{\tau}}^{l}\left(\mathcal{O}_{1}, M_{b}, M_{1 b}\right)$, it follows from standard functional analysis arguments using weakly convergent subsequences that $\overline{\mathrm{w}}$ is the unique solution of the system of partial differential equations with the required regularity.

The estimates (3.18) are next established by using the fact that the solution is a fixed point $\widetilde{w}=w$. Denoting by $w$ the solution of the nonlinear system of equations, letting

$$
M_{\mathrm{w}}^{2}=\sup _{0 \leq \tau \leq \bar{\tau}}\left|\mathrm{w}(\tau)-\mathrm{w}^{\star}(\tau)\right|_{l}^{2}+\epsilon_{d} \int_{0}^{\bar{\tau}}\left|\mathrm{w}_{\mathrm{II}}(\tau)-\mathrm{w}_{\mathrm{II}}^{\star}\right|_{l+1}^{2} d \tau+\frac{1}{\epsilon} \int_{0}^{\bar{\tau}}|\pi \mathrm{w}(\tau)|_{l}^{2} d \tau,
$$

the linearized estimate (3.14) now yields that

$$
M_{\mathrm{w}}^{2} \leq \mathrm{c}_{1}^{2} \exp \left(\mathrm{c}_{2}\left(\bar{\tau}+M_{1} \sqrt{\bar{\tau}}\right)\right)\left(\left|\mathrm{w}_{0}-\mathrm{w}^{\star}\right|_{l}^{2}+\frac{1}{\epsilon}\left|\mathrm{w}_{0}-\mathrm{w}^{\star}\right|_{0}^{2}+\bar{\tau} \epsilon_{d} \mathrm{c}_{2}^{2} M_{\mathrm{w}}^{2}\right),
$$

and since $\exp \left(\mathrm{c}_{2}\left(\bar{\tau}+M_{1} \sqrt{\bar{\tau}}\right)\right) \leq 2$ and $2 \mathrm{c}_{1}^{2} \mathrm{c}_{2}^{2} \bar{\tau} \leq 1 / 2$ by definition of $\bar{\tau}$ we get that

$$
M_{\mathrm{w}}^{2} \leq C\left(\left|\mathrm{w}_{0}-\mathrm{w}^{\star}\right|_{l}^{2}+\frac{1}{\epsilon}\left|\mathrm{w}_{0}-\mathrm{w}^{\star}\right|_{0}^{2}\right)
$$

where $C$ only depends on $\mathcal{O}_{1}$ and $b$. On the other hand, from the linearized estimates (3.15) we further get that

$$
\begin{array}{r}
\frac{1}{\epsilon} \sup _{0 \leq \tau \leq \bar{\tau}}|\pi \widetilde{\mathrm{w}}(\tau)|_{l-1}^{2}+\frac{1}{\epsilon^{2}} \int_{0}^{\bar{\tau}}|\pi \widetilde{\mathrm{w}}(\tau)|_{l-1}^{2} d \tau+\int_{0}^{\bar{\tau}}\left|\partial_{t} \widetilde{\mathrm{w}}(\tau)\right|_{l-1}^{2} d \tau \\
\leq \mathrm{c}_{2} \exp \left(\mathrm{c}_{2}\left(\bar{\tau}+M_{1} \sqrt{\bar{\tau}}\right)\right)\left(\left|\mathrm{w}_{0}-\mathrm{w}^{\star}\right|_{l}^{2}+\frac{1}{\epsilon}\left|\mathrm{w}_{0}-\mathrm{w}^{\star}\right|_{l-1}^{2}+\bar{\tau} \epsilon_{d} M_{\mathrm{w}}^{2}\right) .
\end{array}
$$

Combining (3.35) and (3.36) finally yields (3.18) and the proof is complete.

\subsection{Stronger time derivative estimates}

We now strengthen the estimates for $\partial_{t} \mathrm{w}$ obtained with the local existence theorem. Such extra estimates for $\partial_{t} \mathrm{w} \in C^{0}\left([0, \bar{\tau}], H^{l-2}\right)$ and $\pi \partial_{t} \mathrm{w} / \epsilon \in L^{2}\left((0, \bar{\tau}), H^{l-3}\right)$, uniformly in $\epsilon_{d}, \epsilon \in(0,1]$ when $l \geq[d / 2]+4$ are relevant when the initial time derivative $\partial_{t} \mathrm{w}_{0}$ at $t=0$ is close to the equilibrium manifold and will be needed in the convergence analysis of the fast relaxation limit. 
Theorem 3.4. Keep the assumptions of Theorem 3.3 and further assume that $l \geq l_{0}+4$. There exists $C$ depending on $\mathcal{O}_{1}$ and $b$ such that the following estimates hold

$$
\begin{aligned}
\sup _{0 \leq \tau \leq \bar{\tau}} & \left(\left|\partial_{t} \mathrm{w}(\tau)\right|_{l-2}^{2}+\frac{1}{\epsilon}\left|\pi \partial_{t} \mathrm{w}(\tau)\right|_{l-3}^{2}\right)+\epsilon_{d} \int_{0}^{\bar{\tau}}\left|\partial_{t} \mathrm{w}_{\mathrm{II}}(\tau)\right|_{l-1}^{2} d \tau \\
& +\frac{1}{\epsilon} \int_{0}^{\bar{\tau}}\left|\pi \partial_{t} \mathrm{w}(\tau)\right|_{l-2}^{2} d \tau+\frac{1}{\epsilon^{2}} \int_{0}^{\bar{\tau}}\left|\pi \partial_{t} \mathrm{w}(\tau)\right|_{l-3}^{2} d \tau+\int_{0}^{\bar{\tau}}\left|\partial_{t}^{2} \mathrm{w}(\tau)\right|_{l-3}^{2} d \tau \\
& \leq C\left(\left|\mathrm{w}_{0}-\mathrm{w}^{\star}\right|_{l}^{2}+\frac{1}{\epsilon}\left|\pi \mathrm{w}_{0}\right|_{l-1}^{2}+\left|\partial_{t} \mathrm{w}_{0}\right|_{l-2}^{2}+\frac{1}{\epsilon}\left|\pi \partial_{t} \mathrm{w}_{0}\right|_{l-3}^{2}\right) .
\end{aligned}
$$

Proof. The proof in given in Appendix B.

Corollary 3.5. Keeping the assumptions of Theorem 3.4, there exists $C$ only depending on $\mathcal{O}_{1}$ and $b$ such that

$$
\frac{1}{\epsilon^{2}} \sup _{0 \leq \tau \leq t}|\pi \mathrm{w}(\tau)|_{l-2}^{2} \leq C\left(\left|\mathrm{w}_{0}-\mathrm{w}^{\star}\right|_{l}^{2}+\frac{1}{\epsilon}\left|\pi \mathrm{w}_{0}\right|_{l-1}^{2}+\left|\partial_{t} \mathrm{w}_{0}\right|_{l-2}^{2}+\frac{1}{\epsilon}\left|\pi \partial_{t} \mathrm{w}_{0}\right|_{l-3}^{2}\right)
$$

Moreover, if $\mathrm{w}$ and $\mathrm{w}^{\prime}$ corresponds to two initial conditions $\mathrm{w}_{0}$ and $\mathrm{w}_{0}^{\prime}$ as in Theorem 3.3 and if $\left|\mathrm{w}_{0}-\mathrm{w}^{\star}\right|_{l}^{2}+\frac{1}{\epsilon}\left|\pi \mathrm{w}_{0}\right|_{l-1}^{2}+\left|\partial_{t} \mathrm{w}_{0}\right|_{l-2}^{2}+\frac{1}{\epsilon}\left|\pi \partial_{t} \mathrm{w}_{0}\right|_{l-3}^{2}$ remains bounded by some constant only depending on $\mathcal{O}_{1}$ and $b$, letting $\delta \mathrm{w}=\mathrm{w}-\mathrm{w}^{\prime}$ and $\delta \mathrm{w}_{0}=\mathrm{w}_{0}-\mathrm{w}_{0}^{\prime}$, then there exists $C$ only depending on $\mathcal{O}_{1}$ and $b$ such that

$$
\begin{aligned}
& \sup _{0 \leq \tau \leq \bar{\tau}}\left(|\delta \mathrm{w}(\tau)|_{l-1}^{2}+\frac{1}{\epsilon}|\pi \delta \mathrm{w}(\tau)|_{l-2}^{2}\right)+\epsilon_{d} \int_{0}^{\bar{\tau}}\left|\delta \mathrm{w}_{\mathrm{II}}(\tau)\right|_{l}^{2} d \tau+\frac{1}{\epsilon} \int_{0}^{\bar{\tau}}|\pi \delta \mathrm{w}(\tau)|_{l-1}^{2} d \tau \\
& +\frac{1}{\epsilon^{2}} \int_{0}^{\bar{\tau}}|\pi \delta \mathrm{w}(\tau)|_{l-2}^{2} d \tau+\int_{0}^{\bar{\tau}}\left|\partial_{t} \delta \mathrm{w}(\tau)\right|_{l-2}^{2} d \tau \leq C\left(\left|\delta \mathrm{w}_{0}\right|_{l-1}^{2}+\frac{1}{\epsilon}\left|\pi \delta \mathrm{w}_{0}\right|_{l-2}^{2}\right)
\end{aligned}
$$

Proof. The new estimate (3.38) is first a consequence of Theorem 3.4 and of the relation (B.4).

In order to establish the differential estimate (3.39) we now combine the linearized estimates, the equation (3.24) for the difference $\delta \widetilde{\mathrm{w}}$ (where $\widetilde{\mathrm{w}}=\mathrm{w}, \widetilde{\widehat{w}}=\widehat{\mathrm{w}}$ and $\delta \widetilde{\mathrm{w}}=\delta \mathrm{w}$ ) and the new estimates (3.38) of $\sup _{0 \leq \tau \leq t}|\pi \mathrm{w}(\tau)|_{l-2} / \epsilon$. Keeping in mind the notation (3.27) for $N_{l-1}^{2}\left(0, \tau^{\prime}, \delta \mathrm{w}\right)$ we obtain from the difference equations (3.24) and the linearized estimates that

$$
\begin{aligned}
& N_{l-1}^{2}\left(0, \tau^{\prime}, \delta \mathrm{w}\right) \leq \mathrm{c}_{2}\left(\left|\delta \mathrm{w}_{0}\right|_{l-1}^{2}+\frac{1}{\epsilon}\left|\pi \delta \mathrm{w}_{0}\right|_{l-2}^{2}\right) \\
& \quad+c_{2}\left(\epsilon_{d} \int_{0}^{\tau^{\prime}}\left|\delta \mathrm{g}_{\mathrm{II}}\right|_{l-2}^{2} d \tau+\left\{\int_{0}^{\tau^{\prime}}|\delta \mathrm{f}|_{l-1} d \tau\right\}^{2}+\int_{0}^{\tau^{\prime}}|\delta \mathrm{f}|_{l-2}^{2} d \tau\right) .
\end{aligned}
$$

From the expressions (3.25) and (3.26) of $\delta \mathrm{f}$ and $\delta \mathrm{g}$, all terms in the right hand side are easily majorized thanks to the a priori estimates obtained in the local existence theorem except for the terms arising from the stiff sources

$$
\delta \mathrm{f}^{\prime}=-\frac{1}{\epsilon}\left(\overline{\mathrm{A}}_{0}(\widehat{\mathrm{w}})\left(\overline{\mathrm{A}}_{0}(\mathrm{w})\right)^{-1} \overline{\mathrm{L}}(\mathrm{w})-\overline{\mathrm{L}}(\widehat{\mathrm{w}})\right) \pi \mathrm{w} .
$$

The corresponding integrals are next majorized with

$$
\left\{\int_{0}^{\tau^{\prime}}\left|\delta f^{\prime}\right|_{l-1} d \tau\right\}^{2} \leq \tau^{\prime}\left\{\int_{0}^{\tau^{\prime}}\left|\delta f^{\prime}\right|_{l-1}^{2} d \tau\right\} \leq \tau^{\prime} c_{2} \sup _{\left[0, \tau^{\prime}\right]}|\delta \mathrm{w}|_{l-1}^{2},
$$

using the estimate for $\frac{1}{\epsilon^{2}} \int_{0}^{\bar{\tau}}|\pi \mathrm{w}(\tau)|_{l-1}^{2} d \tau$ from the local existence theorem and by

$$
\int_{0}^{\tau^{\prime}}\left|\delta f^{\prime}\right|_{l-2}^{2} d \tau \leq \tau^{\prime} \frac{\sup _{[0, \bar{\tau}]}|\pi \mathrm{w}|_{l-2}^{2}}{\epsilon^{2}} \sup _{\left[0, \tau^{\prime}\right]}|\delta \mathrm{w}|_{l-2}^{2} \leq \tau^{\prime} c_{2} \sup _{\left[0, \tau^{\prime}\right]}|\delta \mathrm{w}|_{l-1}^{2}
$$

since $\frac{1}{\epsilon^{2}} \sup _{[0, \bar{\tau}]}|\pi \mathrm{w}(\tau)|_{l-2}^{2}$ is bounded with the new estimates (3.38). Collecting from previous results, we have established that

$$
N_{l-1}^{2}\left(0, \tau^{\prime}, \delta \mathrm{w}\right) \leq \mathrm{c}_{2}\left(\left|\delta \mathrm{w}_{0}\right|_{l-1}^{2}+\frac{1}{\epsilon}\left|\pi \delta \mathrm{w}_{0}\right|_{l-2}^{2}\right)+\tau^{\prime} \mathrm{c}_{2} \sup _{\left[0, \tau^{\prime}\right]}|\delta \mathrm{w}|_{l-1}^{2}
$$


and assuming then that $\tau^{\prime}$ is such that $\tau^{\prime} c_{2}<\frac{1}{2}$, we obtain the estimates (3.39) over the interval $\left[0, \tau^{\prime}\right]$. Reitering the linearized estimates over the intervals $\left[j \tau^{\prime},(j+1) \tau^{\prime}\right] \subset[0, \bar{\tau}]$ whose number is independent of $\epsilon$ completes the proof.

Finally note that a priori estimates for $\pi \mathrm{w} / \epsilon$ generally improve as $\mathrm{w}_{0}$ and its time derivatives $\partial_{t}^{k} \mathrm{w}_{0}$, $k \geq 1$, are closer to the equilibrium manifold $\overline{\mathcal{E}}$. We may in particular use the extra estimates for the first time derivative itself and obtain the following new estimates.

Theorem 3.6. Keep the assumptions of Theorem 3.3 and assume that $l \geq l_{0}+6$. There exists $C$ depending on $\mathcal{O}_{1}$ and $b$ such that the following estimates hold

$$
\begin{gathered}
\sup _{0 \leq \tau \leq \bar{\tau}}\left(\left|\partial_{t}^{2} \mathrm{w}(\tau)\right|_{l-4}^{2}+\frac{1}{\epsilon}\left|\pi \partial_{t}^{2} \mathrm{w}(\tau)\right|_{l-5}^{2}\right)+\epsilon_{d} \int_{0}^{\bar{\tau}}\left|\partial_{t}^{2} \mathrm{w}_{\text {II }}(\tau)\right|_{l-3}^{2} d \tau \\
+\frac{1}{\epsilon} \int_{0}^{\bar{\tau}}\left|\pi \partial_{t}^{2} \mathrm{w}(\tau)\right|_{l-4}^{2} d \tau+\frac{1}{\epsilon^{2}} \int_{0}^{\bar{\tau}}\left|\pi \partial_{t}^{2} \mathrm{w}(\tau)\right|_{l-5}^{2} d \tau+\int_{0}^{\bar{\tau}}\left|\partial_{t}^{3} \mathrm{w}(\tau)\right|_{l-5}^{2} d \tau \\
\leq C\left(\left|\mathrm{w}_{0}-\mathrm{w}^{\star}\right|_{l}^{2}+\frac{1}{\epsilon}\left|\pi \mathrm{w}_{0}\right|_{l-1}^{2}+\left|\partial_{t} \mathrm{w}_{0}\right|_{l-2}^{2}+\frac{1}{\epsilon}\left|\pi \partial_{t} \mathrm{w}_{0}\right|_{l-3}^{2}\right. \\
\left.+\left|\partial_{t}^{2} \mathrm{w}_{0}\right|_{l-4}^{2}+\frac{1}{\epsilon}\left|\pi \partial_{t}^{2} \mathrm{w}_{0}\right|_{l-5}^{2}\right) .
\end{gathered}
$$

Corollary 3.7. Keeping the assumptions of Theorem 3.6, we have estimates in the form

$$
\begin{aligned}
\frac{1}{\epsilon^{2}} \sup _{0 \leq \tau \leq t}\left|\pi \partial_{t} \mathrm{w}(\tau)\right|_{l-4}^{2} \leq C\left(\left|\mathrm{w}_{0}-\mathrm{w}^{\star}\right|_{l}^{2}+\frac{1}{\epsilon}\left|\pi \mathrm{w}_{0}\right|_{l-1}^{2}+\left|\partial_{t} \mathrm{w}_{0}\right|_{l-2}^{2}\right. & \\
& \left.+\frac{1}{\epsilon}\left|\pi \partial_{t} \mathrm{w}_{0}\right|_{l-3}^{2}+\left|\partial_{t}^{2} \mathrm{w}_{0}\right|_{l-4}^{2}+\frac{1}{\epsilon}\left|\pi \partial_{t}^{2} \mathrm{w}_{0}\right|_{l-5}^{2}\right) .
\end{aligned}
$$

\subsection{Initial layers}

We discuss in this section the situation of ill prepared initial data. In order to bridge with the well prepared data case, it is then necessary to take into account initial layer correctors. Such correctors may notably be investigated by using composite expansions [43] but such a general study lay outside the range of the present work. More simply, in this paper, we are looking for a corrector in the form $\mathrm{w}^{0 l}(\tau, \mathrm{x})+\epsilon \mathrm{w}^{1 l}(\tau, \mathrm{x})$ where $\tau=t / \epsilon$. This corrector must decrease exponentially to zero as $\tau \rightarrow \infty$, and be such that the new variable

$$
\widehat{\mathrm{w}}=\mathrm{w}-\left(\mathrm{w}^{0 l}+\epsilon \mathrm{w}^{1 l}\right),
$$

corresponds to a well prepared data case. In other words, we want $\mathrm{w}^{0 l}$ and $\mathrm{w}^{1 l}$ to decrease exponentially with $\tau$ and $\widehat{w}$ to be such that $(1 / \epsilon)\left|\pi \widehat{\mathrm{w}}_{0}\right|_{l}^{2}$ and $(1 / \epsilon)\left|\pi \partial_{t} \widehat{\mathrm{w}}_{0}\right|_{l-1}^{2}$ remain bounded for $\epsilon \in(0,1]$. Note that the initial layer correctors are then only significant for small times since $\tau=t / \epsilon$.

At zeroth order, the initial layer is along the fast manifold $\mathrm{w}^{0 l}=\pi \mathrm{w}^{0 l}$ and is defined from the simplified system of ordinary differential equations

$$
\overline{\mathrm{A}}_{0}\left(\mathrm{w}_{0}\right) \partial_{\tau} \mathrm{w}^{0 l}+\overline{\mathrm{L}}\left(\mathrm{w}_{0}\right) \mathrm{w}^{0 l}=0,
$$

with initial condition $\mathrm{w}_{0}^{0 l}(\mathrm{x})=\pi \mathrm{w}(0, \mathrm{x})=\pi \mathrm{w}_{0}(\mathrm{x})$. The corresponding corrector $\mathrm{w}_{0}^{l}$ is given by

$$
\mathrm{w}^{0 l}=\exp \left(-\tau \overline{\mathrm{A}}_{0}^{-1}\left(\mathrm{w}_{0}\right) \overline{\mathrm{L}}\left(\mathrm{w}_{0}\right)\right) \pi \mathrm{w}_{0},
$$

where $\overline{\mathrm{A}}_{0}^{-1}$ and $\overline{\mathrm{L}}$ are evaluated at the inital value of the normal variable $\mathrm{w}_{0}=\mathrm{w}_{0}(\mathrm{x})$.

At first order, only the fast component is also taken into account $\mathrm{w}^{1 l}=\pi \mathrm{w}^{1 l}$, and we define $\mathrm{w}^{1 l}$ from the system of ordinary differential equations

$$
\overline{\mathrm{A}}_{0}\left(\mathrm{w}_{0}\right) \partial_{\tau} \mathrm{w}^{1 l}+\sigma \pi \Xi+\overline{\mathrm{L}}\left(\mathrm{w}_{0}\right) \mathrm{w}^{1 l}=0,
$$

with zero initial condition $\mathrm{w}^{1 l}(\mathrm{x})=0$. We have denoted here by $\Xi$ the time independant vector

$$
\Xi=\sum_{i \in \mathcal{D}} \overline{\mathrm{A}}_{i}\left(\mathrm{w}_{0}\right) \partial_{i} \mathrm{w}_{0}-\epsilon_{d} \sum_{i, j \in \mathcal{D}} \partial_{i}\left(\overline{\mathrm{B}}_{i j}\left(\mathrm{w}_{0}\right) \partial_{j} \mathrm{w}_{0}\right)-\epsilon_{d} \overline{\mathrm{b}}\left(\mathrm{w}_{0}, \partial_{\times} \mathrm{w}_{\text {II } 0}\right),
$$


and by $\sigma=\sigma(\tau)$ an exponential function in the form $\sigma(\tau)=\exp (-\alpha \tau)$ where $\alpha>0$ is a constant of the order of magnitude of the (bounded) spectrum of $\overline{\mathrm{A}}_{0}^{-1}\left(\mathrm{w}_{0}\right) \overline{\mathrm{L}}\left(\mathrm{w}_{0}\right)$. The equation (3.44) may again be integrated and yields the explicit solution

$$
\mathrm{w}^{1 l}=-\int_{0}^{\tau} \exp \left(-\left(\tau-\tau^{\prime}\right) \overline{\mathrm{A}}_{0}^{-1}\left(\mathrm{w}_{0}\right) \overline{\mathrm{L}}\left(\mathrm{w}_{0}\right)\right) \sigma\left(\tau^{\prime}\right) \mathrm{d} \tau^{\prime} \overline{\mathrm{A}}_{0}^{-1} \pi \Xi .
$$

This corrector $\mathrm{w}^{1 l}$ is also readily seen to decrease exponentially to zero as $\tau \rightarrow \infty$.

The initial value $\widehat{w}_{0}$ of the modified variable $\widehat{w}$ then has its projection of the fast manifold $\pi \widehat{w}_{0}$ such that

$$
\pi \widehat{\mathrm{w}}_{0}=\pi\left(\mathrm{w}_{0}-\left(\mathrm{w}_{0}^{0 l}+\epsilon \mathrm{w}_{0}^{1 l}\right)\right)=\pi\left(\mathrm{w}_{0}-\pi \mathrm{w}_{0}\right)=0
$$

and

$$
\begin{aligned}
\overline{\mathrm{A}}_{0}\left(\mathrm{w}_{0}\right) \partial_{t} \pi \widehat{\mathrm{w}}_{0} & =\pi \overline{\mathrm{A}}_{0}\left(\mathrm{w}_{0}\right) \partial_{t} \widehat{\mathrm{w}}_{0}=\pi \overline{\mathrm{A}}_{0}\left(\mathrm{w}_{0}\right)\left(\partial_{t} \mathrm{w}_{0}-\frac{1}{\epsilon}\left(\partial_{\tau} \mathrm{w}_{0}^{0 l}+\epsilon \partial_{\tau} \mathrm{w}_{0}^{1 l}\right)\right) \\
& =\pi\left(-\Xi-\frac{1}{\epsilon} \overline{\mathrm{L}}\left(\mathrm{w}_{0}\right) \mathrm{w}_{0}-\frac{1}{\epsilon}\left(-\overline{\mathrm{L}}\left(\mathrm{w}_{0}\right) \mathrm{w}_{0}-\epsilon \pi \Xi\right)\right)=0,
\end{aligned}
$$

so that $(1 / \epsilon)\left|\pi \widehat{\mathrm{w}}_{0}\right|_{l}^{2}$ and $(1 / \epsilon)\left|\pi \partial_{t} \widehat{\mathrm{w}}_{0}\right|_{l-1}^{2}$ are bounded. This shows that the transformed problem in terms of the modified variable $\widehat{w}$ corresponds to the situation of well prepared data.

\section{Convergence analysis and volume viscosity}

Denoting by $\varphi$ the linear operator

$$
\boldsymbol{\varphi}=\left[\begin{array}{cccc}
1 & 0_{1, d} & 0 & 0 \\
0_{d, 1} & \boldsymbol{I} & 0_{d, 1} & 0 \\
0 & 0_{1, d} & 0 & 1
\end{array}\right],
$$

the 'equilibrium components' $\varphi \mathrm{w}$ of the normal variable $\mathrm{w}$ are given by $\varphi \mathrm{w}=(\rho, \boldsymbol{v},-1 / T)^{t}$. We first estimate in this section to what extend this equilibrium projection $\varphi \mathrm{w}$ of the normal variable $w$ out of equilibrium satisfies the equations of the normal variable $w_{e}$ at thermodynamic equilibrium. We then restate a stability theorem for the equations governing fluids at thermodynamic equilibrium. By combining these results, we rigorously establish that the difference $\varphi \mathrm{w}-\mathrm{w}_{\mathrm{e}}$ is $\mathcal{O}\left(\epsilon\left(\epsilon+\epsilon_{d}\right)\right)$. This yields a convergence theorem for the fast relaxation limit as well as a rigorous justification of the volume viscosity term appearing in the equilibrium fluid model.

\subsection{Residual estimates}

The equations governing fluids at thermodynamic equilibrium have been investigated in Section 2.7 and the corresponding equations in normal form read

$$
\overline{\mathrm{A}}_{0}^{\mathrm{e}}\left(\mathrm{w}_{\mathrm{e}}\right) \partial_{t} \mathrm{w}_{\mathrm{e}}+\sum_{i \in \mathcal{D}} \overline{\mathrm{A}}_{i}^{\mathrm{e}}\left(\mathrm{w}_{\mathrm{e}}\right) \partial_{i} \mathrm{w}_{\mathrm{e}}-\epsilon_{d} \sum_{i, j \in \mathcal{D}} \partial_{i}\left(\overline{\mathrm{B}}_{i j}^{\mathrm{e}}\left(\mathrm{w}_{\mathrm{e}}\right) \partial_{j} \mathrm{w}_{\mathrm{e}}\right)-\epsilon_{d} \overline{\mathrm{b}}_{\mathrm{e}}\left(\mathrm{w}_{\mathrm{e}}, \partial_{\mathrm{x}} \mathrm{w}_{\mathrm{eII}}\right)=0
$$

where $\mathrm{w}_{\mathrm{e}}=\left(\rho_{\mathrm{e}}, \boldsymbol{v}_{\mathrm{e}},-1 / T_{\mathrm{e}}\right)^{t}$ is the normal variable at equilibrium. Moreover, using the ChapmanEnskog expansion, it has been established in our previous work [25] that the equilibrium projection $\varphi \mathrm{w}=(\rho, \boldsymbol{v},-1 / T)^{t}$ of the normal variable out of equilibrium $\mathrm{w}$ is formally a second order approximate solution of the one-temperature governing equations when $\epsilon_{d}=\epsilon$.

We estimate rigorously in this section the residual $\mathrm{h}$ defined by

$$
\begin{aligned}
\overline{\mathrm{A}}_{0}^{\mathrm{e}}(\varphi \mathrm{w}) \partial_{t}(\varphi \mathrm{w})+\sum_{i \in \mathcal{D}} \overline{\mathrm{A}}_{i}^{\mathrm{e}}(\varphi \mathrm{w}) \partial_{i}(\varphi \mathrm{w})-\epsilon_{d} & \sum_{i, j \in \mathcal{D}} \partial_{i}\left(\overline{\mathrm{B}}_{i j}^{\mathrm{e}}(\varphi \mathrm{w}) \partial_{j}(\varphi \mathrm{w})\right) \\
& -\epsilon_{d} \overline{\mathrm{b}}_{\mathrm{e}}\left(\varphi \mathrm{w}, \varphi \partial_{\times} \mathrm{w}_{\mathrm{II}}\right)=\mathrm{h},
\end{aligned}
$$


that is, we estimate the 'default to equilibrium residual' of the projection $\varphi \mathrm{w}$. Using estimates for $\mathrm{h}$ we will deduce in the next section estimates for the difference $\varphi w-w_{e}$ and establish a convergence theorem in the fast relaxation limit.

We begin by evaluating $\mathrm{h}$ in terms of fluid properties and this requires a few notation and technical lemmas. We denote by $\theta$ the reduced temperature difference

$$
\theta=\frac{T_{\mathrm{tr}}-T_{\mathrm{in}}}{\epsilon}=-T_{\mathrm{tr}} T_{\mathrm{in}} \frac{\pi \mathrm{w}}{\epsilon},
$$

which thus essentially corresponds to the fast variable $\pi \mathrm{w} / \epsilon$. The estimates for hyperbolic-parabolic systems with stiff sources obtained in Section 3 yield in particular various estimates of $\theta$ uniformly with respect to the parameters $\epsilon, \epsilon_{d} \in(0,1]$. We first investigate the difference $\phi\left(T_{\mathrm{tr}}, T_{\mathrm{in}}\right)-\phi(T, T)$ for a smooth function of the translational and internal temperatures.

Lemma 4.1. Let $\phi$ be a $C^{\varkappa}$ function $\phi\left(T_{\mathrm{tr}}, T_{\mathrm{in}}\right)$ of both temperatures $T_{\mathrm{tr}}$ and $T_{\mathrm{in}}$. Then

$$
\phi\left(T_{\mathrm{tr}}, T_{\mathrm{in}}\right)-\phi(T, T)=\epsilon \overline{\mathrm{h}}_{\phi}\left(\theta, T_{\mathrm{tr}}, T_{\mathrm{in}}\right),
$$

where $\overline{\mathrm{h}}_{\phi}$ is a $C^{\varkappa-1}$ function of $T_{\mathrm{tr}}$ and $T_{\mathrm{in}}$ and is also proportional to $\theta$.

Proof. We may first write

$$
\overline{\mathrm{h}}_{\phi}=\frac{T_{\mathrm{tr}}-T}{\epsilon} \int_{0}^{1} \partial_{T_{\mathrm{tr}}} \phi\left(T+s\left(T_{\mathrm{tr}}-T\right), T\right) \mathrm{d} s+\frac{T_{\mathrm{in}}-T}{\epsilon} \int_{0}^{1} \partial_{T_{\mathrm{in}}} \phi\left(T_{\mathrm{tr}}, T+s\left(T_{\mathrm{in}}-T\right)\right) \mathrm{d} s,
$$

and since $T_{\mathrm{tr}}-T=\left(T_{\mathrm{tr}}-T_{\mathrm{in}}\right) \widetilde{c}_{\mathrm{in}} / \widetilde{c}_{\mathrm{v}}$ and $\left(T_{\mathrm{in}}-T\right)=-\left(T_{\mathrm{tr}}-T_{\mathrm{in}}\right) c_{\mathrm{v}, \mathrm{tr}} / \widetilde{c}_{\mathrm{v}}$ we obtain that

$$
\overline{\mathrm{h}}_{\phi}=\theta\left\{\frac{\widetilde{c}_{\mathrm{in}}}{\widetilde{c}_{\mathrm{v}}} \int_{0}^{1} \partial_{T_{\mathrm{tr}}} \phi\left(T+s\left(T_{\mathrm{tr}}-T\right), T\right) \mathrm{d} s-\frac{c_{\mathrm{v}, \mathrm{tr}}}{\widetilde{c}_{\mathrm{v}}} \int_{0}^{1} \partial_{T_{\mathrm{in}}} \phi\left(T_{\mathrm{tr}}, T+s\left(T_{\mathrm{in}}-T\right)\right) \mathrm{d} s\right\} .
$$

Finally $\overline{\mathrm{h}}_{\phi}$ is $C^{\varkappa-1}$ since $\widetilde{c}_{\text {in }}$ and $\widetilde{c}_{\mathrm{v}}$ are $C^{\varkappa-1}$ functions of $\left(T_{\mathrm{tr}}, T_{\mathrm{in}}\right)$, since $T$ is a $C^{\varkappa}$ function of $\left(T_{\mathrm{tr}}, T_{\mathrm{in}}\right)$ and since $\phi$ is $C^{\varkappa}$.

We may now use this lemma in order to rewrite in a convenient form the various transport coefficients appearing in the governing equations. Keeping in mind that the viscosity $\eta$ is given by $\eta=\epsilon_{d} \bar{\eta}$ from (2.11), and using Lemma 4.1 with $\phi=\bar{\eta}$, we obtain that $\bar{\eta}\left(T_{\mathrm{tr}}, T_{\mathrm{in}}\right)-\bar{\eta}_{\mathrm{e}}(T)=\epsilon \overline{\mathrm{h}}_{\bar{\eta}}$ where $\bar{\eta}_{\mathrm{e}}(T)=\bar{\eta}(T, T)$. Further defining the total conductivity as $\lambda=\lambda_{\text {tr,tr }}+\lambda_{\text {tr,in }}+\lambda_{\text {in,tr }}+\lambda_{\text {in ,in }}$, we may write from (2.11) that $\lambda=\epsilon_{d} \bar{\lambda}$ where $\bar{\lambda}=\bar{\lambda}_{\mathrm{tr}, \mathrm{tr}}+\bar{\lambda}_{\mathrm{tr}, \mathrm{in}}+\bar{\lambda}_{\mathrm{in}, \mathrm{tr}}+\bar{\lambda}_{\mathrm{in}, \mathrm{in}}$, and using Lemma 4.1 with $\phi=\bar{\lambda}$, we then have $\bar{\lambda}\left(T_{\text {tr }}, T_{\text {in }}\right)-\bar{\lambda}_{\mathrm{e}}(T)=\epsilon \overline{\mathrm{h}}_{\bar{\lambda}}$ where $\bar{\lambda}_{\mathrm{e}}(T)=\bar{\lambda}(T, T)$. Finally, the volume viscosity may be written $\kappa=\epsilon \bar{\kappa}$ from (2.14) and using Lemma 4.1 with $\phi=\bar{\kappa}$ yields $\bar{\kappa}\left(T_{\mathrm{tr}}, T_{\mathrm{in}}\right)-\bar{\kappa}_{\mathrm{e}}(T)=\epsilon \overline{\mathrm{h}}_{\bar{\kappa}}$ where $\bar{\kappa}_{\mathrm{e}}(T)=\bar{\kappa}(T, T)$. The transport coefficients are thus in the form

$$
\begin{aligned}
& \kappa\left(T_{\mathrm{tr}}, T_{\mathrm{in}}\right)-\kappa_{\mathrm{e}}(T)=\epsilon^{2} \overline{\mathrm{h}}_{\bar{\kappa}}, \quad \eta\left(T_{\mathrm{tr}}, T_{\mathrm{in}}\right)- \eta_{\mathrm{e}}(T)=\epsilon \epsilon_{d} \overline{\mathrm{h}}_{\bar{\eta}}, \\
& \lambda\left(T_{\mathrm{tr}}, T_{\mathrm{in}}\right)-\lambda_{\mathrm{e}}(T)=\epsilon \epsilon_{d} \overline{\mathrm{h}}_{\bar{\lambda}},
\end{aligned}
$$

with $\kappa_{\mathrm{e}}(T)=\epsilon \bar{\kappa}_{\mathrm{e}}(T), \eta_{\mathrm{e}}(T)=\epsilon_{d} \bar{\eta}_{\mathrm{e}}(T)$, and $\lambda_{\mathrm{e}}(T)=\epsilon \bar{\lambda}_{\mathrm{e}}(T)$. We further define the reduced transport fluxes

$$
\begin{gathered}
\overline{\boldsymbol{Q}}_{\mathrm{tr}}=-\bar{\lambda}_{\mathrm{tr}, \mathrm{tr}} \boldsymbol{\nabla} T_{\mathrm{tr}}-\bar{\lambda}_{\mathrm{tr}, \mathrm{in}} \boldsymbol{\nabla} T_{\mathrm{in}}, \quad \overline{\boldsymbol{Q}}_{\mathrm{in}}=-\bar{\lambda}_{\mathrm{in}, \mathrm{tr}} \boldsymbol{\nabla} T_{\mathrm{tr}}-\bar{\lambda}_{\mathrm{in}, \mathrm{in}} \boldsymbol{\nabla} T_{\mathrm{in}}, \\
\overline{\boldsymbol{Q}}=\overline{\boldsymbol{Q}}_{\mathrm{tr}}+\overline{\boldsymbol{Q}}_{\mathrm{in}}, \quad \overline{\boldsymbol{\Pi}}=-\bar{\eta}\left(\boldsymbol{\nabla} \boldsymbol{v}+(\boldsymbol{\nabla} \boldsymbol{v})^{t}-\frac{2}{d^{\prime}}(\boldsymbol{\nabla} \cdot \boldsymbol{v}) \boldsymbol{I}\right),
\end{gathered}
$$

in such a way that

$$
\boldsymbol{Q}=\epsilon_{d} \overline{\boldsymbol{Q}}, \quad \boldsymbol{Q}_{\mathrm{tr}}=\epsilon_{d} \overline{\boldsymbol{Q}}_{\mathrm{tr}}, \quad \boldsymbol{Q}_{\mathrm{in}}=\epsilon_{d} \overline{\boldsymbol{Q}}_{\mathrm{in}}, \quad \boldsymbol{\Pi}=\epsilon_{d} \overline{\boldsymbol{\Pi}} .
$$

From the relation (2.15) we next obtain

$$
\begin{aligned}
\rho r\left(T_{\mathrm{tr}}-T\right)=-\kappa_{\mathrm{e}}(T) \boldsymbol{\nabla} \cdot \boldsymbol{v}-\epsilon^{2}\left(\overline{\mathrm{h}}_{\bar{\kappa}} \boldsymbol{\nabla} \cdot \boldsymbol{v}+\frac{\bar{\kappa}}{r T_{\mathrm{tr}}}\left(\partial_{t} \theta+\boldsymbol{v} \cdot \boldsymbol{\nabla} \theta\right)\right) \\
-\epsilon \epsilon_{d} \frac{\bar{\kappa}}{p}\left(\overline{\boldsymbol{\Pi}}: \boldsymbol{\nabla} \boldsymbol{v}+\boldsymbol{\nabla} \cdot \overline{\boldsymbol{Q}}_{\mathrm{tr}}-\frac{c_{\mathrm{v}, \mathrm{tr}}}{c_{\mathrm{in}}} \boldsymbol{\nabla} \cdot \overline{\boldsymbol{Q}}_{\mathrm{in}}\right),
\end{aligned}
$$

and we are ready to investigate the structure of the residual $h$. 
Proposition 4.2. The residual $\mathrm{h}$ may be written in the form

$$
\mathrm{h}=\epsilon^{2} \mathrm{~h}_{r}+\epsilon \epsilon_{d} \mathrm{~h}_{d}
$$

with

$$
\mathrm{h}_{r}=\left(\left(\partial_{\mathrm{w}_{\mathrm{e}}} \mathrm{v}_{\mathrm{e}}\right)(\varphi \mathrm{w})\right)^{t} \sum_{i \in \mathcal{D}} \partial_{i} \mathrm{~h}_{r, i}, \quad \mathrm{~h}_{d}=\left(\left(\partial_{\mathrm{w}_{\mathrm{e}}} \mathrm{v}_{\mathrm{e}}\right)(\varphi \mathrm{w})\right)^{t} \sum_{i \in \mathcal{D}} \partial_{i} \mathrm{~h}_{d, i}
$$

and denoting by $\boldsymbol{\xi}=\left(\xi_{1}, \ldots, \xi_{d}\right)^{t}$ an arbitrary vector of $\mathbb{R}^{d}$, the components $\mathrm{h}_{r, i}$ and $\mathrm{h}_{d, i}$ are given by

$$
\begin{gathered}
\sum_{i \in \mathcal{D}} \xi_{i} \mathrm{~h}_{r, i}=(0, a \boldsymbol{\xi}, a \boldsymbol{v} \cdot \boldsymbol{\xi})^{t}, \\
\sum_{i \in \mathcal{D}} \xi_{i} \mathrm{~h}_{d, i}=\left(0, \overline{\mathrm{h}}_{\bar{\eta}} \mathrm{S} \cdot \boldsymbol{\xi}+b \boldsymbol{\xi}, \overline{\mathrm{h}}_{\bar{\eta}}\langle\boldsymbol{S} \boldsymbol{v}, \boldsymbol{\xi}\rangle+b \boldsymbol{v} \cdot \boldsymbol{\xi}+\overline{\mathrm{h}}_{\bar{\lambda}} \nabla T \cdot \boldsymbol{\xi}+\boldsymbol{c}\right)^{t},
\end{gathered}
$$

where

$$
\begin{gathered}
a=\overline{\mathrm{h}}_{\bar{\kappa}} \boldsymbol{\nabla} \cdot \boldsymbol{v}+\left(\bar{\kappa} / r T_{\mathrm{tr}}\right)\left(\partial_{t} \theta+\boldsymbol{v} \cdot \boldsymbol{\nabla} \theta\right), \\
b=(\bar{\kappa} / p)\left(\overline{\boldsymbol{\Pi}}: \boldsymbol{\nabla} \boldsymbol{v}+\boldsymbol{\nabla} \cdot \overline{\boldsymbol{Q}}_{\mathrm{tr}}-\left(c_{\mathrm{v}, \mathrm{tr}} / c_{\mathrm{in}}\right) \boldsymbol{\nabla} \cdot \overline{\boldsymbol{Q}}_{\mathrm{in}}\right), \\
c_{i}=\left(\bar{\lambda}_{\mathrm{tr}, \mathrm{tr}}+\bar{\lambda}_{\mathrm{in}, \mathrm{tr}}\right) \partial_{i}\left(\theta \widetilde{c}_{\mathrm{in}} / \widetilde{c}_{\mathrm{v}}\right)-\left(\bar{\lambda}_{\mathrm{tr}, \mathrm{in}}+\bar{\lambda}_{\mathrm{in}, \mathrm{in}}\right) \partial_{i}\left(\theta c_{\mathrm{v}, \mathrm{tr}} / \widetilde{c}_{\mathrm{v}}\right),
\end{gathered}
$$

and where $\mathbf{S}=\boldsymbol{\nabla} \boldsymbol{v}+(\boldsymbol{\nabla} \boldsymbol{v})^{t}-\frac{2}{d^{\prime}}(\boldsymbol{\nabla} \cdot \boldsymbol{v}) \boldsymbol{I}$.

Proof. The residual $h$ associated with the normal form is directly related to the residual $h_{u}$ associated with the conservative variable by the relation $h=\left(\partial_{w_{e}} v_{e}(\varphi w)\right)^{t} h_{u}$. It is thus sufficient to establish that

$$
\mathrm{h}_{\mathrm{u}}=\epsilon^{2} \sum_{i \in \mathcal{D}} \partial_{i} \mathrm{~h}_{r, i}+\epsilon \epsilon_{d} \sum_{i \in \mathcal{D}} \partial_{i} \mathrm{~h}_{d, i}
$$

by using the error functions $\overline{\mathrm{h}}_{\bar{\eta}}, \overline{\mathrm{h}}_{\bar{\kappa}}, \overline{\mathrm{h}}_{\bar{\lambda}}$, the reduced transport fluxes $\overline{\boldsymbol{\Pi}}, \overline{\boldsymbol{Q}}_{\mathrm{tr}}, \overline{\boldsymbol{Q}}_{\text {in }}$, the coefficients $\bar{\kappa}, \bar{\eta}$, $\bar{\lambda}$, and the expression (4.10).

In order to evaluate the residual $h_{u}$ associated with the conservative formulation, we start from the total mass, momentum and total energy conservation equations. In the momentum and energy conservation equations, the translational temperature $T_{\mathrm{tr}}$ appearing in the state law is expressed in terms of $T$ and the volume viscosity correction by using (4.10). All transport coefficients in the momentum and energy conservation equations are also expressed using Lemma 4.1. In addition, the temperatures $T_{\mathrm{tr}}$ and $T_{\mathrm{in}}$ in the heat fluxes are expressed as $T_{\mathrm{tr}}=T+\epsilon \theta \widetilde{c}_{\mathrm{in}} / \widetilde{c}_{\mathrm{v}}$ and $T_{\mathrm{in}}=T-\epsilon \theta c_{\mathrm{v}, \mathrm{tr}} / \widetilde{\mathrm{c}}_{\mathrm{v}}$.

More specifically, from (4.10) we first obtain the expression of $\mathrm{h}_{r, i}$ as well as the contributions proportional to $(\bar{\kappa} / p)\left(\overline{\boldsymbol{\Pi}}: \boldsymbol{\nabla} \boldsymbol{v}+\boldsymbol{\nabla} \cdot \overline{\boldsymbol{Q}}_{\mathrm{tr}}-\left(c_{\mathrm{v}, \mathrm{tr}} / c_{\mathrm{in}}\right) \boldsymbol{\nabla} \cdot \overline{\boldsymbol{Q}}_{\mathrm{in}}\right)$ in $\mathrm{h}_{d, i}$. The remaining contributions in $\mathrm{h}_{d, i}$ then arise from the viscous tensor which is written

$$
\boldsymbol{\Pi}=-\eta_{\mathrm{e}}(T) \mathrm{S}-\epsilon \epsilon_{d} \overline{\mathrm{h}}_{\bar{\eta}} \mathrm{S},
$$

and from the heat flux written

$$
\begin{aligned}
\boldsymbol{Q}=- & \lambda_{\mathrm{e}}(T) \boldsymbol{\nabla} T-\epsilon \epsilon_{d} \overline{\mathrm{h}}_{\bar{\lambda}} \boldsymbol{\nabla} T \\
& -\epsilon \epsilon_{d}\left(\left(\bar{\lambda}_{\mathrm{tr}, \mathrm{tr}}+\bar{\lambda}_{\mathrm{in}, \mathrm{tr}}\right) \frac{\boldsymbol{\nabla}\left(T_{\mathrm{tr}}-T\right)}{\epsilon}+\left(\bar{\lambda}_{\mathrm{tr}, \mathrm{in}}+\bar{\lambda}_{\mathrm{in}, \mathrm{in}}\right) \frac{\boldsymbol{\nabla}\left(T_{\mathrm{in}}-T\right)}{\epsilon}\right) .
\end{aligned}
$$

Using then $T_{\mathrm{tr}}=T+\epsilon \theta \widetilde{c}_{\mathrm{in}} / \widetilde{c}_{\mathrm{v}}$ and $T_{\mathrm{in}}=T-\epsilon \theta c_{\mathrm{v}, \mathrm{tr}} / \widetilde{c}_{\mathrm{v}}$ and regrouping the various error terms completes the proof.

We now estimate the residuals $\mathrm{h}_{r}$ and $\mathrm{h}_{d}$ from (4.11)-(4.12) in the functional spaces $L^{2}\left((0, \bar{\tau}), H^{l-4}\right)$ and $L^{2}\left((0, \bar{\tau}), H^{l-3}\right)$, respectively, uniformly with respect to the parameters $\epsilon, \epsilon_{d} \in(0,1]$.

Theorem 4.3. Assume that $l \geq l_{0}+4$ and that $\mathrm{w}_{0}$ is such that

$$
\left|\mathrm{w}_{0}-\mathrm{w}^{\star}\right|_{l}^{2}+\frac{1}{\epsilon}\left|\pi \mathrm{w}_{0}\right|_{l-1}^{2}+\left|\partial_{t} \mathrm{w}_{0}\right|_{l-2}^{2}+\frac{1}{\epsilon}\left|\pi \partial_{t} \mathrm{w}_{0}\right|_{l-3}^{2}
$$

is uniformly bounded independently of $\epsilon \in(0,1]$. Then the residual $\mathrm{h}_{r}$ belongs to $C^{0}\left([0, \bar{\tau}], H^{l-3}\right)$ and is bounded in $L^{2}\left((0, \bar{\tau}), H^{l-4}\right)$ independently of $\epsilon, \epsilon_{d} \in(0,1]$, and the residual $\mathrm{h}_{d}$ belongs to $C^{0}\left([0, \bar{\tau}], H^{l-3}\right)$ and is bounded in $L^{2}\left((0, \bar{\tau}), H^{l-3}\right)$ independently of $\epsilon, \epsilon_{d} \in(0,1]$. 
Proof. It is sufficient to establish the regularity property and the uniform estimates for the residuals $\sum_{i \in \mathcal{D}} \partial_{i} \mathrm{~h}_{r, i}$ and $\sum_{i \in \mathcal{D}} \partial_{i} \mathrm{~h}_{d, i}$ associated with the conservative form $\mathrm{h}_{\mathrm{u}}$ since $\mathrm{h}=\left(\partial_{\mathrm{w}_{\mathrm{e}}} \mathrm{v}_{\mathrm{e}}\right)^{t} \mathrm{~h}_{\mathrm{u}}$. It is thus sufficient to establish that $\mathrm{h}_{r, i} \in C^{0}\left([0, \bar{\tau}], H^{l-2}\right)$ is uniformly bounded in $L^{2}\left((0, \bar{\tau}), H^{l-3}\right)$ and that $\mathrm{h}_{d, i} \in C^{0}\left([0, \bar{\tau}], H^{l-2}\right)$ is uniformly bounded in $L^{2}\left((0, \bar{\tau}), H^{l-2}\right)$ for $i \in \mathcal{D}$.

Using the relations (4.13) and (4.15) for $\mathrm{h}_{r, i}$ and the relations (4.14), (4.16) and (4.17) for $\mathrm{h}_{d, i}$, where $\theta$ has been written $\left(T_{\mathrm{tr}}-T_{\mathrm{in}}\right) / \epsilon$, a direct examination of their components shows that there are all in the space $C^{0}\left([0, \bar{\tau}], H^{l-2}\right)$ since $\mathrm{w}-\mathrm{w}^{\star} \in C^{0}\left([0, \bar{\tau}], H^{l}\right), \partial_{\mathrm{x}} \mathrm{w} \in C^{0}\left([0, \bar{\tau}], H^{l-1}\right), \partial_{t} \mathrm{w} \in C^{0}\left([0, \bar{\tau}], H^{l-2}\right)$ and $\partial_{\mathrm{x}}^{2} \mathrm{w} \in C^{0}\left([0, \bar{\tau}], H^{l-2}\right)$.

On the other hand, the uniform bound for $\mathrm{h}_{r, i}$ in $L^{2}\left((0, \bar{\tau}), H^{l-3}\right)$ is a consequence of the uniform bound for first derivatives of $w$ in $L^{2}\left((0, \bar{\tau}), H^{l-1}\right)$, of the uniform bound for $\partial_{x} \theta$ in $L^{2}\left((0, \bar{\tau}), H^{l-2}\right)$ due to (3.18), and of the uniform bounds of $\partial_{t} \theta$ in $L^{2}\left((0, \bar{\tau}), H^{l-3}\right)$ obtained with (3.37). Similarly, the uniform bounds for $\mathrm{h}_{d, i}$ in $L^{2}\left((0, \bar{\tau}), H^{l-2}\right)$ are consequences of the uniform bounds for first and second derivatives in $L^{2}\left((0, \bar{\tau}), H^{l-2}\right)$, and of the uniform bounds for $\partial_{\mathrm{x}} \theta$ in $L^{2}\left((0, \bar{\tau}), H^{l-2}\right)$ due to (3.18). It is interesting to note that the most difficult term to estimate is the time derivative of the fast variable $\partial_{t} \theta$.

\subsection{Local stability at equilibrium and convergence}

We restate a local existence theorem for an abstract perturbed hyperbolic-parabolic system of partial differential equations in normal form with small second order terms and without sources [29]. This theorem is established by Kawashima [29] without the perturbing right hand side but including such perturbed terms does not present serious difficulties. These results are then applied to the limit onetemperature fluid model presented in Section 2.7. For the sake of notational simplicity we keep the notation of our previous sections even though these results are to be applied to the normal variable $\mathrm{w}_{\mathrm{e}}$ of equilibrium fluids.

Theorem 4.4. Let $d \geq 1$ and $l \geq[d / 2]+2$ be integers and let $b>0$ be given and consider the perturbed system of equations

$$
\overline{\mathrm{A}}_{0}(\mathrm{w}) \partial_{t} \mathrm{w}+\sum_{i \in \mathcal{D}} \overline{\mathrm{A}}_{i}(\mathrm{w}) \partial_{i} \mathrm{w}-\epsilon_{d} \sum_{i, j \in \mathcal{D}} \partial_{i}\left(\overline{\mathrm{B}}_{i j}(\mathrm{w}) \partial_{j} \mathrm{w}\right)-\epsilon_{d} \overline{\mathrm{b}}\left(\mathrm{w}, \partial_{\mathrm{x}} \mathrm{w}\right)=\mathrm{f}+\epsilon_{d} \mathrm{~g}
$$

where $\overline{\mathrm{b}}=-\sum_{i, j \in \mathcal{D}} \partial_{i}\left(\partial_{\mathrm{w}} \mathrm{v}\right)^{t}\left(\partial_{\mathrm{v}} \mathrm{w}\right)^{t} \overline{\mathrm{B}}_{i j} \partial_{j} \mathrm{w}$ and where for some positive $\bar{\tau}_{\mathrm{m}}>0$

$$
\begin{gathered}
\mathrm{f} \in C^{0}\left(\left[0, \bar{\tau}_{\mathrm{m}}\right], H^{l-1}\right) \cap L^{1}\left(\left[0, \bar{\tau}_{\mathrm{m}}\right], H^{l}\right), \\
\mathrm{g} \in C^{0}\left(\left[0, \bar{\tau}_{\mathrm{m}}\right], H^{l-1}\right), \quad \mathrm{g}_{\mathrm{I}}=0 .
\end{gathered}
$$

Let $\mathcal{O}_{0}$ be given such that $\overline{\mathcal{O}}_{0} \subset \mathcal{O}_{\mathrm{w}}, d_{1}$ such that $0<d_{1}<d\left(\overline{\mathcal{O}}_{0}, \partial \mathcal{O}_{\mathrm{w}}\right)$, and define

$$
\mathcal{O}_{1}=\left\{\mathrm{w} \in \mathcal{O}_{\mathrm{w}} ; d\left(\mathrm{w}, \overline{\mathcal{O}}_{0}\right)<d_{1}\right\} .
$$

There exists $\bar{\tau}$ with $0<\bar{\tau} \leq \bar{\tau}_{\mathrm{m}}$ and $\chi>0$ depending on $\mathcal{O}_{1}$ and $b$, and independent on $\epsilon_{d} \in(0,1]$, such that for any $\mathrm{w}_{0}$ with $\mathrm{w}_{0} \in \overline{\mathcal{O}}_{0}$ and any $\mathrm{f}$ and $\mathrm{g}$ satisfying (4.20)(4.21) with

$$
\left|\mathrm{w}_{0}-\mathrm{w}^{\star}\right|_{l}^{2}<b^{2}, \quad\left\{\int_{0}^{t}|\mathrm{f}|_{l} \mathrm{~d} \tau\right\}^{2}+\int_{0}^{t}|\mathrm{f}|_{l-1}^{2} \mathrm{~d} \tau<\chi b^{2}, \quad \int_{0}^{t}|\mathrm{~g}|_{l-1}^{2} \mathrm{~d} \tau<\chi b^{2},
$$

there exists a unique local solution $\mathrm{w}$ to the perturbed system (4.19) with initial condition

$$
\mathrm{w}(0, \mathrm{x})=\mathrm{w}_{0}(\mathrm{x}), \quad \mathrm{x} \in \mathbb{R}^{d},
$$

such that

$$
\mathrm{w}(t, \mathrm{x}) \in \mathcal{O}_{1}, \quad t \in[0, \bar{\tau}], \quad \mathrm{x} \in \mathbb{R}^{d},
$$

and

$$
\begin{aligned}
& \mathrm{w}_{\mathrm{I}}-\mathrm{w}_{\mathrm{I}}^{\star} \in \mathcal{C}^{0}\left([0, \bar{\tau}], H^{l}\right) \cap \mathcal{C}^{1}\left([0, \bar{\tau}], H^{l-1}\right), \\
& \mathrm{w}_{\mathrm{II}}-\mathrm{w}_{\mathrm{II}}^{\star} \in \mathcal{C}^{0}\left([0, \bar{\tau}], H^{l}\right) \cap \mathcal{C}^{1}\left([0, \bar{\tau}], H^{l-2}\right) \cap L^{2}\left((0, \bar{\tau}), H^{l+1}\right) .
\end{aligned}
$$


In addition, there exists $C>0$ only depending on $\mathcal{O}_{1}$ and $b$, such that

$$
\begin{gathered}
\sup _{0 \leq \tau \leq \bar{\tau}}\left|\mathrm{w}(\tau)-\mathrm{w}^{\star}\right|_{l}^{2}+\epsilon_{d} \int_{0}^{\bar{\tau}}\left|\mathrm{w}_{\mathrm{II}}(\tau)-\mathrm{w}_{\mathrm{II}}^{\star}\right|_{l+1}^{2} d \tau \leq \\
C\left(\left|\mathrm{w}_{0}-\mathrm{w}^{\star}\right|_{l}^{2}+\left\{\int_{0}^{\bar{\tau}}|\mathrm{f}|_{l} \mathrm{~d} \tau\right\}^{2}+\epsilon_{d} \int_{0}^{\bar{\tau}}|\mathrm{g}|_{l-1}^{2} \mathrm{~d} \tau\right), \\
\int_{0}^{t}\left|\partial_{t} \mathrm{w}(\tau)\right|_{l-1}^{2} d \tau \leq C\left(\left|\mathrm{w}_{0}-\mathrm{w}^{\star}\right|_{l}^{2}+\left\{\int_{0}^{\bar{\tau}}|\mathrm{f}|_{l} \mathrm{~d} \tau\right\}^{2}+\int_{0}^{\bar{\tau}}|\mathrm{f}|_{l-1}^{2} \mathrm{~d} \tau+\epsilon_{d} \int_{0}^{\bar{\tau}}|\mathrm{g}|_{l-1}^{2} \mathrm{~d} \tau\right) .
\end{gathered}
$$

Moreover, if $\mathrm{w}$ and $\mathrm{w}^{\prime}$ correspond to two different inital conditions and different perturbations, letting $\delta \mathrm{w}=\mathrm{w}-\mathrm{w}^{\prime}, \delta \mathrm{f}=\mathrm{f}-\mathrm{f}^{\prime}, \delta \mathrm{g}=\mathrm{g}-\mathrm{g}^{\prime}$, then

$$
\begin{aligned}
\sup _{0 \leq \tau \leq \bar{\tau}}|\delta \mathrm{w}(\tau)|_{l-1}^{2} & +\epsilon_{d} \int_{0}^{\bar{\tau}}\left|\delta \mathrm{w}_{\mathrm{II}}(\tau)\right|_{l}^{2} d \tau \\
& \leq C\left(\left|\delta \mathrm{w}_{0}\right|_{l-1}^{2}+\left\{\int_{0}^{\bar{\tau}}|\delta \mathrm{f}|_{l-1} \mathrm{~d} \tau\right\}^{2}+\epsilon_{d} \int_{0}^{\bar{\tau}}|\delta \mathrm{g}|_{l-2}^{2} \mathrm{~d} \tau\right) .
\end{aligned}
$$

Remark 4.5. We have not included nonstiff source terms in the stability theorem since they are not required for our application to fluids out of thermodynamical equilibrium. However, it is straightforward to add such nonstiff extra sources in the stability analysis.

Remark 4.6. The decomposition of the right hand side in the form $\mathbf{f}+\epsilon_{d} \mathrm{~g}$ with (4.20)(4.21) is of course not unique. A right hand side in the form $\epsilon_{d} \mathrm{~g}^{\prime}$ may be decomposed for instance into $\mathrm{f}=\epsilon_{d} \mathrm{~g}^{\prime}$ and $\mathrm{g}=0$ as well as $\mathrm{f}=0$ and $\mathrm{g}=\mathrm{g}^{\prime}$. The interest of the $\mathrm{g}$ term is that only the lower regularity $\mathrm{g}^{\prime} \in C^{0}\left(\left[0, \bar{\tau}_{\mathrm{m}}\right], H^{l-1}\right)$ is needed, but the price to pay is a factor $\sqrt{\epsilon_{d}}$ since the dissipative terms are $\mathcal{O}\left(\epsilon_{d}\right)$ and there are correspondingly $\epsilon_{d}$ factors in the estimates of higher derivatives. In particular, in the estimates (4.25), even though $\epsilon_{d} \mathrm{~g}^{\prime}$ is $\mathcal{O}\left(\epsilon_{d}\right)$, the perturbation $\delta \mathrm{w}$ is only shown to be $\mathcal{O}\left(\sqrt{\epsilon_{d}}\right)$. On the contrary, with the $\mathrm{f}$ factor, any scaling of $\mathrm{f}$ is fully tranmitted to $\delta \mathrm{w}$, but we then need the stronger relularity $\mathrm{f} \in C^{0}\left(\left[0, \bar{\tau}_{\mathrm{m}}\right], H^{l}\right)$.

We now combine the estimate of the residual $h$ to the stability theorem at equilibrium in order to obtain a convergence theorem.

Theorem 4.7. Let $d \geq 1, l \geq l_{0}+4, l_{0}=[d / 2]+1$, be integers and let $b>0$ be given. Let $\mathcal{O}_{0}$ be given such that $\overline{\mathcal{O}}_{0} \subset \mathcal{O}_{\mathrm{w}}, d_{1}$ such that $0<d_{1}<d\left(\overline{\mathcal{O}}_{0}, \partial \mathcal{O}_{\mathrm{w}}\right)$, and define $\mathcal{O}_{1}=\left\{\mathrm{w} \in \mathcal{O}_{\mathrm{w}} ; d\left(\mathrm{w}, \overline{\mathcal{O}}_{0}\right)<d_{1}\right\}$. There exists $\bar{\tau}>0$ depending on $\mathcal{O}_{1}$ and $b$, and independent on $\epsilon_{d} \in(0,1]$ and $\epsilon \in(0,1]$, such that for any $\mathrm{w}_{0} \in \overline{\mathcal{O}}_{0}$ with

$$
\left|\mathrm{w}_{0}-\mathrm{w}^{\star}\right|_{l}^{2}+\frac{1}{\epsilon}\left|\pi \mathrm{w}_{0}\right|_{l-1}^{2}<b^{2}
$$

and such that

$$
\left|\mathrm{w}_{0}-\mathrm{w}^{\star}\right|_{l}^{2}+\frac{1}{\epsilon}\left|\pi \mathrm{w}_{0}\right|_{l-1}^{2}+\left|\partial_{t} \mathrm{w}_{0}\right|_{l-2}^{2}+\frac{1}{\epsilon}\left|\pi \partial_{t} \mathrm{w}_{0}\right|_{l-3}^{2},
$$

is also bounded independently of $\epsilon$, there exists a unique solution of the out of equilibrium system such that the estimates (3.18) and (3.39) holds, as well as the estimates (3.37), and furthermore, there exists a unique solution of the equilibrium system starting from $\varphi \mathrm{w}_{0}$. Then there exists a constant $C$ depending on $\mathcal{O}_{1}$ and $b$ and independent of $\epsilon, \epsilon_{d} \in[0,1)$ such that

$$
\sup _{\tau \in[0, \bar{\tau}]}\left|\varphi \mathrm{w}-\mathrm{w}_{\mathrm{e}}\right|_{l-4} \leq C \epsilon\left(\epsilon+\epsilon_{d}\right) .
$$

Proof. We may use the estimates of the residual term $\mathrm{h}$ in Theorem 4.3 and apply Theorem 4.4 with $\mathrm{f}=\mathrm{h}, \mathrm{g}=0$, and use the differential estimates (4.25).

In particular, in the special case $\epsilon_{d}=\epsilon$, we have established that the two term Chapmen-Enskog expansion derived in the companion paper [25], which includes the $\mathcal{O}(\epsilon)$ volume viscosity terms, is effectively of second order accuracy. 


\section{Conclusion}

We have proved rigorously for the first time that the solution of the out of equilibrium gas model converges towards the solution of the one temperature model. We have further established that the distance between these two solutions is of the order of Burnett type residuals. This is in full agreement with our previous work where it has been established that the volume viscosity coefficient is obtained with a two term Chapman-Enskog expansion [25].

\section{A Linearized equations estimates}

We establish in Appendix A the proof of the linearized estimates (3.14)(3.15). It is sufficient to establish these estimates for smooth solutions since we may use mollifiers and convolution operators [29]. We follow the elegant method of proof of Kawashima [29] and mainly indicate the differences due to the stiff sources.

Step 0. Preliminaries. In the following $\delta_{1}=\delta\left(\mathcal{O}_{1}\right) \leq 1$ denotes a generic small constant only depending on $\mathcal{O}_{1}, \mathrm{c}_{1}=\mathrm{c}_{1}\left(\mathcal{O}_{1}\right) \geq 1$ a generic large constant only depending on $\mathcal{O}_{1}$, and $\mathrm{c}_{2}=\mathrm{c}_{2}\left(\mathcal{O}_{1}, M\right) \geq 1$ a generic large constant depending on $\mathcal{O}_{1}$ and $M$. The various occurrences of these constants may be distinguished and the minimum of all $\delta_{1}$ and the maxima of all $c_{1}$ and $c_{2}$ may be taken at the end of the proof so that only single constants ultimately remain. The dependence on $d, l, n$ of these estimating constants, on the other hand, is left implicit. For $k \geq 0$ and $\phi \in H^{k}$ we also define

$$
\begin{aligned}
& E_{k}^{2}(\phi)=\sum_{0 \leq|\alpha| \leq k} \frac{|\alpha| !}{\alpha !} \int_{\mathbb{R}^{d}}\left\langle\overline{\mathrm{A}}_{0}(\mathrm{w}) \partial^{\alpha} \phi, \partial^{\alpha} \phi\right\rangle \mathrm{dx}, \\
& \widehat{E}_{k}^{2}(\phi)=\sum_{0 \leq|\alpha| \leq k} \frac{|\alpha| !}{\alpha !} \int_{\mathbb{R}^{d}}\left\langle\overline{\mathrm{L}}(\mathrm{w}) \partial^{\alpha} \phi, \partial^{\alpha} \phi\right\rangle \mathrm{d} \mathrm{x} .
\end{aligned}
$$

In order to alleviate notation in the proof we denote for short $\delta \widetilde{w}=\widetilde{w}-\widetilde{w}^{\star}$. We will use the classical estimates

$$
|f(\phi)-f(0)|_{k} \leq \mathrm{c}_{0}\|f\|_{\mathcal{C}^{k}\left(\|\cdot\| \leq\|\phi\|_{\left.L^{\infty}\right)}\right.}\left(1+\|\phi\|_{L^{\infty}}\right)^{k-1}|\phi|_{k}
$$

where $k \geq 1$ and $\mathrm{c}_{0}$ denotes a generic constant independent of $\mathcal{O}_{1}$ and $M$, as well as the estimates $|u v|_{k}^{2} \leq c_{0}|u|_{l}^{2}|v|_{k}^{2}$, for $0 \leq k \leq \bar{l}$, and $\|\phi\|_{L^{\infty}} \leq \mathrm{c}_{0}|\phi|_{\bar{l}}$ valid for any $\bar{l} \geq l_{0}=[d / 2]+1$. We also have the commutator estimate $\sum_{0 \leq|\alpha| \leq l}\left|\left[\partial^{\alpha}, u\right] v\right|_{0} \leq c_{0}\left|\partial_{x} u\right|_{l-1}|v|_{l-1}$ valid for any $l \geq l_{0}+1$ where $\left[\partial^{\alpha}, u\right] v=\partial^{\alpha}(u v)-u \partial^{\alpha} v$ denotes the commutator between $\partial^{\alpha}$ and $u$. Finally, we have the Garding inequality

$$
\delta_{1}\left|\phi_{\mathrm{II}}\right|_{1}^{2} \leq \sum_{i, j \in \mathcal{D}} \int_{\mathbb{R}^{d}}\left\langle\overline{\mathrm{B}}_{i j}^{\mathrm{II}, \mathrm{II}}(\mathrm{w}) \partial_{i} \phi_{\mathrm{II}}, \partial_{j} \phi_{\mathrm{II}}\right\rangle \mathrm{dx}+\mathrm{c}_{2}\left|\phi_{\mathrm{II}}\right|_{0}^{2}
$$

for any vector valued function $\phi_{\mathrm{II}}: \mathbb{R}^{d} \rightarrow \mathbb{R}^{n_{\mathrm{II}}}$ in the space $H^{1}$.

Step 1. The zeroth order estimates. Multiplying (3.5) by $\delta \widetilde{w}=\widetilde{w}-\widetilde{w}^{\star}$ integrating over $\mathbb{R}^{d}$, using the symmetry of $\overline{\mathrm{A}}_{0}$ and $\overline{\mathrm{A}}_{i}$, using Garding inequality, and noting that $\left|\partial_{x} \mathrm{w}\right|_{l-1} \leq M$, while $c_{2}$ may depend on $M$, we obtain after some algebra that

$$
\partial_{t} E_{0}^{2}(\delta \widetilde{\mathrm{w}})+\epsilon_{d} \delta_{1}\left|\delta \widetilde{\mathrm{w}}_{\mathrm{II}}\right|_{1}^{2}+\frac{\delta_{1}}{\epsilon}|\pi \widetilde{\mathrm{w}}|_{0}^{2} \leq \mathrm{c}_{1}|\mathrm{f}|_{0}|\delta \widetilde{\mathrm{w}}|_{0}+\epsilon_{d} \mathrm{c}_{1}\left|\mathrm{~g}_{\mathrm{II}}\right|_{0}^{2}+\mathrm{c}_{2}\left(1+\left|\partial_{t} \mathrm{w}\right|_{l-1}\right) E_{0}^{2}(\delta \widetilde{\mathrm{w}}) .
$$

Letting $\gamma^{2}(t)=\sup _{0 \leq \tau \leq t} E_{0}^{2}(\delta \widetilde{\mathrm{w}}(\tau))+\delta_{1} \epsilon_{d} \int_{0}^{t}\left|\delta \widetilde{\mathrm{W}}_{\mathrm{II}}\right|_{1}^{2} d \tau+\frac{\delta_{1}}{\epsilon} \int_{0}^{t}|\pi \widetilde{\mathrm{W}}|_{0}^{2} d \tau$, noting that $\gamma$ is nondecreasing so that $\int_{0}^{t}|\mathrm{f}|_{0} \gamma(\tau) \mathrm{d} \tau \leq \gamma(t) \int_{0}^{t}|\mathrm{f}|_{0} \mathrm{~d} \tau$, and noting that $\int_{0}^{t}\left|\partial_{t} \mathrm{w}\right|_{l-1} \mathrm{~d} \tau \leq M_{1} \sqrt{t}$ we obtain from Gronwall inequality with $\gamma^{2}$ that

$$
\sup _{0 \leq \tau \leq t}|\delta \widetilde{\mathrm{w}}(\tau)|_{0}^{2}+\epsilon_{d} \int_{0}^{t}\left|\delta \widetilde{\mathrm{w}}_{\mathrm{II}}(\tau)\right|_{1}^{2} d \tau+\frac{1}{\epsilon} \int_{0}^{t}|\pi \widetilde{\mathrm{w}}|_{0}^{2} d \tau
$$




$$
\leq \mathrm{c}_{1}^{2} \exp \left(\mathrm{c}_{2}\left(t+M_{1} \sqrt{t}\right)\right)\left(\left|\delta \widetilde{\mathrm{w}}_{0}\right|_{0}^{2}+\epsilon_{d} \mathrm{c}_{1} \int_{0}^{t}\left|\mathrm{~g}_{\mathrm{II}}\right|_{0}^{2} d \tau+\mathrm{c}_{1}\left\{\int_{0}^{t}|\mathrm{f}|_{0} d \tau\right\}^{2}\right) .
$$

Similarly, multiplying the governing equation (3.5) by $(1 / \epsilon) \pi \widetilde{\mathrm{w}}$ and proceeding as above we get

$$
\frac{1}{\epsilon} \partial_{t} E_{0}^{2}(\pi \widetilde{\mathrm{w}})+\frac{\delta_{1}}{\epsilon^{2}}|\pi \widetilde{\mathrm{w}}|_{0}^{2} \leq \mathrm{c}_{1}|\pi \mathrm{f}|_{0}^{2}+\epsilon_{d}^{2} \mathrm{c}_{1}\left|\mathrm{~g}_{\mathrm{II}}\right|_{0}^{2}+\frac{\mathrm{c}_{1}}{\epsilon}\left|\partial_{t} \mathrm{w}\right|_{l-1} E_{0}^{2}(\pi \widetilde{\mathrm{w}})+\mathrm{c}_{1}|\delta \widetilde{\mathrm{w}}|_{1}^{2}+\epsilon_{d}^{2} \mathrm{c}_{1}\left|\delta \widetilde{\mathrm{w}}_{\mathrm{II}}\right|_{2}^{2} .
$$

Moreover, multiplying the governing equation by $\partial_{t} \widetilde{\mathrm{w}}$, integrating over $\mathbb{R}^{d}$, using the symmetry of $\overline{\mathrm{L}}$, and proceeding similarly we obtain that

$$
\delta_{1}\left|\partial_{t} \widetilde{\mathrm{w}}\right|_{0}^{2}+\frac{1}{\epsilon} \partial_{t} \widehat{E}_{0}^{2}(\pi \widetilde{\mathrm{w}}) \leq \mathrm{c}_{1}|\mathrm{f}|_{0}^{2}+\mathrm{c}_{1} \epsilon_{d}^{2}\left|\mathrm{~g}_{\mathrm{II}}\right|_{0}^{2}+\frac{\mathrm{c}_{1}}{\epsilon}\left|\partial_{t} \mathrm{w}\right|_{l-1} \widehat{E}_{0}^{2}(\pi \widetilde{\mathrm{w}})+\mathrm{c}_{1}|\delta \widetilde{\mathrm{w}}|_{1}^{2}+\epsilon_{d}^{2} \mathrm{c}_{1}\left|\delta \widetilde{\mathrm{w}}_{\mathrm{II}}\right|_{2}^{2}
$$

Step 2. The $l$ th order estimate. We first differentiate the hyperbolic-parabolic system (3.5) with respect to the space variable. Denoting by $\partial^{\alpha}$ the $\alpha$ th derivative spatial operator, we obtain that

$$
\overline{\mathrm{A}}_{0}(\mathrm{w}) \partial_{t} \partial^{\alpha} \widetilde{\mathrm{w}}+\sum_{i \in \mathcal{D}} \overline{\mathrm{A}}_{i}(\mathrm{w}) \partial_{i} \partial^{\alpha} \widetilde{\mathrm{w}}-\epsilon_{d} \sum_{i, j \in \mathcal{D}} \overline{\mathrm{B}}_{i j}(\mathrm{w}) \partial_{i} \partial_{j} \partial^{\alpha} \widetilde{\mathrm{w}}+\frac{1}{\epsilon} \overline{\mathrm{L}}(\mathrm{w}) \partial^{\alpha} \widetilde{\mathrm{w}}=\mathrm{f}^{\alpha}+\epsilon_{d} \mathrm{~g}^{\alpha},
$$

with

$$
\begin{gathered}
\mathrm{f}^{\alpha}=\overline{\mathrm{A}}_{0} \partial^{\alpha}\left(\overline{\mathrm{A}}_{0}^{-1} \mathrm{f}\right)-\sum_{i \in \mathcal{D}} \overline{\mathrm{A}}_{0}\left[\partial^{\alpha}, \overline{\mathrm{A}}_{0}^{-1} \overline{\mathrm{A}}_{i}\right] \partial_{i} \widetilde{\mathrm{w}}-\frac{1}{\epsilon} \overline{\mathrm{A}}_{0}\left[\partial^{\alpha}, \overline{\mathrm{A}}_{0}^{-1} \overline{\mathrm{L}}\right] \pi \widetilde{\mathrm{w}}, \\
\mathrm{g}^{\alpha}=\overline{\mathrm{A}}_{0} \partial^{\alpha}\left(\overline{\mathrm{A}}_{0}^{-1} \mathrm{~g}\right)+\sum_{i, j \in \mathcal{D}} \overline{\mathrm{A}}_{0}\left[\partial^{\alpha}, \overline{\mathrm{A}}_{0}^{-1} \overline{\mathrm{B}}_{i j}\right] \partial_{i} \partial_{j} \widetilde{\mathrm{w}} .
\end{gathered}
$$

Multiplying equation (A.6) by $\partial^{\alpha} \delta \widetilde{\mathrm{w}}$ and $|\alpha| ! / \alpha$ !, integrating over $\mathbb{R}^{d}$, summing over $0 \leq|\alpha| \leq l$, and proceeding as for the zeroth order estimate, we obtain that

$$
\begin{aligned}
\partial_{t} E_{l}^{2}(\delta \widetilde{\mathrm{w}}) & +\epsilon_{d} \delta_{1}\left|\delta \widetilde{\mathrm{w}}_{\mathrm{II}}\right|_{l+1}^{2}+\frac{\delta_{1}}{\epsilon}|\pi \widetilde{\mathrm{w}}|_{l}^{2} \leq \mathrm{c}_{2}\left(1+\left|\partial_{t} \mathrm{w}\right|_{l-1}\right) E_{l}^{2}(\delta \widetilde{\mathrm{w}}) \\
& +\sum_{0 \leq|\alpha| \leq l} \frac{|\alpha| !}{\alpha !} \int_{\mathbb{R}^{d}}\left\langle\mathrm{f}^{\alpha}, \partial^{\alpha} \delta \widetilde{\mathrm{w}}\right\rangle \mathrm{dx}+\epsilon_{d} \sum_{0 \leq|\alpha| \leq l} \frac{|\alpha| !}{\alpha !} \int_{\mathbb{R}^{d}}\left\langle\mathrm{~g}^{\alpha}, \partial^{\alpha} \delta \widetilde{\mathrm{w}}\right\rangle \mathrm{dx} .
\end{aligned}
$$

We next have to investigate the residuals associated with $\mathrm{f}^{\alpha}$ and $\mathrm{g}^{\alpha}$. Keeping in mind that the zeroth order terms with $|\alpha|=0$ have already been examined in Step1, we only have to analyze the terms such that $1 \leq|\alpha| \leq l$.

The nonstiff terms are estimated in the classical way [29] using commutator estimates (and integration by parts for the terms $\overline{\mathrm{A}}_{0} \partial^{\alpha}\left(\overline{\mathrm{A}}_{0}^{-1} \mathrm{~g}\right)$ when $\left.|\alpha|=l\right)$ and we obtain that

$$
\begin{gathered}
\left|\int_{\mathbb{R}^{d}}\left\langle\overline{\mathrm{A}}_{0} \partial^{\alpha}\left(\overline{\mathrm{A}}_{0}^{-1} \mathrm{f}\right), \partial^{\alpha} \delta \widetilde{\mathrm{w}}\right\rangle \mathrm{dx}\right| \leq\left|\overline{\mathrm{A}}_{0}\right|_{\infty}\left|\overline{\mathrm{A}}_{0}^{-1} \mathrm{f}\right|_{l}|\delta \widetilde{\mathrm{w}}|_{l} \leq \mathrm{c}_{2}|\mathrm{f}|_{l}|\delta \widetilde{\mathrm{w}}|_{l}, \\
\left|\int_{\mathbb{R}^{d}}\left\langle\overline{\mathrm{A}}_{0}\left[\partial^{\alpha}, \overline{\mathrm{A}}_{0}^{-1} \overline{\mathrm{A}}_{i}\right] \partial_{i} \widetilde{\mathrm{w}}, \partial^{\alpha} \delta \widetilde{\mathrm{w}}\right\rangle \mathrm{dx}\right| \leq\left|\overline{\mathrm{A}}_{0}\right|_{\infty}\left|\left[\partial^{\alpha}, \overline{\mathrm{A}}_{0}^{-1} \overline{\mathrm{A}}_{i}\right] \partial_{i} \widetilde{\mathrm{w}}\right|_{0}|\delta \widetilde{\mathrm{w}}|_{|\alpha|}, \\
\left|\int_{\mathbb{R}^{d}}\left\langle\overline{\mathrm{A}}_{0}\left[\partial^{\alpha}, \overline{\mathrm{A}}_{0}^{-1} \overline{\mathrm{A}}_{i}\right] \partial_{i} \widetilde{\mathrm{w}}, \partial^{\alpha} \delta \widetilde{\mathrm{w}}\right\rangle \mathrm{dx}\right| \leq \\
\mathrm{c}_{0}\left|\overline{\mathrm{A}}_{0}\right|_{\infty}\left|\partial_{\mathrm{x}}\left(\overline{\mathrm{A}}_{0}^{-1} \overline{\mathrm{A}}_{i}\right)\right|_{l-1}\left|\partial_{i} \widetilde{\mathrm{w}}\right|_{l-1}|\delta \widetilde{\mathrm{w}}|_{l} \leq \mathrm{c}_{2}|\delta \widetilde{\mathrm{w}}|_{l}^{2}, \\
\left|\int_{\mathbb{R}^{d}}\left\langle\overline{\mathrm{A}}_{0} \partial^{\alpha}\left(\overline{\mathrm{A}}_{0}^{-1} \mathrm{~g}\right), \partial^{\alpha} \delta \widetilde{\mathrm{w}}\right\rangle \mathrm{dx}\right| \leq \mathrm{c}_{2}\left|\mathrm{~g}_{\mathrm{II}}\right|_{l-1}\left|\delta \widetilde{\mathrm{w}}_{\mathrm{II}}\right|_{l+1}, \\
\left|\int_{\mathbb{R}^{d}}\left\langle\overline{\mathrm{A}}_{0}\left[\partial^{\alpha}, \overline{\mathrm{A}}_{0}^{-1} \overline{\mathrm{B}}_{i j}\right] \partial_{i} \partial_{j} \widetilde{\mathrm{w}}, \partial^{\alpha} \delta \widetilde{\mathrm{w}}\right\rangle \mathrm{dx}\right| \leq \mathrm{c}_{2}\left|\delta \widetilde{\mathrm{w}}_{\mathrm{II}}\right|_{l+1}\left|\delta \widetilde{\mathrm{w}}_{\mathrm{II}}\right|_{l} .
\end{gathered}
$$


On the other hand, for the stiff terms $\frac{1}{\epsilon} \overline{\mathrm{A}}_{0}\left[\partial^{\alpha}, \overline{\mathrm{A}}_{0}^{-1} \overline{\mathrm{L}}\right] \pi \widetilde{\mathrm{W}}$ it is obtained that

$$
\begin{aligned}
& \frac{1}{\epsilon}\left|\int_{\mathbb{R}^{d}}\left\langle\overline{\mathrm{A}}_{0}\left[\partial^{\alpha}, \overline{\mathrm{A}}_{0}^{-1} \overline{\mathrm{L}}\right] \pi \widetilde{\mathrm{w}}, \partial^{\alpha} \widetilde{\mathrm{w}}\right\rangle \mathrm{dx}\right| \leq \\
& \quad \frac{\mathrm{C}_{0}}{\epsilon}\left|\overline{\mathrm{A}}_{0}\right|_{\infty}\left|\partial_{\times}\left(\overline{\mathrm{A}}_{0}^{-1} \overline{\mathrm{L}}\right)\right|_{l-1}|\pi \widetilde{\mathrm{w}}|_{l-1}|\pi \widetilde{\mathrm{w}}|_{l} \leq \frac{\mathrm{C}_{2}}{\epsilon}|\pi \widetilde{\mathrm{W}}|_{l-1}|\pi \widetilde{\mathrm{W}}|_{l} .
\end{aligned}
$$

Collecting all contributions we have established that

$$
\begin{aligned}
\partial_{t} E_{l}^{2}(\delta \widetilde{\mathrm{w}})+\epsilon_{d} \delta_{1}\left|\delta \widetilde{\mathrm{w}}_{\mathrm{II}}\right|_{l+1}^{2}+ & \frac{\delta_{1}}{\epsilon}|\pi \widetilde{\mathrm{w}}|_{l}^{2} \leq \mathrm{c}_{2}\left(1+\left|\partial_{t} \mathrm{w}\right|_{l-1}\right) E_{l}^{2}(\delta \widetilde{\mathrm{w}}) \\
& +\mathrm{c}_{2}|\mathrm{f}|_{l} E_{l}(\delta \widetilde{\mathrm{w}})+\frac{\mathrm{c}_{2}}{\epsilon}|\pi \widetilde{\mathrm{w}}|_{l-1}^{2}+\epsilon_{d} \mathrm{c}_{2}\left|\mathrm{~g}_{\mathrm{II}}\right|_{l-1}^{2}
\end{aligned}
$$

In order to handle the term $\left(c_{2} / \epsilon\right)|\pi \widetilde{w}|_{l-1}^{2}$ in the right hand side we use the following classical consequence of interpolation inequalities $|\phi|_{l-1}^{2} \leq \beta|\phi|_{l}^{2}+C(\beta)|\phi|_{0}^{2}$ valid for any $\phi \in H^{l}$, any $\beta>0$ and where $C(\beta)$ depends on $\beta$, and we may also add the projected zeroth order inequality (A.4). Keeping in mind that $l \geq l_{0}+1 \geq 2$ we obtain that

$$
\begin{aligned}
& \partial_{t}\left(E_{l}^{2}(\delta \widetilde{\mathrm{w}})+\frac{1}{\epsilon} E_{0}^{2}(\pi \widetilde{\mathrm{w}})\right)+\epsilon_{d} \delta_{1}\left|\delta \widetilde{\mathrm{w}}_{\mathrm{II}}\right|_{l+1}^{2}+\frac{\delta_{1}}{\epsilon}|\pi \widetilde{\mathrm{w}}|_{l}^{2}+\frac{\delta_{1}}{\epsilon^{2}}|\pi \widetilde{\mathrm{w}}|_{0}^{2} \leq \\
& \mathrm{c}_{2}\left(1+\left|\partial_{t} \mathrm{w}\right|_{l-1}\right)\left(E_{l}^{2}(\delta \widetilde{\mathrm{w}})+\frac{1}{\epsilon} E_{0}^{2}(\pi \widetilde{\mathrm{w}})\right)+\mathrm{c}_{2}|\mathrm{f}|_{l} E_{l}(\delta \widetilde{\mathrm{w}})+\epsilon_{d} \mathrm{c}_{2}\left|\mathrm{~g}_{\mathrm{II}}\right|_{l-1}^{2}+\mathrm{c}_{1}|\pi \mathrm{f}|_{0}^{2}
\end{aligned}
$$

From Gronwall inequality we obtain after some algebra the first estimate (3.14). It is interesting to note that in the absence of stiff source terms we may let $\pi=0$ and we recover the usual estimates [29].

Step 3. The $l$ th order derived estimate. Multiplying the equation (A.6) by $\partial_{t} \partial^{\alpha} \widetilde{\mathbf{w}}$ and $|\alpha| ! / \alpha$ !, integrating over $\mathbb{R}^{d}$, summing over $0 \leq|\alpha| \leq l-1$, and proceeding as for the zeroth order derived estimate, we obtain that

$$
\begin{aligned}
\delta_{1}\left|\partial_{t} \widetilde{\mathrm{w}}\right|_{l-1}^{2}+\frac{1}{\epsilon} \partial_{t} \widehat{E}_{l-1}^{2}(\pi \widetilde{\mathrm{w}}) \leq \mathrm{c}_{1}|\delta \widetilde{\mathrm{w}}|_{l}^{2}+\mathrm{c}_{1} \epsilon_{d}^{2}\left|\delta \widetilde{\mathrm{w}}_{\mathrm{II}}\right|_{l+1}^{2} & +\frac{\mathrm{c}_{1}}{\epsilon}\left|\partial_{t} \mathrm{w}\right|_{l-1} \widehat{E}_{l-1}^{2}(\pi \widetilde{\mathrm{w}}) \\
& +\sum_{0 \leq|\alpha| \leq l-1} \mathrm{c}_{1} \frac{|\alpha| !}{\alpha !}\left(\left|\mathrm{f}^{\alpha}\right|_{0}^{2}+\epsilon_{d}^{2}\left|\mathrm{~g}^{\alpha}\right|_{0}^{2}\right) .
\end{aligned}
$$

and we have to investigate the residuals associated with $\mathrm{f}^{\alpha}$ and $\mathrm{g}^{\alpha}$. Keeping in mind that the zeroth order terms with $|\alpha|=0$ have already been examined in Step 3 we only have to analyze the terms such that $1 \leq|\alpha| \leq l-1$.

The nonstiff terms in $\mathrm{f}^{\alpha}$ and $\mathrm{g}^{\alpha}$ are estimated as usual whereas the stiff terms are estimated with

$$
\frac{1}{\epsilon^{2}} \int_{\mathbb{R}^{d}}\left|\overline{\mathrm{A}}_{0}\left[\partial^{\alpha}, \overline{\mathrm{A}}_{0}^{-1} \overline{\mathrm{L}}\right] \pi \widetilde{\mathrm{w}}\right|^{2} \mathrm{dx} \leq \frac{\mathrm{c}_{0}}{\epsilon^{2}}\left|\overline{\mathrm{A}}_{0}\right|_{\infty}^{2}\left|\partial_{\times}\left(\overline{\mathrm{A}}_{0}^{-1} \overline{\mathrm{L}}\right)\right|_{l-2}^{2}|\pi \widetilde{\mathrm{w}}|_{l-2}^{2} \leq \frac{\mathrm{c}_{2}}{\epsilon^{2}}|\pi \widetilde{\mathrm{w}}|_{l-2}^{2},
$$

and collecting all contributions we have established that

$$
\sum_{0 \leq|\alpha| \leq l-1} \frac{|\alpha| !}{\alpha !}\left(\left|\mathrm{f}^{\alpha}\right|_{0}^{2}+\epsilon_{d}^{2}\left|\mathrm{~g}^{\alpha}\right|_{0}^{2}\right) \leq \mathrm{c}_{2}\left(|\mathrm{f}|_{l-1}^{2}+\epsilon_{d}^{2}\left|\mathrm{~g}_{\mathrm{II}}\right|_{l-1}^{2}+|\delta \widetilde{\mathrm{w}}|_{l-1}^{2}+\epsilon_{d}^{2}\left|\delta \widetilde{\mathrm{w}}_{\mathrm{II}}\right|_{l}^{2}\right)+\frac{\mathrm{c}_{2}}{\epsilon^{2}}|\pi \widetilde{\mathrm{w}}|_{l-2}^{2}
$$

In order to control the last term $\left(\mathrm{c}_{2} / \epsilon^{2}\right)|\pi \widetilde{\mathrm{w}}|_{l-2}^{2}$ we now have to write the $(l-1)$ th projected equation. Multiplying equation (A.6) by $\frac{1}{\epsilon} \partial^{\alpha} \pi \widetilde{w}$ and $|\alpha| ! / \alpha$ !, integrating over $\mathbb{R}^{d}$, and summing over $0 \leq|\alpha| \leq l-1$, yields that

$$
\begin{aligned}
\frac{1}{\epsilon} \partial_{t} E_{l-1}^{2}(\pi \widetilde{\mathrm{w}})+\frac{\delta_{1}}{\epsilon^{2}}|\pi \widetilde{\mathrm{w}}|_{l-1}^{2} \leq \mathrm{c}_{1}|\delta \widetilde{\mathrm{w}}|_{l}^{2}+\mathrm{c}_{1} \epsilon_{d}^{2}\left|\delta \widetilde{\mathrm{w}}_{\mathrm{II}}\right|_{l+1}^{2} & +\frac{\mathrm{c}_{1}}{\epsilon}\left|\partial_{t} \mathrm{w}\right|_{l-1} E_{l-1}^{2}(\pi \widetilde{\mathrm{w}}) \\
& +\sum_{0 \leq|\alpha| \leq l-1} \mathrm{c}_{1} \frac{|\alpha| !}{\alpha !}\left(\left|\mathrm{f}^{\alpha}\right|_{0}^{2}+\epsilon_{d}^{2}\left|\mathrm{~g}^{\alpha}\right|_{0}^{2}\right)
\end{aligned}
$$


Using the previous estimates of $\left|\mathrm{f}^{\alpha}\right|_{0}$ and $\left|\mathrm{g}^{\alpha}\right|_{0}$, the inequality $|\phi|_{l-2}^{2} \leq \beta|\phi|_{l-1}^{2}+C(\beta)|\phi|_{0}^{2}$, the equivalence of $\widehat{E}_{l-1}^{2}(\pi \widetilde{\mathrm{w}})$ and $E_{l-1}^{2}(\pi \widetilde{\mathrm{w}})$, and combining the derived $l$ th estimate with the projected $l$ th estimate, we now obtain that

$$
\begin{aligned}
& \delta_{1}\left|\partial_{t} \widetilde{\mathrm{w}}\right|_{l-1}^{2}+\frac{1}{\epsilon} \partial_{t}\left(\widehat{E}_{l-1}^{2}(\pi \widetilde{\mathrm{w}})+E_{l-1}^{2}(\pi \widetilde{\mathrm{w}})\right)+\frac{\delta_{1}}{\epsilon^{2}}|\pi \widetilde{\mathrm{w}}|_{l-1}^{2} \leq \mathrm{c}_{1}|\delta \widetilde{\mathrm{w}}|_{l}^{2}+\mathrm{c}_{1} \epsilon_{d}^{2}\left|\delta \widetilde{\mathrm{w}}_{\mathrm{II}}\right|_{l+1}^{2} \\
& \quad+\frac{\mathrm{c}_{1}}{\epsilon}\left|\partial_{t} \mathrm{w}\right|_{l-1} \widehat{E}_{l-1}^{2}(\pi \widetilde{\mathrm{w}})+\mathrm{c}_{2}|\mathrm{f}|_{l-1}^{2}+\mathrm{c}_{2} \epsilon_{d}^{2}\left|\mathrm{~g}_{\mathrm{II}}\right|_{l-1}^{2}+\mathrm{c}_{2}|\delta \widetilde{\mathrm{w}}|_{l-1}^{2}+\mathrm{c}_{2} \epsilon_{d}^{2}\left|\delta \widetilde{\mathrm{w}}_{\mathrm{II}}\right|_{l}^{2}+\frac{\mathrm{c}_{2}}{\epsilon^{2}}|\pi \widetilde{\mathrm{w}}|_{0}^{2} .
\end{aligned}
$$

We now combine this inequality to the $l$ th order governing equation (A.9) multiplied by a large constant $\mathrm{K}_{1}$ only depending on $\mathcal{O}_{1}$ so as to compensate the term $\mathrm{c}_{1} \epsilon_{d}^{2}|\delta \widetilde{\mathrm{w}}|_{l+1}^{2}$ in the right hand side. We also add the zeroth order derived equation (A.4) multiplied by a factor $\mathrm{K}_{2}$ in order to compensate for the term $\left(\mathrm{c}_{2} / \epsilon^{2}\right)|\pi \widetilde{\mathrm{w}}|_{0}^{2}$. We have then obtained a governing inequality in the form

$$
\begin{gathered}
\partial_{t}\left(\frac{1}{\epsilon} \widehat{E}_{l-1}^{2}(\pi \widetilde{\mathrm{w}})+\frac{1}{\epsilon} E_{l-1}^{2}(\pi \widetilde{\mathrm{w}})+\mathrm{K}_{1} E_{l}^{2}(\delta \widetilde{\mathrm{w}})+\frac{\mathrm{K}_{2}}{\epsilon} E_{0}^{2}(\pi \widetilde{\mathrm{w}})\right)+\delta_{1}\left|\partial_{t} \widetilde{\mathrm{w}}\right|_{l-1}^{2}+\frac{\delta_{1}}{\epsilon^{2}}|\pi \widetilde{\mathrm{w}}|_{l-1}^{2} \\
+\epsilon_{d} \delta_{1}\left|\delta \widetilde{\mathrm{w}}_{\mathrm{II}}\right|_{l+1}^{2}+\frac{\delta_{1}}{\epsilon}|\pi \widetilde{\mathrm{w}}|_{l}^{2} \leq \frac{\mathrm{c}_{2}}{\epsilon}\left(1+\left|\partial_{t} \mathrm{w}\right|_{l-1}\right) \widehat{E}_{l-1}^{2}(\pi \widetilde{\mathrm{w}})+\mathrm{c}_{2}|\mathrm{f}|_{l-1}^{2} \\
+\mathrm{c}_{2} \epsilon_{d}\left|\mathrm{~g}_{\mathrm{II}}\right|_{l-1}^{2}+\mathrm{c}_{2}\left(1+\left|\partial_{t} \mathrm{w}\right|_{l-1}\right) E_{l}^{2}(\delta \widetilde{\mathrm{w}})+\mathrm{c}_{2}|\mathrm{f}|_{l} E_{l}(\delta \widetilde{\mathrm{w}}) .
\end{gathered}
$$

From the Gronwall inequality we get after some algebra the second estimate (3.15).

Finally, the various occurencies of the constant $c_{2}$ in the proof all involve simple polynomials in $M$ with positive coefficients, either arising as simple multiplication by $M$ or through the estimate (A.3) so that the final constant $\mathrm{c}_{2}$ is an increasing function of $M$ and the proof is complete.

\section{B Time derivatives estimates}

We present in Appendix B the proof of the extra time derivative estimates of Theorem 3.4. Since $l \geq l_{0}+4$ we first deduce from the governing equations and the regularity of the solution $\mathrm{w}$ that

$$
\begin{aligned}
& \partial_{t} \mathrm{w}_{\mathrm{I}} \in \mathcal{C}^{0}\left([0, \bar{\tau}], H^{l-1}\right) \cap \mathcal{C}^{1}\left([0, \bar{\tau}], H^{l-3}\right), \\
& \partial_{t} \mathrm{w}_{\mathrm{II}} \in \mathcal{C}^{0}\left([0, \bar{\tau}], H^{l-2}\right) \cap \mathcal{C}^{1}\left([0, \bar{\tau}], H^{l-4}\right) \cap L^{2}\left((0, \bar{\tau}), H^{l-1}\right),
\end{aligned}
$$

although we do not have uniform bounds in these spaces because of the stiff sources. Letting $\widehat{\mathrm{M}}_{i j}(\mathrm{w})=$ $\overline{\mathrm{M}}_{i j}+\partial_{\mathrm{w}} \overline{\mathrm{B}}_{i j}, i, j \in \mathcal{D}$, the third order tensors $\widehat{\mathrm{M}}_{i j}$ have at least regularity $\varkappa-3$ and the governing equations may then be written

$$
\overline{\mathrm{A}}_{0}(\mathrm{w}) \partial_{t} \mathrm{w}+\sum_{i \in \mathcal{D}} \overline{\mathrm{A}}_{i}(\mathrm{w}) \partial_{i} \mathrm{w}-\epsilon_{d} \sum_{i, j \in \mathcal{D}} \overline{\mathrm{B}}_{i j}(\mathrm{w}) \partial_{i} \partial_{j} \mathrm{w}+\frac{1}{\epsilon} \overline{\mathrm{L}}(\mathrm{w}) \mathrm{w}=\epsilon_{d} \sum_{i, j \in \mathcal{D}} \widehat{\mathrm{M}}_{i j}(\mathrm{w}) \partial_{i} \mathrm{w} \partial_{j} \mathrm{w} .
$$

Differenciating this system with respect to time we obtain that $\widetilde{\mathrm{w}}=\partial_{t} \mathrm{w}$ satisfies

$$
\overline{\mathrm{A}}_{0}(\mathrm{w}) \partial_{t} \widetilde{\mathrm{w}}+\sum_{i \in \mathcal{D}} \overline{\mathrm{A}}_{i}(\mathrm{w}) \partial_{i} \widetilde{\mathrm{w}}-\epsilon_{d} \sum_{i, j \in \mathcal{D}} \overline{\mathrm{B}}_{i j}(\mathrm{w}) \partial_{i} \partial_{j} \widetilde{\mathrm{w}}+\frac{1}{\epsilon} \overline{\mathrm{L}}(\mathrm{w}) \widetilde{\mathrm{w}}=\mathrm{f}_{t}+\epsilon_{d} \mathrm{~g}_{t}
$$

where

$$
\begin{gathered}
\mathrm{f}_{t}=-\sum_{i \in \mathcal{D}} \overline{\mathrm{A}}_{0} \partial_{\mathrm{w}}\left(\overline{\mathrm{A}}_{0}^{-1} \overline{\mathrm{A}}_{i}\right) \widetilde{\mathrm{w}} \partial_{i} \mathrm{w}-\frac{1}{\epsilon} \overline{\mathrm{A}}_{0} \partial_{\mathrm{w}}\left(\overline{\mathrm{A}}_{0}^{-1} \overline{\mathrm{L}}\right) \widetilde{\mathrm{w}} \pi \mathrm{w} \\
\mathrm{g}_{t}=\sum_{i, j \in \mathcal{D}} \overline{\mathrm{A}}_{0} \partial_{\mathrm{w}}\left(\overline{\mathrm{A}}_{0}^{-1} \overline{\mathrm{B}}_{i j}\right) \widetilde{\mathrm{w}} \partial_{i} \partial_{j} \mathrm{w}+\sum_{i, j \in \mathcal{D}} \overline{\mathrm{A}}_{0} \partial_{\mathrm{w}}\left(\overline{\mathrm{A}}_{0}^{-1} \widehat{\mathrm{M}}_{i j}\right) \widetilde{\mathrm{w}} \partial_{i} \mathrm{w} \partial_{j} \mathrm{w} \\
+\sum_{i, j \in \mathcal{D}} \widehat{\mathrm{M}}_{i j} \partial_{i} \widetilde{\mathrm{w}} \partial_{j} \mathrm{w}+\sum_{i, j \in \mathcal{D}} \widehat{\mathrm{M}}_{i j} \partial_{i} \mathrm{w} \partial_{j} \widetilde{\mathrm{w}} .
\end{gathered}
$$


Step 0. The expressions (B.2)(B.3) are not convenient and are rewritten by using the generalized inverse $\overline{\mathrm{L}}^{\sharp}$ of $\overline{\mathrm{L}}$ such that $\overline{\mathrm{L}} \overline{\mathrm{L}}^{\sharp}=\overline{\mathrm{L}}^{\sharp} \overline{\mathrm{L}}=\pi, \overline{\mathrm{L}}^{\sharp}=\left(\overline{\mathrm{L}}^{\sharp}\right), N\left(\overline{\mathrm{L}}^{\sharp}\right)=\mathcal{E}$ and $R\left(\overline{\mathrm{L}}^{\sharp}\right)=\mathcal{E}^{\perp}$. This pseudo inverse $\overline{\mathrm{L}}^{\sharp}$ is the generalized inverse of $\overline{\mathrm{L}}$ with prescribed range $R\left(\overline{\mathrm{L}}^{\sharp}\right)=\mathcal{E}^{\perp}$ and nullspace $N\left(\overline{\mathrm{L}}^{\sharp}\right)=\mathcal{E}$, and is a smooth function of $\bar{L}[14,25]$. Using $\bar{L}^{\sharp}$ we deduce from the governing equations that

$$
\frac{\pi \mathrm{w}}{\epsilon}=-\overline{\mathrm{L}}^{\sharp}\left(\overline{\mathrm{A}}_{0}(\mathrm{w}) \pi \widetilde{\mathrm{w}}+\sum_{i \in \mathcal{D}} \overline{\mathrm{A}}_{i}(\mathrm{w}) \partial_{i} \mathrm{w}-\epsilon_{d} \sum_{i, j \in \mathcal{D}} \overline{\mathrm{B}}_{i j}(\mathrm{w}) \partial_{i} \partial_{j} \mathrm{w}-\epsilon_{d} \overline{\mathrm{b}}\right),
$$

keeping in mind that $\overline{\mathrm{L}}^{\sharp}=\overline{\mathrm{L}}^{\sharp} \pi$ and $\pi \overline{\mathrm{A}}_{0}=\overline{\mathrm{A}}_{0} \pi$. We may thus rewrite $\mathrm{f}_{t}$ and $\mathrm{g}_{t}$ in the form

$$
\begin{aligned}
& \mathrm{f}_{t}=-\sum_{i \in \mathcal{D}} \overline{\mathrm{A}}_{0} \partial_{\mathrm{w}}\left(\overline{\mathrm{A}}_{0}^{-1} \overline{\mathrm{A}}_{i}\right) \widetilde{\mathrm{w}} \partial_{i} \mathrm{w}+\sum_{i \in \mathcal{D}} \overline{\mathrm{A}}_{0} \partial_{\mathrm{w}}\left(\overline{\mathrm{A}}_{0}^{-1} \overline{\mathrm{L}}\right) \widetilde{\mathrm{w}} \overline{\mathrm{L}}^{\sharp} \overline{\mathrm{A}}_{i} \partial_{i} \mathrm{w}+\overline{\mathrm{A}}_{0} \partial_{\mathrm{w}}\left(\overline{\mathrm{A}}_{0}^{-1} \overline{\mathrm{L}}\right) \widetilde{\mathrm{w}} \overline{\mathrm{L}}^{\sharp} \overline{\mathrm{A}}_{0} \pi \widetilde{\mathrm{w}}, \\
& \mathrm{g}_{t}=\sum_{i, j \in \mathcal{D}} \overline{\mathrm{A}}_{0} \partial_{\mathrm{w}}\left(\overline{\mathrm{A}}_{0}^{-1} \overline{\mathrm{B}}_{i j}\right) \widetilde{\mathrm{w}} \partial_{i} \partial_{j} \mathrm{w}+\sum_{i, j \in \mathcal{D}} \overline{\mathrm{A}}_{0} \partial_{\mathrm{w}}\left(\overline{\mathrm{A}}_{0}^{-1} \widehat{\mathrm{M}}_{i j}\right) \widetilde{\mathrm{w}} \partial_{i} \mathrm{w} \partial_{j} \mathrm{w} \\
&-\sum_{i, j \in \mathcal{D}} \overline{\mathrm{A}}_{0} \partial_{\mathrm{w}}\left(\overline{\mathrm{A}}_{0}^{-1} \overline{\mathrm{L}}\right) \widetilde{\mathrm{w}} \bar{L}^{\sharp} \overline{\mathrm{B}}_{i j} \partial_{i} \partial_{j} \mathrm{w}-\sum_{i, j \in \mathcal{D}} \overline{\mathrm{A}}_{0} \partial_{\mathrm{w}}\left(\overline{\mathrm{A}}_{0}^{-1} \overline{\mathrm{L}}\right) \widetilde{\mathrm{w}} \bar{L}^{\sharp} \overline{\mathrm{M}}_{i j} \partial_{i} \mathrm{w} \partial_{j} \mathrm{w} \\
&+\sum_{i, j \in \mathcal{D}} \widehat{\mathrm{M}}_{i j} \partial_{i} \widetilde{\mathrm{w}} \partial_{j} \mathrm{w}+\sum_{i, j \in \mathcal{D}} \widehat{\mathrm{M}}_{i j} \partial_{i} \mathrm{w} \partial_{j} \widetilde{\mathrm{w}} .
\end{aligned}
$$

The equation (B.1) is thus formally 'linearized' except for the quadratic term $\overline{\mathrm{A}}_{0} \partial_{\mathrm{w}}\left(\overline{\mathrm{A}}_{0}^{-1} \overline{\mathrm{L}}\right) \widetilde{\mathrm{w}} \overline{\mathrm{L}}^{\sharp} \overline{\mathrm{A}}_{0} \pi \widetilde{\mathrm{w}}$ in $\mathrm{f}_{t}$ arising from the stiff sources. In order to derive the new estimates, we use a similar notation as in Theorem 3.1 and Theorem 3.3. In particular, $\delta_{1}=\delta\left(\mathcal{O}_{1}\right) \leq 1$ denotes a generic small constant only depending on $\mathcal{O}_{1}, c_{1}=c_{1}\left(\mathcal{O}_{1}\right) \geq 1$ a generic large constant only depending on $\mathcal{O}_{1}$, and $\mathrm{c}_{2}=\mathrm{c}_{2}\left(\mathcal{O}_{1}, b\right) \geq$ 1 a generic large constant depending on $\mathcal{O}_{1}$ and the constant $b$ of Theorem 3.3.

Step 1. Zeroth order entropic estimates for $\widetilde{w}=\partial_{t} w$. We multiply (B.1) by $\widetilde{w}$ and proceed as in the proof of Theorem 3.1. Using the symmetry of $\overline{\mathrm{A}}_{0}$ and $\overline{\mathrm{A}}_{i}$, integrating over $\mathbb{R}^{d}$, and using the Garding inequality, we obtain that

$$
\partial_{t} E_{0}^{2}(\widetilde{\mathrm{w}})+\epsilon_{d} \delta_{1}\left|\widetilde{\mathrm{w}}_{\mathrm{II}}\right|_{1}^{2}+\frac{\delta_{1}}{\epsilon}|\pi \widetilde{\mathrm{w}}|_{0}^{2} \leq \mathrm{c}_{2}\left(1+\left|\partial_{t} \mathrm{w}\right|_{l_{0}}\right) E_{0}^{2}(\widetilde{\mathrm{w}})+\int_{\mathbb{R}^{d}}\left\langle\mathrm{f}_{t}+\epsilon_{d} \mathrm{~g}_{t}, \widetilde{\mathrm{w}}\right\rangle \mathrm{dx} .
$$

All terms linear in $\widetilde{w}$ in $f_{t}$ and $g_{t}$ yield contributions majorized by $c_{1}\left|\partial_{x} w\right|_{l_{0}}|\widetilde{w}|_{0}^{2}+\epsilon_{d} c_{1}\left(\left|\partial_{x} w\right|_{l_{0}}^{2}+\right.$ $\left.\left|\partial_{x}^{2} \mathrm{w}_{\text {II }}\right| l_{0}\right)|\widetilde{\mathrm{w}}|_{0}^{2}+\epsilon_{d} \mathrm{c}_{1}\left|\partial_{x} \mathrm{w}\right|_{l_{0}}\left|\widetilde{\mathrm{w}}_{\mathrm{II}}\right|_{1}|\widetilde{\mathrm{w}}|_{0}$ and the quadratic term is also majorized by $c_{1}|\widetilde{\mathrm{w}}| l_{0}|\widetilde{\mathrm{w}}|_{0}^{2}$. Using the estimates of Theorem 3.3 and $l_{0}=[d / 2]+1>d$, we obtain after some algebra

$$
\partial_{t} E_{0}^{2}(\widetilde{\mathrm{w}})+\epsilon_{d} \delta_{1}\left|\widetilde{\mathrm{w}}_{\mathrm{II}}\right|_{1}^{2}+\frac{\delta_{1}}{\epsilon}|\pi \widetilde{\mathrm{w}}|_{0}^{2} \leq \mathrm{c}_{2}\left(1+\left|\partial_{t} \mathrm{w}\right|_{l_{0}}\right) E_{0}^{2}(\widetilde{\mathrm{w}}) .
$$

From the Gronwall inequality and the estimates (3.18) we thus obtain that for $0 \leq t \leq \bar{\tau}$

$$
\sup _{0 \leq \tau \leq t}|\widetilde{\mathrm{w}}(\tau)|_{0}^{2}+\epsilon_{d} \int_{0}^{t}\left|\widetilde{\mathrm{w}}_{\mathrm{II}}(\tau)\right|_{1}^{2} d \tau+\frac{1}{\epsilon} \int_{0}^{t}|\pi \widetilde{\mathrm{w}}|_{0}^{2} d \tau \leq \mathrm{c}_{1}^{2} \exp \left(\mathrm{c}_{2}\left(t+M_{1} \sqrt{t}\right)\right)\left|\widetilde{\mathrm{w}}_{0}\right|_{0}^{2}
$$

Step 2. The projected zeroth order estimate. We multiply the governing equation (B.1) by $(1 / \epsilon) \pi \widetilde{w}$ and proceed as in the proof of Theorem 3.1. Integrating over $\mathbb{R}^{d}$ we obtain that

$$
\begin{aligned}
\frac{1}{2 \epsilon} \partial_{t} E_{0}^{2}(\pi \widetilde{\mathrm{w}})+\frac{\delta_{1}}{\epsilon^{2}}|\pi \widetilde{\mathrm{w}}|_{0}^{2} \leq \frac{\mathrm{c}_{1}}{\epsilon}\left|\partial_{t} \mathrm{w}\right|_{l_{0}}|\pi \widetilde{\mathrm{w}}|_{0}^{2}+\frac{\mathrm{c}_{1}}{\epsilon}|\widetilde{\mathrm{w}}|_{1}|\pi \widetilde{\mathrm{w}}|_{0} \\
+\epsilon_{d} \frac{\mathrm{c}_{1}}{\epsilon}\left|\widetilde{\mathrm{w}}_{\mathrm{II}}\right|_{2}|\pi \widetilde{\mathrm{w}}|_{0}+\frac{1}{\epsilon} \int_{\mathbb{R}^{d}}\left\langle\mathrm{f}_{t}+\epsilon_{d} \mathrm{~g}_{t}, \pi \widetilde{\mathrm{w}}\right\rangle \mathrm{dx} .
\end{aligned}
$$


All terms linear in $\widetilde{w}$ in $f_{t}$ and $g_{t}$ yield contributions majorized by

$$
\frac{\mathrm{c}_{1}}{\epsilon}\left|\partial_{\times} \mathrm{w}\right| l_{0}|\widetilde{\mathrm{w}}|_{0}|\pi \widetilde{\mathrm{w}}|_{0}+\epsilon_{d} \frac{\mathrm{c}_{1}}{\epsilon}\left(\left|\partial_{\mathrm{x}} \mathrm{w}\right|_{l_{0}}^{2}+\left|\partial_{\times}^{2} \mathrm{w}_{\mathrm{II}}\right| l_{0}\right)|\widetilde{\mathrm{w}}|_{0}|\pi \widetilde{\mathrm{w}}|_{0}+\left.\epsilon_{d} \frac{\mathrm{c}_{1}}{\epsilon}\left|\partial_{\times} \mathrm{w}\right|_{l_{0}}\left|\widetilde{\mathrm{w}}_{\mathrm{II}}\right|\right|_{1}|\pi \widetilde{\mathrm{w}}|_{0},
$$

and the quadratic term is also majorized by $\left(c_{1} / \epsilon\right)|\widetilde{w}|_{l_{0}}|\pi \widetilde{w}|_{0}^{2}$. Using the Cauchy-Schwarz inequality and the estimates (3.18) of Theorem 3.3 we obtain after some algebra

$$
\frac{1}{\epsilon} \partial_{t} E_{0}^{2}(\pi \widetilde{\mathrm{w}})+\frac{\delta_{1}}{\epsilon^{2}}|\pi \widetilde{\mathrm{w}}|_{0}^{2} \leq \frac{\mathrm{c}_{1}}{\epsilon}\left|\partial_{t} \mathrm{w}\right|_{l_{0}} E_{0}^{2}(\pi \widetilde{\mathrm{w}})+\mathrm{c}_{2}|\widetilde{\mathrm{w}}|_{1}^{2}+\epsilon_{d}^{2} \mathrm{c}_{1}\left|\widetilde{\mathrm{w}}_{\mathrm{II}}\right|_{2}^{2}
$$

Step 3. The $l^{\prime}$ th zeroth order estimate. We let for short $l^{\prime}=l-2$ so that $l^{\prime} \geq l_{0}+2$. Differentiating the hyperbolic-parabolic system (B.1) with respect to the space variable and denoting by $\partial^{\alpha}$ the $\alpha$ th derivative spatial operator we obtain that

$$
\overline{\mathrm{A}}_{0}(\mathrm{w}) \partial_{t} \partial^{\alpha} \widetilde{\mathrm{w}}+\sum_{i \in \mathcal{D}} \overline{\mathrm{A}}_{i}(\mathrm{w}) \partial_{i} \partial^{\alpha} \widetilde{\mathrm{w}}-\epsilon_{d} \sum_{i, j \in \mathcal{D}} \overline{\mathrm{B}}_{i j}(\mathrm{w}) \partial_{i} \partial_{j} \partial^{\alpha} \widetilde{\mathrm{w}}+\frac{1}{\epsilon} \overline{\mathrm{L}}(\mathrm{w}) \partial^{\alpha} \widetilde{\mathrm{w}}=\mathrm{f}_{t}^{\alpha}+\epsilon_{d} \mathbf{g}_{t}^{\alpha},
$$

with

$$
\begin{gathered}
\mathrm{f}_{t}^{\alpha}=\overline{\mathrm{A}}_{0} \partial^{\alpha}\left(\overline{\mathrm{A}}_{0}^{-1} \mathrm{f}_{t}\right)-\sum_{i \in \mathcal{D}} \overline{\mathrm{A}}_{0}\left[\partial^{\alpha}, \overline{\mathrm{A}}_{0}^{-1} \overline{\mathrm{A}}_{i}\right] \partial_{i} \widetilde{\mathrm{w}}-\frac{1}{\epsilon} \overline{\mathrm{A}}_{0}\left[\partial^{\alpha}, \overline{\mathrm{A}}_{0}^{-1} \overline{\mathrm{L}}\right] \pi \widetilde{\mathrm{w}}, \\
\mathrm{g}_{t}^{\alpha}=\overline{\mathrm{A}}_{0} \partial^{\alpha}\left(\overline{\mathrm{A}}_{0}^{-1} \mathrm{~g}_{t}\right)+\sum_{i, j \in \mathcal{D}} \overline{\mathrm{A}}_{0}\left[\partial^{\alpha}, \overline{\mathrm{A}}_{0}^{-1} \overline{\mathrm{B}}_{i j}\right] \partial_{i} \partial_{j} \widetilde{\mathrm{w}} .
\end{gathered}
$$

Multiplying equation (B.8) by $\partial^{\alpha} \widetilde{\mathrm{w}}|\alpha| ! / \alpha$ !, integrating over $\mathbb{R}^{d}$, summing over $0 \leq|\alpha| \leq l^{\prime}$, and proceeding as for the zeroth order estimate, we obtain that

$$
\begin{array}{r}
\partial_{t} E_{l^{\prime}}^{2}(\widetilde{\mathrm{w}})+\epsilon_{d} \delta_{1}\left|\widetilde{\mathrm{w}}_{\mathrm{II}}\right|_{l^{\prime}+1}^{2} \\
+\frac{\delta_{1}}{\epsilon}|\pi \widetilde{\mathrm{w}}|_{l^{\prime}}^{2} \leq \mathrm{c}_{2}\left(1+\left|\partial_{t} \mathrm{w}\right|_{l_{0}}\right) E_{l^{\prime}}^{2}(\widetilde{\mathrm{w}}) \\
+\sum_{0 \leq|\alpha| \leq l^{\prime}} \frac{|\alpha| !}{\alpha !} \int_{\mathbb{R}^{d}}\left\langle\mathrm{f}_{t}^{\alpha}+\epsilon_{d} \mathrm{~g}_{t}^{\alpha}, \partial^{\alpha} \widetilde{\mathrm{w}}\right\rangle \mathrm{d} \mathrm{x},
\end{array}
$$

and we have to investigate the residuals associated with $\mathrm{f}_{t}^{\alpha}$ and $\mathrm{g}_{t}^{\alpha}$. Keeping in mind that the zeroth order terms with $|\alpha|=0$ have already been examined in Step 1, we only have to analyze the terms such that $1 \leq|\alpha| \leq l^{\prime}$.

The terms in $\mathrm{f}_{t}^{\alpha}$ and $\mathrm{g}_{t}^{\alpha}$ arising from $\overline{\mathrm{A}}_{0} \partial^{\alpha}\left(\overline{\mathrm{A}}_{0}^{-1} \mathrm{f}_{t}\right)$ and $\overline{\mathrm{A}}_{0} \partial^{\alpha}\left(\overline{\mathrm{A}}_{0}^{-1} \mathrm{~g}_{t}\right)$ yield contributions majorized by $c_{2}\left(|\widetilde{\mathrm{w}}|_{l^{\prime}}^{2}+\epsilon_{d}|\widetilde{\mathrm{w}}|_{l^{\prime}}\left|\widetilde{\mathrm{w}}_{\mathrm{II}}\right| l_{l^{\prime}+1}+|\widetilde{\mathrm{w}}|_{l^{\prime}}^{3}\right)$, where the last term arise from the quadratic contribution in $\mathrm{f}_{t}$. On the other hand, the commutators contributions are estimated as

$$
\begin{gathered}
\left|\int_{\mathbb{R}^{d}}\left\langle\overline{\mathrm{A}}_{0}\left[\partial^{\alpha}, \overline{\mathrm{A}}_{0}^{-1} \overline{\mathrm{A}}_{i}\right] \partial_{i} \widetilde{\mathrm{w}}, \partial^{\alpha} \widetilde{\mathrm{w}}\right\rangle \mathrm{dx}\right| \leq \mathrm{c}_{2}|\mathrm{w}| l_{l^{\prime}}|\widetilde{\mathrm{w}}|_{l^{\prime}}^{2}, \\
\frac{1}{\epsilon}\left|\int_{\mathbb{R}^{d}}\left\langle\overline{\mathrm{A}}_{0}\left[\partial^{\alpha}, \overline{\mathrm{A}}_{0}^{-1} \overline{\mathrm{L}}\right] \pi \widetilde{\mathrm{w}}, \partial^{\alpha} \widetilde{\mathrm{w}}\right\rangle \mathrm{dx}\right| \leq \frac{\mathrm{c}_{2}}{\epsilon}|\mathrm{w}|_{l^{\prime}}|\pi \widetilde{\mathrm{w}}|_{l^{\prime}-1}|\pi \widetilde{\mathrm{w}}|_{l^{\prime}}, \\
\left|\int_{\mathbb{R}^{d}}\left\langle\overline{\mathrm{A}}_{0}\left[\partial^{\alpha}, \overline{\mathrm{A}}_{0}^{-1} \overline{\mathrm{B}}_{i j}\right] \partial_{i} \partial_{j} \widetilde{\mathrm{w}}, \partial^{\alpha} \widetilde{\mathrm{w}}\right\rangle \mathrm{dx}\right| \leq \mathrm{c}_{2}|\mathrm{w}| l_{l^{\prime}}\left|\widetilde{\mathrm{w}}_{\mathrm{II}}\right|_{l^{\prime}+1}\left|\widetilde{\mathrm{w}}_{\mathrm{II}}\right|_{l^{\prime}} .
\end{gathered}
$$

Collecting all contributions and after some algebra we have established that

$$
\partial_{t} E_{l^{\prime}}^{2}(\widetilde{\mathrm{w}})+\epsilon_{d} \delta_{1}\left|\widetilde{\mathrm{w}}_{\mathrm{II}}\right|_{l^{\prime}}^{2}+\frac{\delta_{1}}{\epsilon}|\pi \widetilde{\mathrm{w}}|_{l^{\prime}}^{2} \leq \mathrm{c}_{2}\left(1+\left|\partial_{t} \mathrm{w}\right|_{l^{\prime}-1}\right) E_{l^{\prime}}^{2}(\widetilde{\mathrm{w}})+\frac{\mathrm{c}_{2}}{\epsilon}|\pi \widetilde{\mathrm{w}}|_{l^{\prime}-1}^{2}
$$

In order to handle the term $\left(c_{2} / \epsilon\right)|\pi \widetilde{\mathrm{w}}|_{l-1}^{2}$ in the right hand side arising from the commutators we use the inequality $|\phi|_{l^{\prime}-1}^{2} \leq \beta|\phi|_{l^{\prime}}^{2}+C(\beta)|\phi|_{0}^{2}$ and add the resulting inequality to the projected zeroth order inequality (B.7) obtained in Step 2. Assuming that $l^{\prime} \geq l_{0}+1 \geq 2$ we obtain that

$$
\partial_{t}\left(E_{l^{\prime}}^{2}(\widetilde{\mathrm{w}})+\frac{1}{\epsilon} E_{0}^{2}(\pi \widetilde{\mathrm{w}})\right)+\epsilon_{d} \delta_{1}\left|\widetilde{\mathrm{W}}_{\mathrm{II}}\right|_{l^{\prime}+1}^{2}+\frac{\delta_{1}}{\epsilon}|\pi \widetilde{\mathrm{w}}|_{l^{\prime}}^{2}
$$




$$
+\frac{\delta_{1}}{\epsilon^{2}}|\pi \widetilde{\mathrm{w}}|_{0}^{2} \leq \mathrm{c}_{2}\left(1+\left|\partial_{t} \mathrm{w}\right|_{l^{\prime}-1}\right)\left(E_{l^{\prime}}^{2}(\widetilde{\mathrm{w}})+\frac{1}{\epsilon} E_{0}^{2}(\pi \widetilde{\mathrm{w}})\right),
$$

From the Gronwall inequality, we obtain that

$$
\begin{gathered}
\sup _{0 \leq \tau \leq t}\left(E_{l^{\prime}}^{2}(\widetilde{\mathrm{w}}(\tau))+\frac{1}{\epsilon} E_{0}^{2}(\pi \widetilde{\mathrm{w}}(\tau))\right)+\epsilon_{d} \int_{0}^{t}\left|\widetilde{\mathrm{w}}_{\mathrm{II}}(\tau)\right|_{l^{\prime}+1}^{2} d \tau+\frac{1}{\epsilon} \int_{0}^{t}|\pi \widetilde{\mathrm{w}}|_{l^{\prime}}^{2} d \tau+\frac{1}{\epsilon^{2}} \int_{0}^{t}|\pi \widetilde{\mathrm{w}}|_{0}^{2} d \tau \\
\leq \mathrm{c}_{1}^{2} \exp \left(\mathrm{c}_{2}\left(t+M_{1} \sqrt{t}\right)\right)\left(\left|\widetilde{\mathrm{w}}_{0}\right|_{l^{\prime}}^{2}+\frac{1}{\epsilon}\left|\pi \widetilde{\mathrm{w}}_{0}\right|_{0}^{2}\right),
\end{gathered}
$$

which is the $l^{\prime}$ order estimate for $\widetilde{\mathrm{w}}=\partial_{t} \mathrm{w}$ and $l^{\prime}=l-2$.

Step 4. Application of linearized estimates to $\widetilde{w}=\partial_{t} w$. Taking into account the $l^{\prime}$ order estimate for $\widetilde{w}=\partial_{t} \mathrm{w}$, it is now possible to apply the linearized estimates (3.15). From the expression (B.5) and (B.6) of $\mathrm{f}_{t}$ and $\mathrm{g}_{t}$, we indeed obtain that $\mathrm{f}_{t} \in \mathcal{C}^{0}\left([0, \bar{\tau}], H^{l-2}\right)$ and $\mathrm{g}_{t} \in \mathcal{C}^{0}\left([0, \bar{\tau}], H^{l-3}\right)$ so that $\mathrm{f}_{t} \in \mathcal{C}^{0}\left([0, \bar{\tau}], H^{l-3}\right) \cap L^{1}\left((0, \bar{\tau}), H^{l-2}\right)$ and $\mathrm{g}_{t} \in \mathcal{C}^{0}\left([0, \bar{\tau}], H^{l-3}\right)$ with uniform estimates in terms of

$$
\left|\mathrm{w}_{0}-\mathrm{w}^{\star}\right|_{l}^{2}+\frac{1}{\epsilon}\left|\pi \mathrm{w}_{0}\right|_{l-1}^{2}+\left|\widetilde{\mathrm{w}}_{0}\right|_{l-2}^{2}+\frac{1}{\epsilon}\left|\pi \widetilde{\mathrm{w}}_{0}\right|_{l-3}^{2} .
$$

The estimates (3.37) are then a direct consequence of (3.14) and (3.15) applied with $l^{\prime}=l-2$ in place of $l$ and with $\widetilde{\mathrm{w}}=\partial_{t} \mathrm{w}$.

\section{References}

[1] G. Billet, V. Giovangigli, and G. de Gassowski, Impact of Volume Viscosity on a Shock/Hydrogen Bubble Interaction, Comb. Theory Mod., 12 (2008), pp. 221-248.

[2] D. Bresch and B. Desjardins, On the Existence of Global Weak Solutions to the Navier-Stokes Equations for Viscous Compressible and Heat Conducting Fluids. J. Math. Pure Appl., 87 (2007), pp. $57-90$.

[3] D. Bruno and V. Giovangigli, Relaxation of Internal Temperature and Volume Viscosity, Phys. Fluids, 23 (2011), 093104.

[4] D. Bruno and V. Giovangigli, Erratum: "Relaxation of internal temperature and volume viscosity", Phys. Fluids, 25 (2013), 039902.

[5] D. Bruno, F. Esposito, and V. Giovangigli, Relaxation of Rotational-Vibrational Energy and Volume Viscosity in H-H2 Mixtures, J. Chem. Physics, 138 (2013), 084302.

[6] S. Chapman and T.G. Cowling, The Mathematical Theory of Non-Uniform Gases, Cambridge University Press, (1970).

[7] G. Q. Chen, C. D. Levermore, and T. P. Liu, Hyperbolic conservation laws with stiff relaxation terms and entropy, Comm. Pure Appl. Math., 47 (1994), pp. 787-830.

[8] C. Dafermos, Hyperbolic Conservation Laws in Continuum Physics, Springer, Heidelberg, (2000).

[9] C. M. Dafermos, Hyperbolic Systems of Balance Laws with Weak Dissipation II, J. Hyp. Diff. Eq., 10 (2013), pp. 173-179.

[10] C. M. Dafermos, BV Solutions for hyperbolic systems of balance laws with relaxation, J. Diff. Eqs., 255 (2013), pp. 2521-2533.

[11] G. Emanuel, Bulk viscosity of a dilute polyatomic gas, Phys. Fluids, A, 2 (1990), pp. 2252-2254.

[12] A. Ern and V. Giovangigli, Multicomponent Transport Algorithms, Lecture Notes in Physics, New series Monographs m24, (1994). 
[13] A. Ern and V. Giovangigli, Volume viscosity of dilute polyatomic gas mixtures, Eur. J. Mech., B/Fluids, 14 (1995), pp. 653-669.

[14] A. Ern and V. Giovangigli, Projected iterative algorithms with application to multicomponent transport, Linear Algebra Appl., 250 (1997), pp. 289-315.

[15] E. Feireisl, Dynamics of viscous compressible fluids. Oxford, 2004.

[16] J.H. Ferziger, H.G. Kaper, Mathematical theory of transport processes in gases, North Holland, Amsterdam, (1972).

[17] K. O. Friedrichs and P. D. Lax, Systems of Conservation laws with a Convex Extension, Proc. Nat. Acad. Sci. USA 68 (1971) pp. 1686-1688.

[18] V. Giovangigli, Multicomponent flow modeling, Birkhaüser, Boston, (1999).

[19] V. Giovangigli and B. Graille, Asymptotic Stability of Equilibrium States for Ambipolar Plasmas, Math. Mod. Meth. Appl. Sci., 14, (2004), pp. 1361-1399.

[20] V. Giovangigli and M. Massot, Asymptotic Stability of Equilibrium States for Multicomponent Reactive Flows. Math. Mod. Meth. App. Sci., 8 (1998), pp. 251-297.

[21] V. Giovangigli and M. Massot, Entropic Structure of Multicomponent Reactive Flows with Partial Equilibrium Reduced Chemistry, Math. Meth. Appl. Sci., 27 (2004), pp. 739-768.

[22] V. Giovangigli and L. Matuszewski, Supercritical Fluid Thermodynamics from Equations of State, Phys. D, 241 (2012), pp. 649-670.

[23] V. Giovangigli and L. Matuszewski, Mathematical modeling of supercritical multicomponent reactive fluids, Math. Mod. Meth. App. Sci., 23 (2013), pp. 2193-2251.

[24] V. Giovangigli and B. Tran, Mathematical Analysis of a Saint-Venant Model with Variable Temperature, Math. Mod. Meth. Appl. Sci., 20 (2010), pp. 1-47.

[25] V. Giovangigli and W.-A. Yong, Volume viscosity and fast internal energy relaxation: Symmetrization and Chapman-Enskog expansion, Kin. Rel. Models, (accepted for publication).

[26] S. Godunov, An interesting class of quasilinear systems, Sov. Math. Dokl, 2 (1961), pp. 947-949.

[27] R. E. Graves and B. Argrow, Bulk viscosity : past to present, Journal of Thermophysics and Heat Transfer, 13 (1999), pp. 337-342.

[28] B. Hanouzet and R. Natalini, Global existence of smooth solutions for partially dissipative hyperbolic systems with a convex entropy, Arch. Rational Mech. Anal., 169 (2003), pp. 89-117.

[29] S. Kawashima, Systems of a Hyperbolic-Parabolic Composite Type, with applications to the equations of Magnetohydrodynamics Doctoral Thesis, Kyoto University (1984).

[30] S. Kawashima, Large-time Behavior of Solutions to Hyperbolic-Parabolic Systems of Conservations Laws and Applications. Proc. Roy. Soc. Edinburgh, 106A (1987) 169-1944.

[31] S. Kawashima and Y. Shizuta, On the Normal Form of the Symmetric Hyperbolic-Parabolic Systems Associated with the Conservation Laws. Tôhoku Math. J, 40 (1988), pp. 449-464.

[32] S. Kawashima and W.-A. Yong, Dissipative structure and entropy for hyperbolic systems of conservation laws. Arch. Rat. Mech. Anal., 174 (2004), pp. 345-364.

[33] A. Majda, Compresible fluid flow and systems of conservation laws in several space variables, Springer, New York, (1984).

[34] A. Matsumura and T. Nishida, The Initial Value Problem for the Equations of Motion of a Viscous and Heat-Conductive Fluids, J. Math. Kyoto Univ., 200, (1980), pp. 67-104. 
[35] F.R. McCourt, J.J. Beenakker, W.E. Köhler, I. Kuscer, Non equilibrium phenomena in polyatomic gases, Volume I: Dilute gases, Volume II: Cross sections, scattering and rarefied gases, Clarendon Press, Oxford, (1990).

[36] E. Nagnibeda and E. Kustova, Non-equilibrium reacting gas flow, Springer Verlag, Berlin, (2009).

[37] R. Natalini, Convergence to equilibrium for the relaxation approximation of conservation laws, Commun. Pure Appl. Math., 49 (1996), pp. 795-823.

[38] G.J. Prangsma, A.H. Alberga, and J.J.M. Beenakker, Ultrasonic determination of the volume viscosity of $\mathrm{N}_{2}, \mathrm{CO}, \mathrm{CH}_{4}$, and $\mathrm{CD}_{4}$ between 77 and 300K, Physica, 64 (1973), pp. 278-288.

[39] D. Serre, Systèmes de Lois de Conservation I et II, Diderot Editeur, Art et Science, Paris, 1996.

[40] D. Serre, The Structure of Dissipative Viscous System of Conservation laws, Physica D, 239 (2010), pp. 1381-1386.

[41] L. Tisza, Supersonic absorption and Stokes viscosity relation, Physical Review, 61 (1941), pp. $531-536$.

[42] A.I. Vol'pert and S.I. Hudjaev, On the Cauchy Problem for Composite Systems of Nonlinear Differential Equations, Math USSR Sbornik, 16 (1972) pp. 517-544.

[43] W.-A. Yong, Singular perturbations of first-order hyperbolic systems with stiff source terms, J. Diff. Equ., 155 (1999), pp. 89-132.

[44] W.-A. Yong, Entropy and global existence for hyperbolic balance laws, Arch. Rat. Mech. Anal., 172 (2004), pp. 247-266.

[45] W.-A. Yong, An interesting class of partial differential equations, J. Math. Physics, 49 (2008), 033503.

[46] W.-A. Yong and K. Zumbrun, Existence of relaxation shock profiles for hyperbolic conservation laws, Siam J. Appl. Math., 60 (2000), pp. 1665-1675. 